\title{
Gamma-ray burst optical light-curve zoo: comparison with X-ray observations ${ }^{\star \star \star \star}$
}

\author{
E. Zaninoni ${ }^{1,2}$, M. G. Bernardini ${ }^{1}$, R. Margutti ${ }^{3}$, S. Oates ${ }^{4}$, and G. Chincarini ${ }^{1,5}$
}

\author{
1 INAF - Osservatorio Astronomico di Brera, via Bianchi 46, 23807 Merate, Italy \\ e-mail: elena.zaninoni@brera.inaf.it \\ 2 University of Padova, Physics \& Astronomy Dept. Galileo Galilei, vicolo dell’'Osservatorio, 35131 Padova, Italy \\ ${ }^{3}$ Harvard-Smithsonian Center for Astrophysics, 60 Garden Street, Cambridge, MA 02138, USA \\ ${ }^{4}$ Mullard Space Science Laboratory, University College London, Holmbury St. Mary, Dorking, Surrey RH5 6NT, UK \\ 5 Univerisità Milano Bicocca, Dip. Fisica G. Occhialini, P.zza della Scienza 3, 20126 Milano, Italy
}

Received 1 February 2013 / Accepted 13 March 2013

\begin{abstract}
Aims. We present a comprehensive analysis of the optical and X-ray light curves (LCs) and spectral energy distributions (SEDs) of a large sample of gamma-ray burst (GRB) afterglows to investigate the relationship between the optical and X-ray emission after the prompt phase. We consider all data available in the literature, which where obtained with different instruments.

Methods. We collected the optical data from the literature and determined the shapes of the optical LCs. Then, using previously presented X-ray data, we modeled the optical/X-ray SEDs. We studied the SED parameter distributions and compared the optical and $\mathrm{X}$-ray LC slopes and shapes.

Results. The optical and X-ray spectra become softer as a function of time while the gas-to-dust ratios of GRBs are higher than the values calculated for the Milky Way and the Large and Small Magellanic Clouds. For 20\% of the GRBs the difference between the optical and X-ray slopes is consistent with 0 or $1 / 4$ within the uncertainties (we did it not consider the steep decay phase), while in the remaining $80 \%$ the optical and X-ray afterglows show significantly different temporal behaviors. Interestingly, we find an indication that the onset of the forward shock in the optical LCs (initial peaks or shallow phases) could be linked to the presence of the X-ray flares. Indeed, when X-ray flares are present during the steep decay, the optical LC initial peak or end plateau occurs during the steep decay; if instead the X-ray flares are absent or occur during the plateau, the optical initial peak or end plateau takes place during the X-ray plateau.

Conclusions. The forward-shock model cannot explain all features of the optical (e.g. bumps, late re-brightenings) and X-ray (e.g. flares) LCs. However, the synchrotron model is a viable mechanism for GRBs at late times. In particular, we found a relationship between the presence of the X-ray flares and the shape of the optical LC that indicates a link between the prompt emission and the optical afterglow.
\end{abstract}

Key words. gamma-ray burst: general - radiation mechanisms: non-thermal

\section{Introduction}

Gamma-ray bursts (GRBs) are the most powerful sources of electromagnetic radiation in the Universe, with an isotropic luminosity that can reach values of $10^{54} \mathrm{erg} \mathrm{s}^{-1}$. The Swift satellite (Gehrels et al. 2004), launched in November 2004, opened a new era for the study and understanding of GRB phenomena; thanks to the rapid response of the instruments with a small field of view, it was discovered that both the X-ray light-curve (LC; e.g. Nousek et al. 2006; Zhang et al. 2006) and the optical LC (e.g. Roming et al. 2009) have a complex shape. The rapid computation of the GRB position by the Swift Burst Alert Telescope (BAT; Barthelmy et al. 2005), refined with an accuracy of few arcseconds by the Swift X-ray Telescope (XRT, Burrows et al. 2005), and the instantaneous dissemination to the community

\footnotetext{
* Appendix $\mathrm{C}$ is available in electronic form at http: //www . aanda.org

$\star \star$ Full Tables C.1-C.6 are only available at the CDS via anonymous ftp to cdsarc.u-strasbg. fr $(130.79 .128 .5)$ or via http://cdsarc.u-strasbg.fr/viz-bin/qcat?J/A+A/557/A12
}

via the $\mathrm{GCN}^{1}$ allows a growing number of robotic telescopes to promptly repoint to the source. Some examples are the Robotic Optical Transient Search Experiment (ROTSE-III, Akerlof et al. 2003), the Rapid Eye Mount telescope (REM, Zerbi et al. 2001; Chincarini et al. 2003), the Gamma-Ray Burst Optical/NearInfrared detector (GROND, Greiner et al. 2008), Liverpool (LT) and Faulkes telescopes (Gomboc et al. 2006), Télescopes à Action Rapide pour les Objets Transitoires (TAROT, Klotz et al. 2008b), and others.

Some generic features have been previously found in optical LCs. Optical and X-ray LCs are different at early times in the majority of cases (Melandri et al. 2008b; Rykoff et al. 2009; Oates et al. 2009, 2011). In particular, Oates et al. $(2009,2011)$ noted that the optical LCs can decay or rise before $500 \mathrm{~s}$ after the trigger in the observer frame and do not show the steep decay as the X-ray LCs; after $2000 \mathrm{~s}$ the optical and X-ray LCs have similar slopes. Panaitescu \& Vestrand $(2008,2011)$ divided the optical LCs according to their initial behavior (peaky or shallow). Peaks were associated to impulsive ejecta releases, while

1 Gamma-ray Coordinates Network (GCN), http://gcn.gsfc. nasa.gov/gcn3_archive.html 
plateau phases were assumed to belong to the energy released by a long-lived central engine. Chromatic and achromatic breaks have been found in the optical and X-ray LCs (Melandri et al. 2008b; Rykoff et al. 2009; Oates et al. 2009, 2011; Panaitescu \& Vestrand 2011). Moreover, the brighter optical LCs decay faster (Oates et al. 2009, 2011, 2012). When optical and X-ray LCs do not share the same temporal decay, X-ray LCs have been found to decay faster (Oates et al. 2009, 2011; Panaitescu \& Vestrand 2011). For only a few GRBs with shallow X-ray decay phases we find a corresponding shallow decay in the optical (Rykoff et al. 2009; Li et al. 2012). Flares can occasionally appear in optical LCs and likely linked to the long term central engine activity (Li et al. 2012).

Previous works mainly concentrated on data obtained by a single telescope (e.g. Melandri et al. 2008b; Klotz et al. 2009a; Cenko et al. 2009; Oates et al. 2009, 2011; Rykoff et al. 2009) and only a few authors compared the data from different instruments (e.g. Nysewander et al. 2009a; Kann et al. 2010b, 2011; Li et al. 2012; Liang et al. 2012). For example, Kann et al. (2010b, 2011) focused on the classification of the optical LCs and the host galaxy extinction. Li et al. (2012) and Liang et al. (2012) concentrated on the optical LC shapes and particular features, as bumps, plateaus, late rebrightenings. Other works studied the dust extinction of the GRB host galaxies (e.g. Schady et al. 2012, 2010; Zafar et al. 2011) or the circumburst density profiles around GRB progenitors (Schulze et al. 2011).

In this paper we analyze a large sample of $68 \mathrm{GRBs}$ with optical and X-ray observations and known redshift, detected between December 2004 and December 2010. Our starting sample includes 165 GRBs with known redshift presented by Margutti et al. 2013 (hereafter M 13). We collected the optical data from the literature and obtained well-sampled optical LCs for 68 GRBs from different telescopes and instruments. To compare the optical and X-ray observations, we used the X-ray data extracted and analyzed in M 13. We focused on the relationship between the optical and X-ray emission, comparing their restframe temporal and spectroscopic properties and their energetics. In particular, we investigated the forward-shock model and the synchrotron emission in the GRB afterglow. In Sect. 2 we detail the sample selection criteria, the data selection and reduction, the procedure followed for fitting the optical LCs and of the spectral energy distributions (SEDs). The results of our analysis are presented in Sect. 3 and are discussed in Sect. 4. The main conclusions are drawn in Sect. 5. We adopt standard values of the cosmological parameters: $H_{0}=70 \mathrm{~km} \mathrm{~s}^{-1} \mathrm{Mpc}^{-1}, \Omega_{\mathrm{M}}=0.27$, and $\Omega_{\Lambda}=0.73$. For the temporal and spectral energy index, $\alpha$ and $\beta$, we used the convention $F_{v}(t, v) \propto t^{-\alpha} v^{-\beta}$. Errors are given at $1 \sigma$ confidence level unless otherwise stated.

\section{Sample selection and data analysis}

We considered the 165 GRBs with known and secure redshift ${ }^{2}$ observed by Swift/XRT between December 2004 and December 2010, presented in M 13. Among these GRBs, we selected those with optical observations and with optical data available in the literature. We used only the data from refereed papers and with more than five data points per filter. In this way we obtained a subsample of 68 long GRBs (Table C.9). This criterion automatically excluded short GRBs. The selection in spectroscopic

\footnotetext{
2 From Margutti et al. (2013) we used only optical spectroscopic redshifts and photometric redshifts for which we are able to exclude sources of degeneracy. We list the redshifts and luminosity distances of the GRBs of our sample in table5c.dat at CDS.
}

redshift from the optical afterglow introduces a bias against highly absorbed optical afterglows (Fynbo et al. 2009; Perley et al. 2009a; Greiner et al. 2011). In fact, GRBs with optical spectroscopy have a substantially lower X-ray excess absorption and a substantially smaller fraction of dark bursts (Fynbo et al. 2009). On the other hand, our final aim is to compare $\mathrm{X}$-ray and optical rest frame properties, and this can be carried out only with bright and well-sampled optical LCs. Within these constraints we collected a large number of data from more than one hundred telescopes with different instruments and filters (Table C.9). We analyzed the energetics and luminosities of these GRBs and calculated the SED in the optical/X-ray frequency range.

\subsection{Optical data}

Magnitudes were converted into flux densities following standard practice (see Appendix A for details). For this analysis, we used only LCs that had more than five data points per filter and excluded upper limits. This is the best compromise between statistics (in the sense that we do not discard too many GRBs) and reliability (robust fit and energy measurement). All collected data will be available online ${ }^{3}$.

For each filter we fitted the optical LCs with the same fit functions as for the X-ray data in M 13. We chose these functional forms because they represent the optical LC shapes well and it facilitates comparing optical with X-ray data. We used optical data not corrected for reddening and these fit functions:

\section{Single power-law:}

$$
F_{v}(t)=N t^{-\alpha} .
$$

2. One or more smoothed broken power-laws:

$$
F_{v}(t)=\sum_{\mathrm{i}} N_{i}\left(\left(\frac{t}{t_{\mathrm{b}, i}}\right)^{-\frac{\alpha_{1, i}}{s_{i}}}+\left(\frac{t}{t_{\mathrm{b}, i}}\right)^{-\frac{\alpha_{2, i}}{s_{i}}}\right)^{s_{i}} .
$$

3. Sum of power-law and smoothed broken power-law:

$$
F_{v}(t)=N_{1} t^{-\alpha_{1}}+N_{2}\left(\left(\frac{t}{t_{\mathrm{b}}}\right)^{-\frac{\alpha_{2}}{s}}+\left(\frac{t}{t_{\mathrm{b}}}\right)^{-\frac{\alpha_{3}}{s}}\right)^{s},
$$

where $\alpha$ is the power-law decay index, $t_{\mathrm{b}}$ the break time, $s$ the smoothness parameter (always fixed to $-0.3,-0.5$ or -0.8 ) and $N$ the normalization. The best-fit parameters were determined using the IDL Levenberg-Marquard least-squares fit routine (MPFIT) supplied by Markwardt $(2009)^{4}$. The best-fitting function was chosen considering the $\chi^{2}$ statistics. The bestfitting parameters are reported in Table C. $1^{5}$. The best-fit of the optical LCs and their residuals are shown in Figs. C.1-C.9.

\subsection{X-ray data}

The X-ray spectra were extracted using the method presented in M 13 (see also references therein). We fitted them with Xspec and the function tbabs*ztbabs*pow, which considers the hydrogen column density absorption of the Milky Way ( $\left.N_{\mathrm{H}, \mathrm{MW}}\right)$ and of the host galaxy $\left(N_{\mathrm{H}, \text { host }}\right)$. The $N_{\mathrm{H}, \mathrm{MW}}$ was calculated with

\footnotetext{
3 The data will be available on the web site http://www. elenazaninoni.com

4 http://www.physics.wisc.edu/ craigm/idl/fitting.html 5 The complete and machine-readable form of the table is provided at CDS (table1c.dat).
} 
the nh tool, which uses the weighted average value from the Kalberla et al. (2005) map. The output data obtained from the X-ray spectrum are $N_{\mathrm{H}, \mathrm{X}}$ and the X-ray photon index ${ }^{6}\left(\Gamma_{\mathrm{X}}\right)$ (Table C. $4^{7}$ ).

\subsection{Optical/X-ray SEDs}

For each GRB, we created optical/X-ray SEDs at one or more epochs (Table C.8). The time intervals for the SEDs were chosen taking into account the shape of the X-ray and optical shapes: a) they belong to a determined phase of the X-ray LC that is steep decay, plateau or normal decay to avoid the X-ray LC breaks. In this way we obtained a SED both at early times (where the afterglow emission could be influenced by the prompt emission) and at late times (where the afterglow emission is very unlikely to be contaminated by the prompt emission); b) sometimes the SEDs were constructed during X-ray and optical flares. For the optical data, we did not extrapolate the optical LC, so that if for a given filter no data were available, the filter was excluded from the SED. For each filter with data in this time range, we calculated the flux density by integrating the optical LC over the considered time interval.

We fitted the optical/X-ray SED accounting for absorption in the optical and X-ray ranges both locally (i.e., in the GRB host galaxy) and arising from the Milky Way (MW). For the optical band we used the extinction laws given by Pei (1992) (Eq. (20) and Table 4 therein) for the MW and the Large Magellanic Cloud (LMC) and Small Magellanic Cloud (SMC). For the $\mathrm{X}$-ray data, we considered the model for the photoelectric cross section per HI-atom units for a given metallicity presented by Morrison \& McCammon (1983), assuming solar metallicity.

We first considered the case that the X-ray and the optical bands lie in the same spectral segment, hence the SED-fitting function is a combination of the absorption laws presented above and a single power-law:

$f_{v}(v)=f_{0} v_{\mathrm{obs}}^{-\beta_{\mathrm{op}, \mathrm{X}}}$,

where $v_{\mathrm{obs}}$ is the observed frequency, $\beta_{\mathrm{op}, \mathrm{X}}$ the spectral index ${ }^{6}$ and $f_{0}$ the normalization. From the input parameters, the Galactic hydrogen column density $\left(N_{\mathrm{H}, \mathrm{MW}}\right)$, the Galactic reddening $\left(E(B-V)_{\mathrm{MW}}{ }^{8}\right)$ and the redshift $(z)$, we obtained the host galaxy hydrogen column density $N_{\mathrm{H}, \mathrm{op}, \mathrm{X}}$, reddening $E(B-V)_{\text {host }}$, and the spectral index $\beta_{\mathrm{op}, \mathrm{X}}$.

Then, we examined the hypothesis that the cooling frequency is between the optical and the X-ray bands and fit the data using the absorption laws plus a broken-power law:

$$
\begin{aligned}
& f_{v}(v)= F_{0}\left(v_{\mathrm{obs}}^{-\beta_{\mathrm{op}}} \operatorname{step}\left(v_{\mathrm{obs}, \mathrm{BR}}-v_{\mathrm{obs}}\right)\right. \\
&+v_{\mathrm{obs}}^{-\beta_{\mathrm{X}}} \gamma_{\mathrm{obs}, \mathrm{BR}}-\beta_{\mathrm{op}} \\
&\left.\operatorname{ste} p\left(v_{\mathrm{obs}}-v_{\mathrm{obs}, \mathrm{BR}}\right)\right),
\end{aligned}
$$

where step is the step function, $v_{\mathrm{obs}, \mathrm{BR}}$ the observer frame break frequency between the optical and X-ray band, $\beta_{\mathrm{op}}$ the optical spectral index, and $\beta_{\mathrm{X}}$ the $\mathrm{X}$-ray spectral index. The fit was performed in two ways: with $\beta_{\text {op }}$ let free to vary or fixed as $\beta_{\mathrm{op}}=\beta_{\mathrm{X}}-0.5$, as predicted theorically by Sari et al. (1998) and empirically by Zafar et al. (2011). Letting $\beta_{\text {op }}$ free to vary

\footnotetext{
6 The spectral index $\beta$ is related to the photon index $\Gamma$ by $\Gamma=\beta+1$.

7 The complete and machine-readable form of the table is provided at CDS (table4c.dat).

8 The $E(B-V)$ values were taken from NASA/IPAC Extragalactic Database (NED) website (http://ned.ipac.caltech.edu/forms/ calculator.html), which uses the Schlegel et al. (1998) maps.
}

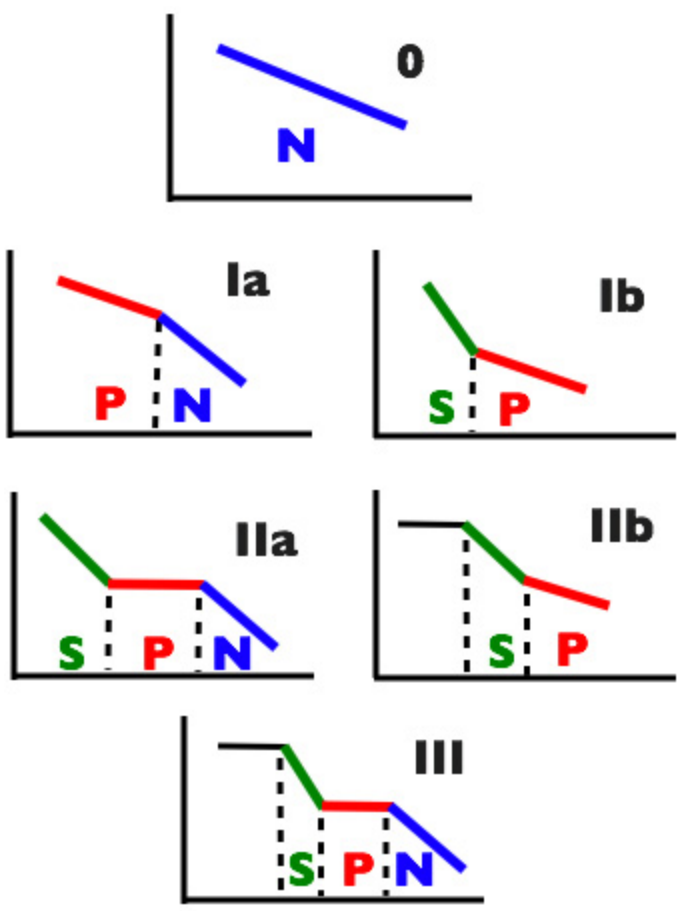

Fig. 1. Cartoon representing the X-ray LCs types. For the X-ray LC shapes we used the code presented in M13. Following the prescription of Bernardini et al. (2012a) and M 13, we denoted the different parts of the LCs as a) steep decay ( $S$, green): first segment of type $I b$ and $I I a$ LCs; the second segment of type $I I b$ and $I I I \mathrm{LCs} ; b)$ plateau $(P$, red): the first segment of type $I a$ LCs; the second segment of type $I b$ and $I I a$ LCs; the third segment of type $I I b$ and $I I I$ LCs; $c$ ) normal decay $(N$, blue): type $0 \mathrm{LCs}$; the second segment of type Ia LCs; the third segment of type IIa LCs; the forth segment of type III LCs.

did not lead to reliable results. Therefore the best-fit functions of the optical/X-ray SEDs may either be a single power-law or a broken power-law with $\beta_{\text {op }}=\beta_{\mathrm{X}}-0.5$. To determine if a broken or a single power-law was required, we used an F-test probability $<5 \%$ as threshold. The results of this selection are presented in Table C.8 and Figs. C.10-C.18. The fit parameters are listed in Table C. $2^{9}$. In Table C. $6^{10}$ we list the optical data used for the SEDs.

\section{Results}

\subsection{Spectral parameter distributions}

We considered the parameters obtained from fitting the optical/X-ray SEDs $\left(\beta, N_{\mathrm{H}}, E(B-V), \nu_{\mathrm{BR}}\right)$ with a single or broken power-law, as selected in Sect. 2.3 (Table C.8), and with a p-value ${ }^{11}$ higher than 0.05 . We eliminated the results with errors larger than the data themselves and set to zero negative data that where consistent with 0 within the uncertainties. In total, $78 \%$ of our fits have p-value $>0.05$ and 33 GRBs have more than one SED with a p-value $>0.05$. For the steep-decay SEDs, we

9 The complete and machine-readable forms of the tables are provided at CDS (table2c.dat, table3c.dat).

10 The complete and machine-readable form of the table is provided at CDS (table6c.dat).

11 The p-value is a number between zero and one and it is the probability of obtaining a test statistic at least as extreme as the one that was actually observed, assuming that the null hypothesis is true. 
Table 1. Characteristic quantities describing the parameter distributions (number of elements (\#), mean $(m)$, median $(M)$, standard deviation (SD)), and best-fitting values from a Gaussian fit (mean $(\mu)$, standard deviation $(\sigma))$.

\begin{tabular}{|c|c|c|c|c|c|c|}
\hline Name & $\#$ & $\mu$ & $\sigma$ & $m$ & SD & $M$ \\
\hline$\beta_{\mathrm{op}, \mathrm{X}}$ & 65 & $0.95 \pm 0.01$ & $0.24 \pm 0.02$ & 1.03 & 0.50 & 0.95 \\
\hline$\beta_{\mathrm{X}}$ & 34 & $0.97 \pm 0.02$ & $0.18 \pm 0.02$ & 0.99 & 0.22 & 0.95 \\
\hline$\beta_{\mathrm{op}}$ & 34 & $0.47 \pm 0.02$ & $0.19 \pm 0.02$ & 0.48 & 0.20 & 0.48 \\
\hline $\log \left(N_{\mathrm{H}, \mathrm{BR}} / \mathrm{cm}^{-2}\right)$ & 28 & $21.6 \pm 0.11$ & $0.51 \pm 0.11$ & 21.7 & 0.57 & 21.7 \\
\hline $\log \left(N_{\mathrm{H}, \mathrm{PL}} / \mathrm{cm}^{-2}\right)$ & 43 & $21.7 \pm 0.06$ & $0.41 \pm 0.06$ & 21.9 & 0.48 & 21.7 \\
\hline$E(B-V)_{\mathrm{MW}}$ & 30 & $0.19 \pm 0.02$ & $0.14 \pm 0.02$ & 11.1 & 59.1 & 0.22 \\
\hline$E(B-V)_{\mathrm{LMC}}$ & 14 & - & - & 0.20 & 0.16 & 0.18 \\
\hline$E(B-V)_{\mathrm{SMC}}$ & 25 & - & - & 0.20 & 0.29 & 0.10 \\
\hline$A_{\mathrm{V}, \mathrm{MW}}$ & 30 & $0.56 \pm 0.10$ & $0.42 \pm 0.11$ & 34.2 & 182. & 0.69 \\
\hline$A_{\mathrm{V}, \mathrm{LMC}}$ & 14 & - & - & 0.63 & 0.51 & 0.57 \\
\hline$A_{\mathrm{V}, \mathrm{SMC}}$ & 25 & - & - & 0.59 & 0.87 & 0.29 \\
\hline $\log \left(v_{\text {rest }, \mathrm{BR}} / \mathrm{Hz}\right)$ & 28 & - & - & 16.20 & 0.98 & 16.1 \\
\hline $\log \left[\left(N_{\mathrm{H}} / \mathrm{cm}^{-2}\right) /\left(A_{\mathrm{V}} / \mathrm{mag}\right)\right]_{\mathrm{MW}}$ & 20 & $21.9 \pm 0.05$ & $0.04 \pm 0.05$ & 22.0 & 0.56 & 21.8 \\
\hline $\log \left[\left(N_{\mathrm{H}} / \mathrm{cm}^{-2}\right) /\left(A_{\mathrm{V}} / \mathrm{mag}\right)\right]_{\mathrm{LMC}}$ & 12 & $22.6 \pm 0.08$ & $0.32 \pm 0.11$ & 22.4 & 0.95 & 22.6 \\
\hline $\log \left[\left(N_{\mathrm{H}} / \mathrm{cm}^{-2}\right) /\left(A_{\mathrm{V}} / \mathrm{mag}\right)\right]_{\mathrm{SMC}}$ & 20 & $21.8 \pm 0.16$ & $0.87 \pm 0.18$ & 22.4 & 2.05 & 22.2 \\
\hline$\beta_{\mathrm{onX}}^{\mathrm{S}}$ & 9 & - & - & 0.85 & 0.22 & 0.85 \\
\hline$\beta_{\mathrm{op}, \mathrm{X}}^{\mathrm{P}, \mathrm{L}}$ & 24 & $0.92 \pm 0.03$ & $0.21 \pm 0.04$ & 0.95 & 0.27 & 0.95 \\
\hline$\beta_{\mathrm{op}, \mathrm{X}}^{\mathrm{N}, \Lambda}$ & 31 & $0.99 \pm 0.03$ & $0.23 \pm 0.03$ & 2.35 & 1.08 & 2.35 \\
\hline$\beta_{\mathrm{X}}^{\mathrm{P}, X}$ & 13 & $0.96 \pm 0.02$ & $0.10 \pm 0.02$ & 0.85 & 0.22 & 0.85 \\
\hline$\beta_{\mathrm{X}}^{\mathrm{N}}$ & 19 & $1.00 \pm 0.08$ & $0.29 \pm 0.09$ & 1.15 & 0.27 & 1.15 \\
\hline$\beta_{\mathrm{op}}^{\mathrm{P}}$ & 13 & $0.49 \pm 0.04$ & $0.14 \pm 0.04$ & 0.35 & 0.22 & 0.35 \\
\hline$\beta_{\mathrm{op}}^{\mathrm{N}}$ & 19 & $0.47 \pm 0.08$ & $0.29 \pm 0.09$ & 0.65 & 0.27 & 0.65 \\
\hline $\log \left(N_{\mathrm{H}, \mathrm{PL}}^{\mathrm{S}} / \mathrm{cm}^{-2}\right)$ & 6 & - & - & 22.2 & 0.63 & 22.2 \\
\hline $\log \left(N_{\mathrm{H}, \mathrm{PL}}^{\mathrm{P}} / \mathrm{cm}^{-2}\right)$ & 17 & $22.00 \pm 0.14$ & $0.67 \pm 0.23$ & 22.0 & 0.52 & 22.2 \\
\hline $\log \left(N_{\mathrm{H}, \mathrm{PL}}^{\mathrm{N}} / \mathrm{cm}^{-2}\right)$ & 19 & $21.70 \pm 0.04$ & $0.30 \pm 0.04$ & 22.2 & 0.86 & 22.2 \\
\hline $\log \left(N_{\mathrm{H}, \mathrm{BR}}^{\mathrm{P}} / \mathrm{cm}^{-2}\right)$ & 10 & $21.00 \pm 1.20$ & $0.93 \pm 0.870$ & 21.8 & 0.632 & 21.8 \\
\hline $\log \left(N_{\mathrm{H}, \mathrm{BR}}^{\mathrm{N}} / \mathrm{cm}^{-2}\right)$ & 17 & $21.90 \pm 0.05$ & $0.23 \pm 0.04$ & 21.8 & 0.86 & 21.8 \\
\hline $\log \left(v_{\text {rest.,BR }}^{\mathrm{P}} / \mathrm{Hz}\right)$ & 14 & - & - & 16.80 & 1.08 & 16.80 \\
\hline $\log \left(v_{\text {rest } \mathrm{BR}}^{\mathrm{N}} / \mathrm{Hz}\right)$ & 16 & - & - & 16.20 & 1.08 & 16.20 \\
\hline $\log \left(L_{\mathrm{R}, 500 \mathrm{~s}} /\left(\mathrm{erg} \mathrm{s}^{-1}\right)\right)$ & 64 & $45.90 \pm 0.06$ & $0.83 \pm 0.06$ & 46.00 & 3.72 & 45.90 \\
\hline $\log \left(L_{\mathrm{R}, 1 \mathrm{~h}} /\left(\mathrm{erg} \mathrm{s}^{-1}\right)\right)$ & 57 & $45.40 \pm 0.06$ & $0.73 \pm 0.06$ & 45.90 & 4.77 & 45.40 \\
\hline $\log \left(L_{\mathrm{R}, 11 \mathrm{~h}} /\left(\mathrm{erg} \mathrm{s}^{-1}\right)\right)$ & 40 & $44.50 \pm 0.08$ & $0.74 \pm 0.08$ & 45.70 & 7.44 & 44.50 \\
\hline $\log \left(L_{\mathrm{R}, 1 \text { day }} /\left(\mathrm{erg} \mathrm{s}^{-1}\right)\right)$ & 32 & $44.20 \pm 0.11$ & $0.83 \pm 0.13$ & 44.20 & 0.68 & 44.20 \\
\hline $\log \left(F_{\mathrm{R}, 920-1200 \mathrm{~s}} /\left(\mathrm{erg} \mathrm{cm}^{-2} \mathrm{~s}^{-1}\right)\right)$ & 46 & $-11.41 \pm 0.07$ & $0.34 \pm 0.07$ & -11.31 & 0.80 & -11.37 \\
\hline $\log \left(F_{1 \mathrm{keV}, 920-1200 \mathrm{~s}} /\left(\mathrm{erg} \mathrm{cm}^{-2} \mathrm{~s}^{-1}\right)\right)$ & 52 & $-12.54 \pm 0.06$ & $0.49 \pm 0.06$ & -12.40 & 0.72 & -12.45 \\
\hline
\end{tabular}

Notes. The subscript PL indicates the values obtained by fitting the SED with a single power-law, while BR indicates a broken power-law. S stands for steep decay, $\mathrm{P}$ for plateau, and $\mathrm{N}$ for normal decay. The values of $E(B-V)$ depend on the extinction model law used to fit the SED: Milky Way (MW), Large Magellanic Cloud (LMC) and Small Magellanic Cloud (SMC).

obtained a good fit (p-value $>0.05$ ) with a single power-law for $9 / 13$ SEDs and with a broken power-law for $1 / 13$. We where unable to fit three SEDs. Accordingly, we did not consider parameters obtained with a broken power-law for the steep decay (i.e. $\left.v_{\mathrm{rest}, \mathrm{BR}}, \beta_{\mathrm{op}}, \beta_{\mathrm{X}}, N_{\mathrm{H}, \mathrm{BR}}\right)$ in the distribution.

For every distribution of the best-fitting values, we calculated the mean $(m)$, the standard deviation (SD), and the median $(M)$. When possible, we fitted the distributions with a Gaussian function obtaining the mean $(\mu)$ and the standard deviation $(\sigma)$. All results are listed in Table 1. In Fig. 2 (top panels) we show the parameter distributions differentiating between the data obtained by fitting the SEDs with a single-power law (red, PL) or a broken-power law (blue, BR) and in Fig. 3 the distributions distiguishing the SED parameters extracted during the X-ray steepdecay phase (blue, S), the plateau (red, P) or the normal decay phase (gray, N) (see Fig. 1). We defined the X-ray LCs shapes as in M 13 (Fig. 1): 0 if there are no breaks, Ia or Ib if there is a break, IIa or IIb if there are two breaks, and III if there are three breaks. The differentiation between model Ia and Ib depends on the smoothness parameter $s<0$ and $s>0$. Type IIa is the canonical shape (e.g. Nousek et al. 2006; Zhang et al. 2006), while type IIb starts with a shallow phase followed by a steep decay and then a normal decay.

We present in the bottom left panel of Fig. 2 the distributions of host $E(B-V)$ for the MW, SMC and LMC for the SEDs for which we were able to differentiate between the extinction laws used.

\subsubsection{Spectral index}

The mean spectral slope computed by fitting a single powerlaw is $\mu\left(\beta_{\mathrm{op}, \mathrm{X}}\right)=0.95 \pm 0.01$. This value is consistent with the spectral slope $\mu\left(\beta_{\mathrm{X}}\right)=0.97 \pm 0.02$ obtained using a broken 
E. Zaninoni et al.: The gamma-ray burst optical light-curve zoo: comparison with the X-ray observations
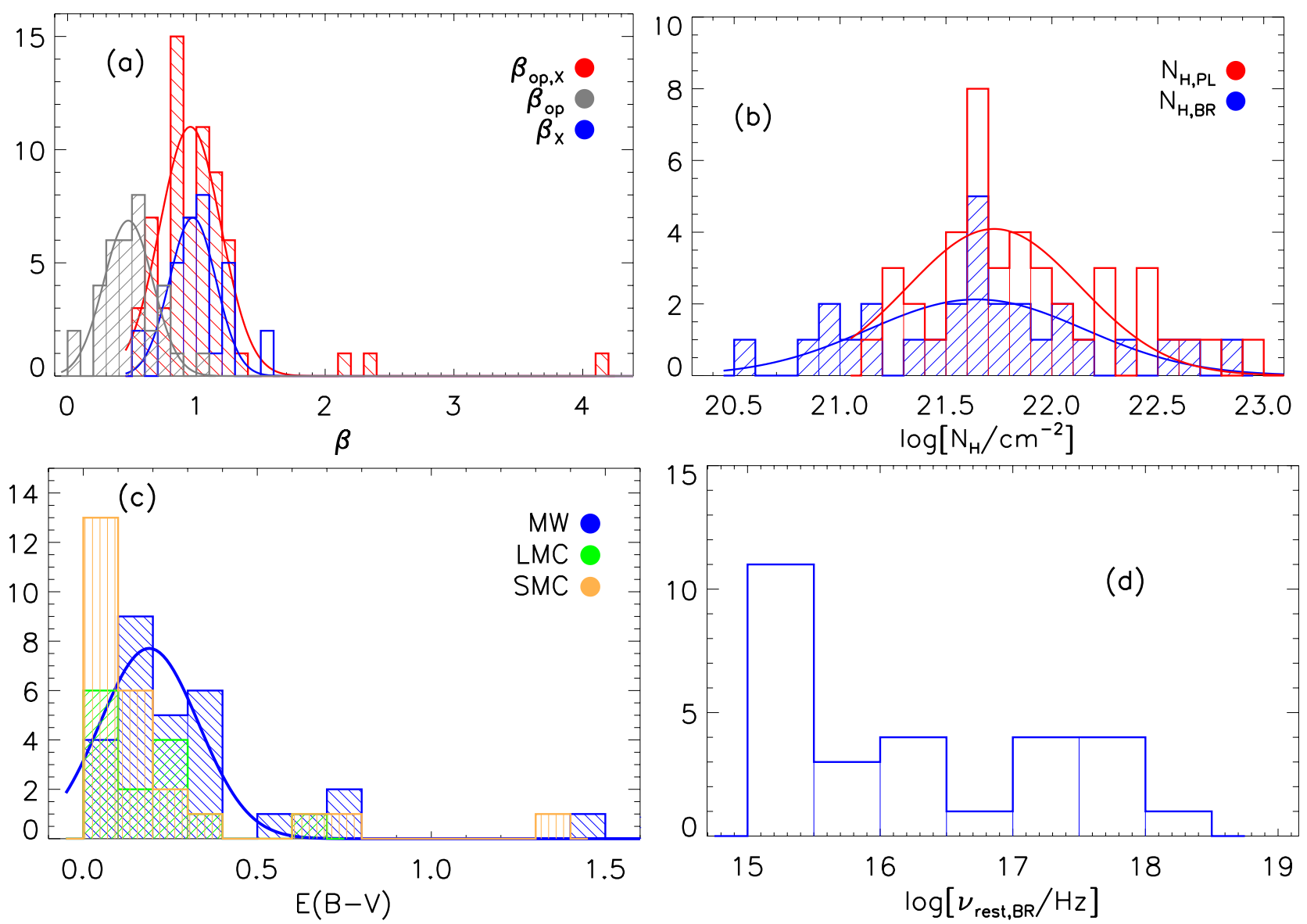

Fig. 2. Parameter distributions. The Color-coding separates different SED best-fitting functions. Top panels. Blue: results obtained by fitting the SEDs with a broken power-law and the relative Gaussian fit (solid line). Red: results obtained by fitting the SEDs with a power-law and the relative Gaussian fit (solid line). a) The spectral indices $(\beta)$ calculated fitting the SED with a single power-law $\left(\beta_{\mathrm{op}, \mathrm{X}}\right)$ and with a broken power-law $\left(\beta_{\mathrm{op}}\right.$, gray, and $\left.\beta_{\mathrm{X}}\right)$. b) The hydrogen column density $\left(N_{\mathrm{H}}\right)$. Bottom panels. c) The optical extinction $(E(B-V))$ distributions separated according to the different extinction laws: MW (blue), LMC (green), and SMC (orange). d) The rest frame break frequency $\left(v_{\text {rest.BR }}\right)$ calculated by fitting the SEDs with a broken-power law.

power-law; in fact the fit is largely weighted over the numerous $\mathrm{X}$-ray data. The mean spectral slope of the optical part of the SED is $\mu\left(\beta_{\text {op }}\right)=0.47 \pm 0.02$, computed by fixing $\beta_{\mathrm{X}}-\beta_{\mathrm{op}}=0.5$, hence it is simply a rigid shift of the distribution.

The distributions of $\beta$ computed over the three different parts of the X-ray LCs (steep decay phase, plateau, normal decay phase) have the following mean values (Figs. 3a,b,c): a) $m\left(\beta_{\mathrm{op}, \mathrm{X}}^{\mathrm{S}}\right)=0.85\left(\right.$ with $\left.\mathrm{SD}=0.22^{12}\right), \mu\left(\beta_{\mathrm{op}, \mathrm{X}}^{\mathrm{P}}\right)=0.92 \pm 0.03$, $\mu\left(\beta_{\mathrm{op}, \mathrm{X}}^{\mathrm{N}}\right)=0.99 \pm 0.03$; b) $\mu\left(\beta_{\mathrm{X}}^{\mathrm{P}}\right)=0.96 \pm 0.02, \mu\left(\beta_{\mathrm{X}}^{\mathrm{N}}\right)=$ $1.00 \pm 0.08$; c) $\mu\left(\beta_{\mathrm{op}}^{\mathrm{P}}\right)=0.49 \pm 0.04, \mu\left(\beta_{\mathrm{op}}^{\mathrm{N}}\right)=0.47 \pm 0.08$. From these distributions we note that the mean spectral index during the plateau is lower than during the normal decay phase, even though they are consistent within $2 \sigma$; in addition, the normal decay spectral index distribution is broader than during the plateau. Therefore we tested the evolution of $\beta$ for each GRB (Fig. 4), with $\beta=\beta_{\mathrm{op}, \mathrm{X}}$ or $\beta=\beta_{\mathrm{X}}$ depending of the fitting function used for each single SED (Table C.8). In most cases the spectrum becomes softer (22 GRBs, red lines), and only for ten GRBs it becomes harder (blue lines). For 26 GRBs we have only one valid SED fit (black dots). If we examine these relationships in the rest frame (inset), in particular only the plateau and normal decay data (magenta dots and orange squares) because we have

\footnotetext{
12 There are too few data to fit a Gaussian over the distribution.
}

only few data for the steep-decay phase (light blue stars) and the unclassified phase (green triangles), then the relationships do not change.

\subsubsection{Hydrogen column density and optical extinction}

The intrinsic hydrogen equivalent column density determines the $\mathrm{X}$-ray absorption and measures the quantity of gas contained in the GRB host galaxy. The origin of this absorption is still debated, but is most likely due to absorption by the intergalactic medium, intervening absorbers or $\mathrm{He}$ in the HII region hosting the GRB (e.g. M 13, Campana et al. 2010, 2012; Behar et al. 2011; Watson et al. 2013).

We calculated the intrinsic hydrogen equivalent column density after subtracting of the MW contribution, both by fitting the $\mathrm{X}$-ray spectrum alone $\left(N_{\mathrm{H}, \mathrm{X}}\right)$ and by a joined fit of optical and X-ray $\operatorname{SED}\left(N_{\mathrm{H}, \mathrm{op}, \mathrm{X}}\right) . N_{\mathrm{H}, \mathrm{op}, \mathrm{X}}$ was computed following the model presented by Morrison \& McCammon (1983), which takes into account the photoelectric cross section per HI-atom units and for solar metallicity (Sect. 2.3). The $N_{\mathrm{H}}$ values found with the two methods are consistent, as shown in Fig. 5, even the low values of $N_{\mathrm{H}}\left(<10^{21} \mathrm{~cm}^{-2}\right)$ are consistent within two sigma.

We therefore restricted our analysis to the intrinsic hydrogen equivalent column densities derived by the optical/X-ray 

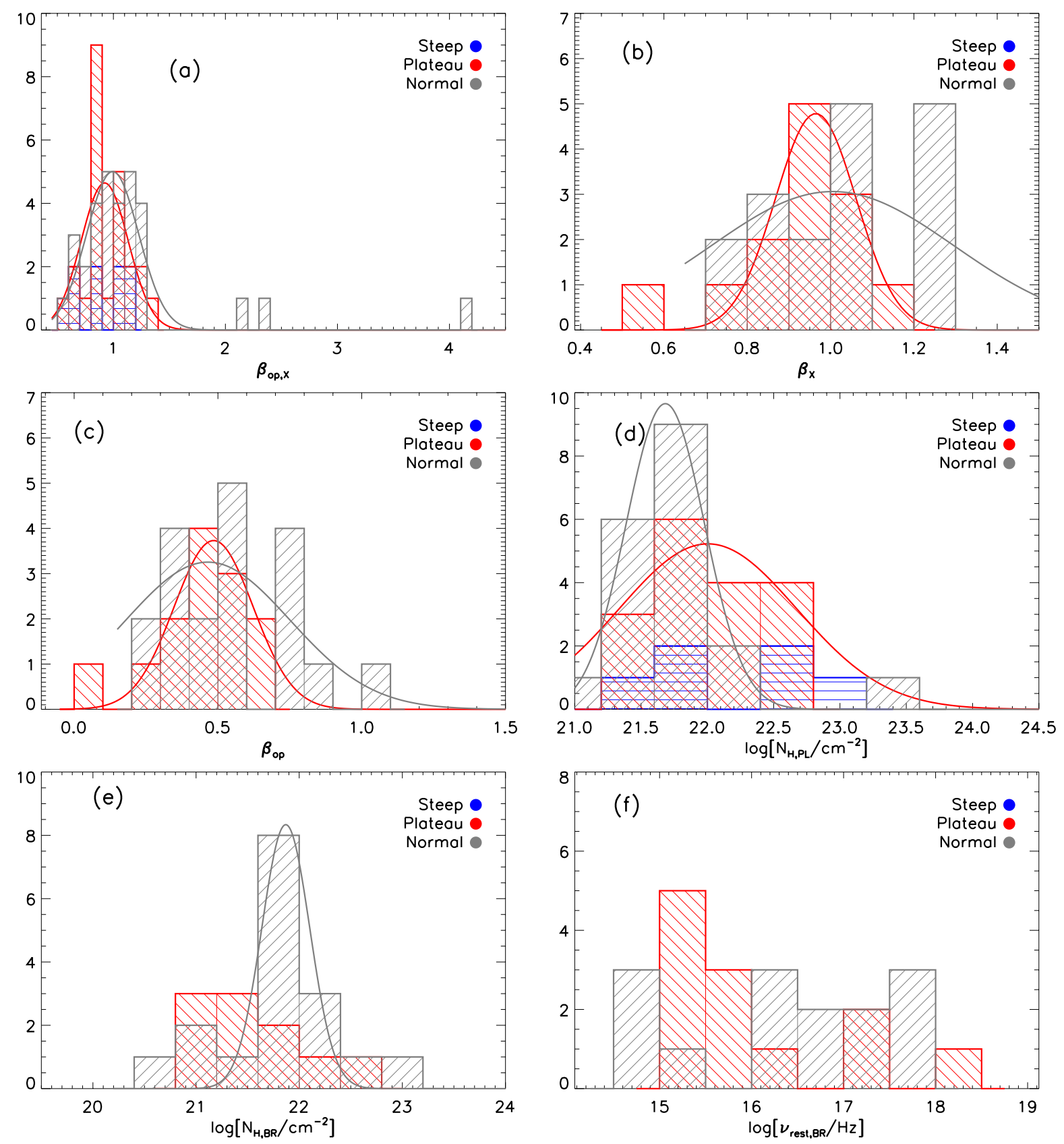

Fig. 3. Parameter distributions considering the X-ray LC part of the SED. Blue lines: steep-decay phase. Red lines: plateau. Gray lines: normal decay phase. a) $\beta_{\mathrm{op}, \mathrm{X}}$ : the spectral slopes calculated using a power-law as fitting function. b) $\beta_{\mathrm{X}}$ and $\left.\mathbf{c}\right) \beta_{\mathrm{op}}$ : the broken power law spectral slopes for the X-ray and optical data, respectively. d) $N_{\mathrm{H}, \mathrm{PL}}$ and e) $N_{\mathrm{H}, \mathrm{BR}}$ : the hydrogen column densities obtained using as SED fitting function a single power-law and a broken power-law, respectively. f) $v_{\text {rest,BR }}$ : the rest frame break frequency calculated fitting the SEDs with a broken-power law.

SEDs $\left(N_{\mathrm{H}, \mathrm{op}, \mathrm{X}} \equiv N_{\mathrm{H}}\right)$. The distributions of the $N_{\mathrm{H}, \mathrm{op}, \mathrm{X}}$ of the host galaxies derived from the single and broken power-law fits are consistent: $\mu\left(\log \left(N_{\mathrm{H}, \mathrm{PL}} / \mathrm{cm}^{-2}\right)\right)=21.60 \pm 0.11$ and $\mu\left(\log \left(N_{\mathrm{H}, \mathrm{BR}} / \mathrm{cm}^{-2}\right)\right)=21.70 \pm 0.06$ (Fig. $\left.2 \mathrm{~b}\right)$. As shown by the distributions of $N_{\mathrm{H}}$ calculated in the different parts of the LCs (Figs. 3d,e), this parameter does not evolve with time because it has a similar mean value, within error, for the steep decay phase, the plateau and, normal decay phase. The values we found for the $N_{\mathrm{H}}$ are consistent with those of M 13 within $1 \sigma$.

In Fig. 2c we show the reddening distributions $(E(B-V))$, differentiating between the best-fitting extinction laws (MW, $\mathrm{LMC}, \mathrm{SMC})$. The mean values are $\mu\left(E(B-V)_{\mathrm{MW}}\right)=0.19 \pm$ $0.02 \mathrm{mag}, m\left(E(B-V)_{\mathrm{LMC}}\right)=0.20 \mathrm{mag}(S D=0.16)$,
$m\left(E(B-V)_{\mathrm{SMC}}\right)=0.20 \mathrm{mag}$, which corresponds to the host galaxy visual extinction $\left(A_{\mathrm{V}}\right) \mu\left(A_{\mathrm{V}, \mathrm{MW}}\right)=0.56 \pm 0.10 \mathrm{mag}$, $m\left(A_{\mathrm{V}, \mathrm{LMC}}\right)=0.63 \mathrm{mag}, m\left(A_{\mathrm{V}, \mathrm{SMC}}\right)=0.59 \mathrm{mag}^{13}$. The mean $A_{\mathrm{V}, \mathrm{SMC}}$ is agrees with the value presented by Zafar et al. (2011).

We studied the properties of the GRB host galaxy environment through the gas-to-dust ratio $\left(N_{\mathrm{H}} / A_{\mathrm{V}}\right.$, Fig. 6). We considered $N_{\mathrm{H}, \mathrm{op}, \mathrm{X}}$ and obtained for different extinction laws $\left.\mu\left(\log \left(N_{\mathrm{H}} / \mathrm{cm}^{-2}\right) /\left(A_{\mathrm{V}} / \mathrm{mag}\right)\right)_{\mathrm{MW}}\right)=21.90 \pm 0.05$ (blue), $\mu\left(\log \left(\left(N_{\mathrm{H}} / \mathrm{cm}^{-2}\right) /\left(A_{\mathrm{V}} / \mathrm{mag}\right)\right)_{\mathrm{LMC}}\right)=22.60 \pm 0.08$ (green) and $\mu\left(\log \left(\left(N_{\mathrm{H}} / \mathrm{cm}^{-2}\right) /\left(A_{\mathrm{V}} / \mathrm{mag}\right)\right)_{\mathrm{SMC}}\right)=21.80 \pm 0.16$ (orange). We compared these results with the $N_{\mathrm{H}} / A_{\mathrm{V}}$ values available

${ }^{13} A_{\mathrm{V}}=R_{\mathrm{V}} E(B-V)$ with $R_{\mathrm{V}}^{\mathrm{MW}}=3.08, R_{\mathrm{V}}^{\mathrm{LMC}}=3.16$ and $R_{\mathrm{V}}^{\mathrm{SMC}}=2.93$ (Pei 1992). 


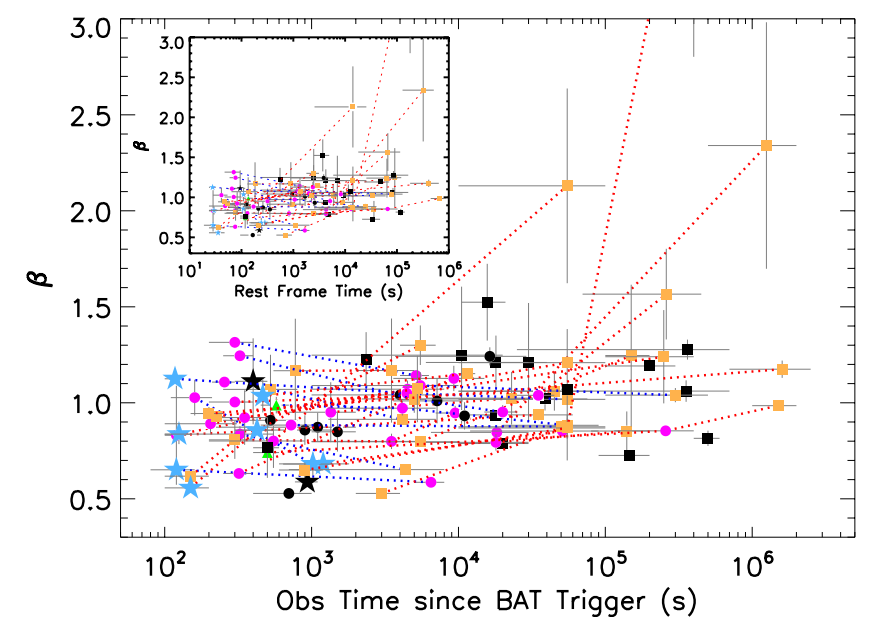

Fig. 4. Evolution of $\beta$ with time for individual GRBs. For every GRB we considered the "correct" spectral index as selected in Table C.8, hence $\beta$ can be $\beta_{\mathrm{op}, \mathrm{X}}$ or $\beta_{\mathrm{X}}$ depending on the chosen SED fitting function, a single power-law or a broken power-law. Blue dotted lines: the initial spectral slope is steeper than the final spectral slope. Red dotted lines: the initial spectral slope is flatter than the final spectral slope. Light blue stars: steep decay data. Magenta dots: plateau data. Orange squares: normal decay data. Black: only one SED is available for these GRBs and precisely during the steep decay (stars), the plateau (dots), and normal decay (squares). Inset: the same as the principal plot, but in the rest frame.

in the literature for the MW, LMC and SMC: $\log \left(\left(N_{\mathrm{H}} / \mathrm{cm}^{-2}\right) /\right.$ $\left.\left(A_{\mathrm{V}} / \mathrm{mag}\right)\right)_{\mathrm{MW}}=21.27$ (Fig. 6, blue star; Bohlin et al. $1978), \log \left(\left(N_{\mathrm{H}} / \mathrm{cm}^{-2}\right) /\left(A_{\mathrm{V}} / \mathrm{mag}\right)\right)_{\mathrm{LMC}}=21.84$ (Weingartner $\&$ Draine 2001) and $\log \left(\left(N_{\mathrm{H}} / \mathrm{cm}^{-2}\right) /\left(\mathrm{A}_{\mathrm{V}} / \mathrm{mag}\right)\right)_{\mathrm{SMC}}=22.19$ (Martin et al. 1989). To compare the Magellanic Clouds data of the $N_{\mathrm{H}}$ from the literature with our results, calculated assuming solar abundances, we converted the values from the literature assuming a metallicity $Z=0.26 Z_{\odot}$ for the LMC and $Z=0.14 Z_{\odot}$ for the SMC (Draine 2003 and references therein). We obtained $\log \left(\left(N_{\mathrm{H}} / \mathrm{cm}^{-2}\right) /\left(A_{\mathrm{V}} / \mathrm{mag}\right)\right)_{\mathrm{LMC}}=21.55$ and $\log \left(\left(N_{\mathrm{H}} / \mathrm{cm}^{-2}\right) /\left(A_{\mathrm{V}} / \mathrm{mag}\right)\right)_{\mathrm{SMC}}=20.99$ (Fig. 6, green and orange stars, respectively).

Our analysis shows that the gas-to-dust ratios of GRBs are higher than the values calculated for the MW, the LMC, and SMC assuming sub solar abundances (e.g. Schady et al. 2010, 2012). We caution, however, that our distributions characterize GRBs that are not heavily absorbed in the X-rays and in the optical band, because our sample is redshift-selected.

\subsubsection{Break frequency}

The rest frame break frequency distribution has a peak around $\log \left(v_{\text {rest,BR }} / \mathrm{Hz}\right) \sim 16$ (Fig. $\left.2 \mathrm{~d}\right)$. The values are spread between the optical and the X-ray band frequencies and it is not possible to fit a Gaussian function to these data.

Because most of our data where taken at late times, they probably correspond to a slow cooling regime for a homogeneous medium, when the break-frequency evolves as $t^{-1 / 2}$ (Sari et al. 1998), moving from the X-ray toward the optical frequencies. Since we cannot follow these changes for a single burst, we tested whether we could find any correlation between the mean time at which we measure the break frequency and the break frequency itself. If GRBs had a similar behavior, the time and the break frequency would be correlated. Figure 7 shows that the peak at low frequencies is spread over a long time interval, and

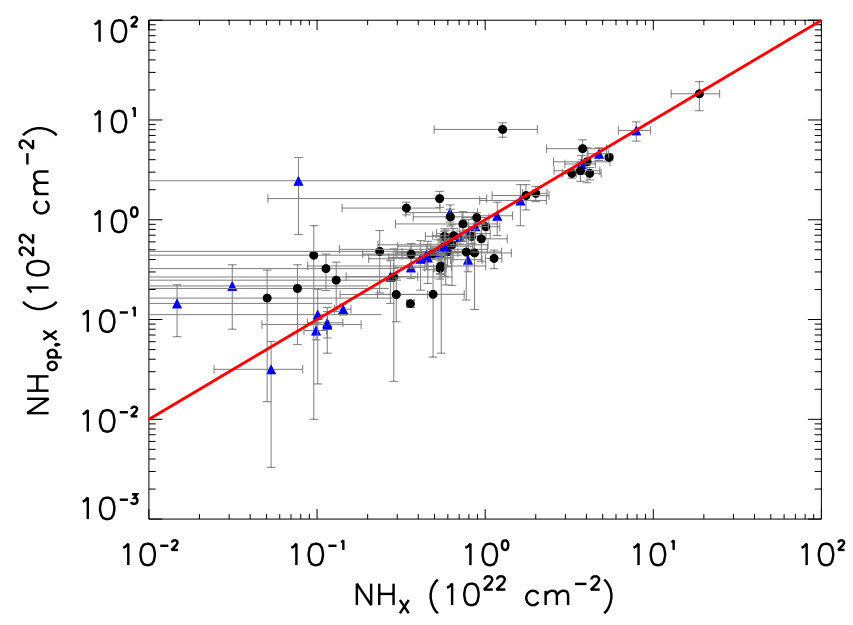

Fig. 5. Comparison between the $N_{\mathrm{H}}$ calculated from the X-ray spectrum $\left(N_{\mathrm{H}, \mathrm{X}}\right)$ and the optical/X-ray $\operatorname{SED}\left(N_{\mathrm{H}, \mathrm{op}, \mathrm{X}}\right)$. Blue triangles stand for the broken power-law fit function and black dots for the simple power-law. Red line: $N_{\mathrm{H}, \mathrm{X}}=N_{\mathrm{H}, \mathrm{op}, \mathrm{X}}$.

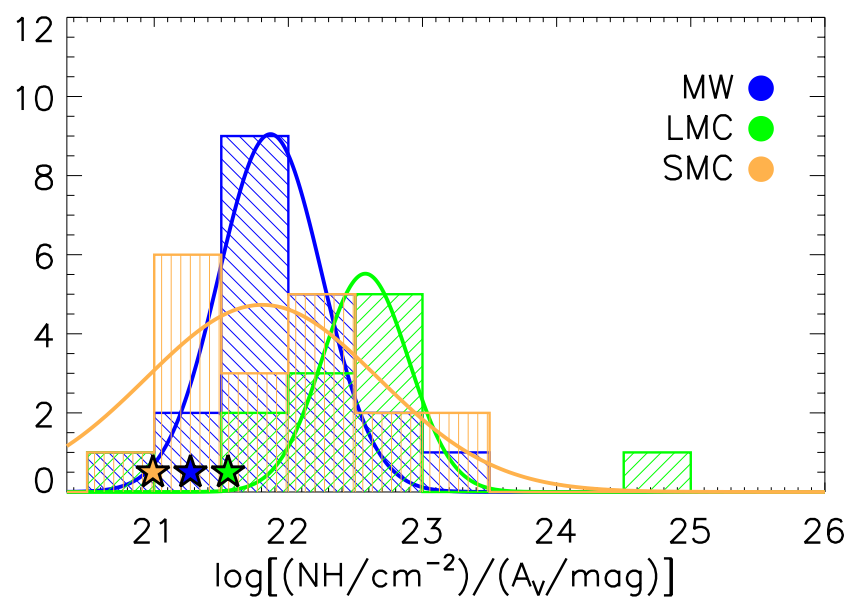

Fig. 6. Distribution of $\log \left(\left(N_{\mathrm{H}} / \mathrm{cm}^{-2}\right) /\left(A_{\mathrm{V}} / \mathrm{mag}\right)\right)$ considering the three different extinction laws used: MW (blue), LMC (green), and SMC (orange). Stars: reference values of the ratios $N_{\mathrm{H}} / A_{\mathrm{V}}$ from the literature. Blue star: $\log \left(\left(N_{\mathrm{H}} / \mathrm{cm}^{-2}\right) /\left(A_{\mathrm{V}} / \mathrm{mag}\right)\right)_{\mathrm{MW}}=21.27$ (Bohlin et al. 1978). Green star: $\left.\log \left(\left(N_{\mathrm{H}} / \mathrm{cm}^{-2}\right) /\left(A_{\mathrm{V}}\right) / \mathrm{mag}\right)\right)_{\mathrm{LMC}}=21.55$ (assuming sub solar abundances). Orange star: $\log \left(\left(N_{\mathrm{H}} / \mathrm{cm}^{-2}\right) /\left(A_{\mathrm{V}} / \mathrm{mag}\right)\right)_{\mathrm{SMC}}=20.99$ (assuming sub solar abundances).

there is no evidence of a correlation between time and frequency (Fig. 7, left). This may be due above all to the dependence of the the break frequency on other parameters, in part to the uncertainties in its measurement, and also because we considered data from different GRBs.

\subsection{Luminosity and energetics}

In Fig. 8 we plot the $\mathrm{X}$-ray ( $1 \mathrm{keV}$, gray lines) and optical ( $R$ band) blue lines) rest-frame LCs of the GRBs in our sam$\mathrm{ple}^{14}$. The optical and X-ray LCs have similar luminosities; our redshift-selected sample favors bright optical GRBs.

\footnotetext{
${ }^{14}$ The X-ray and optical data are k-corrected. Optical data are not corrected for Galactic and host galaxy absorption; X-ray data are corrected for Galactic and host galaxy absorption. Therefore, the optical luminosity derived is a lower limit of the real value. However, since the GRBs considered have smaller absorption (see Sect. 3.1.2) the real luminosity is about a factor 2 higher than the one considered.
} 

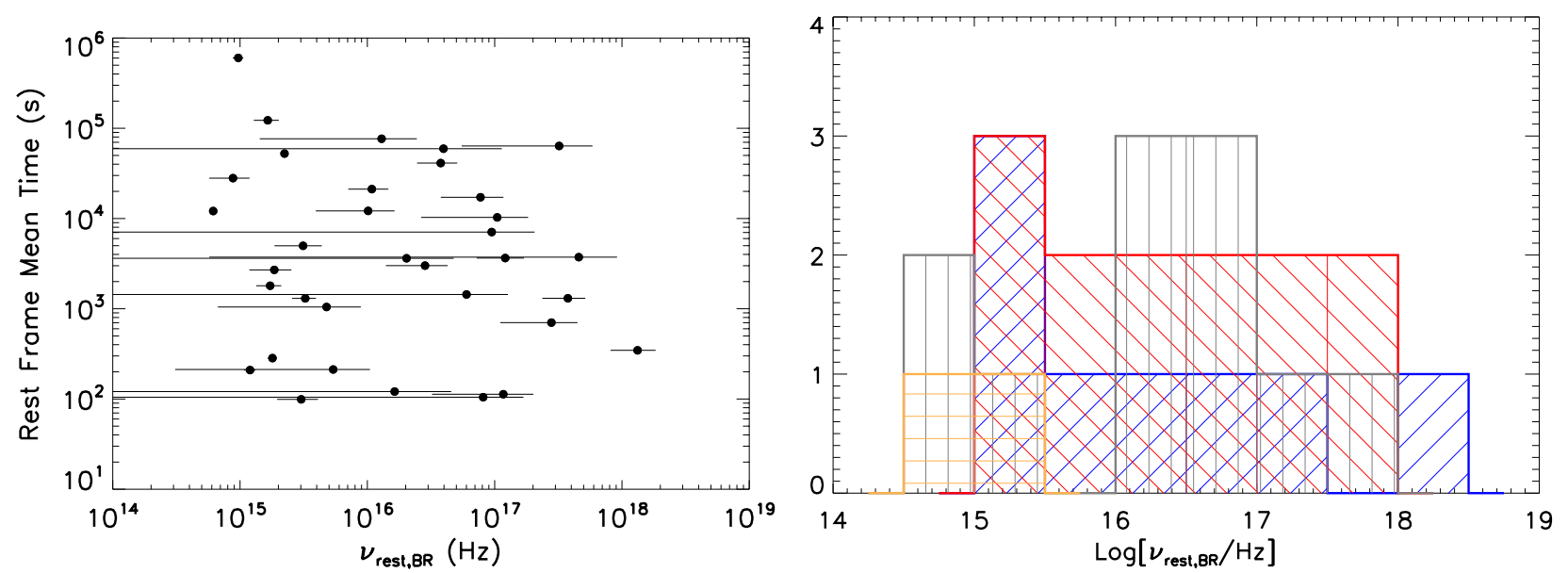

Fig. 7. Break frequency. Left: break frequency $\left(v_{\text {rest,BR }}\right)$ vs. the mean time $\left(t_{\text {rest, }, \mathrm{m}}\right)$ of the interval in which the SED is calculated. Right: the distribution of the break frequencies. Blue: $t_{\text {rest, } \mathrm{m}}<500 \mathrm{~s}$. Red: $500<t_{\text {rest, } \mathrm{m}}<10^{4} \mathrm{~s}$. Gray: $10^{4}<t_{\text {rest, } \mathrm{m}}<10^{5} \mathrm{~s}$. Orange: $t_{\text {rest,m }}>10^{5} \mathrm{~s}$. The time intervals have been arbitrarily chosen.

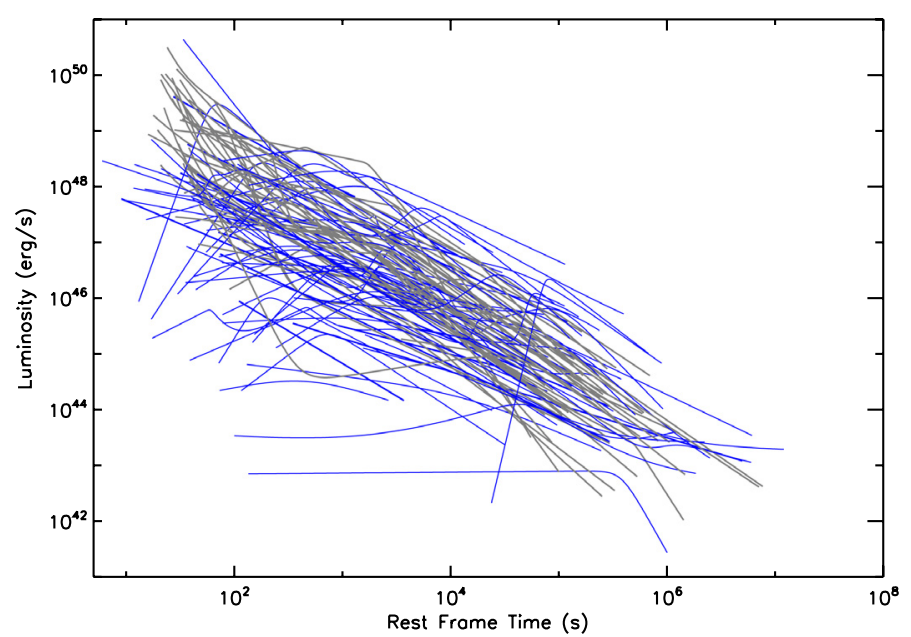

Fig. 8. X-ray (1 keV, gray) and optical ( $R$ band, blue) LCs in the rest frame.

For the GRBs in our sample, we compared the optical ( $R$ band) and X-ray (at $1 \mathrm{keV}$ ) flux (Fig. 9) in a common rest frame time interval (920-1200 s): the X-ray emission $\left(\log \left(\mu\left(F_{\mathrm{X}}\right) /\left(\operatorname{erg~cm} \mathrm{cm}^{-2} \mathrm{~s}^{-1}\right)\right)=-12.54, \sigma=0.49\right)$ is on average one order of magnitude fainter than the optical $\left(\log \left(\mu\left(F_{\mathrm{op}}\right) /\left(\mathrm{erg} \mathrm{cm}^{-2} \mathrm{~s}^{-1}\right)\right)=-11.41, \sigma=0.34\right)$.

The optical LCs can show an early-time rise or a quasiconstant phase (optical plateau), followed by a decay. Panaitescu \& Vestrand (2011) claimed that there are some correlations involving the energies and luminosities calculated at the peak of the early-time rise or at the end of the optical plateau, which are predicted by theoretical models. We verified these relations in the observer and the rest frames considering the GRBs with an optical LC with an initial peak and with an initial optical plateau (Table 3).

To compute the relations between two parameters, we used the best-fitting procedure, which accounts for the sample variance (D'Agostini 2005). All results are listed in Table 2 and presented in Figs. 10-12.

We confirm the correlation between the optical energy $(L \times$ $t_{\text {rest }}$ with $L=L_{\mathrm{end}}, L_{\mathrm{pk}}$ ) and the isotropic gamma-ray energy ${ }^{15}$

\footnotetext{
$\overline{15}$ We used the values presented in M 13.
}

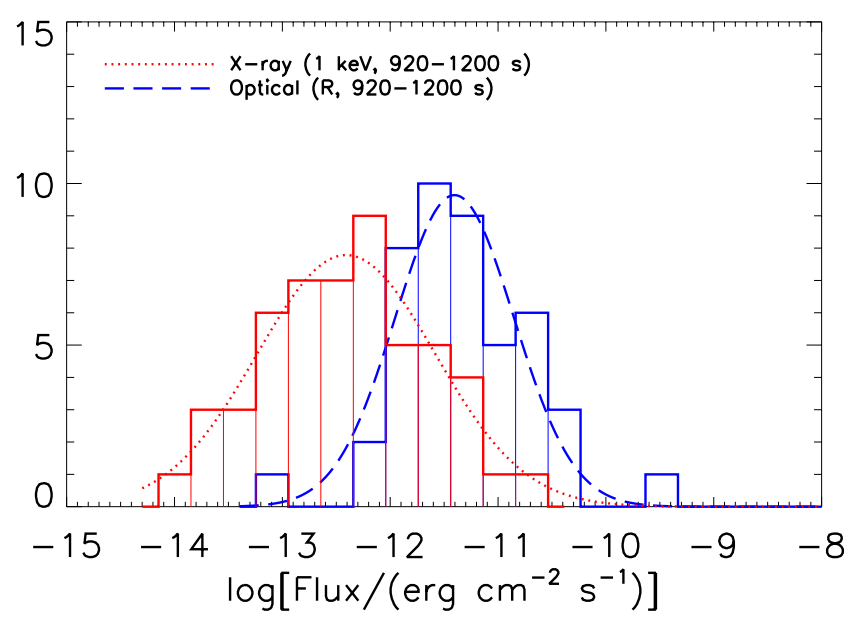

Fig. 9. Energetics. Distribution of the X-ray ( $1 \mathrm{keV}$, red, solid line) and optical ( $R$ band, blue, solid line) flux calculated in a common rest frame time interval (920-1200 s) for our sample and their distributions (red, dotted line and blue, dashed line, respectively).

( $E_{\gamma, \text { iso }}$, Amati et al. 2008) and the optical energy and the energy calculated in the BAT energy band 15 ( $E_{\gamma}^{15-150}$; Fig. 10$)$. However, for all these correlations the data show very broad distributions.

There is a weak indication that the optical plateau end luminosities and the relative observer and rest frame times are correlated (Fig. 11, left). The same occurs for the peak luminosities and the relative observer and rest frame times (Fig. 11, right). Since there are few elements in our sample, the correlation is not reliable.

In the observer frame, the peak flux correlates with the peak time (Fig. 12, bottom), but the optical plateau end fluxes and their times are not related (Fig. 12, top). We had only few data for this as well: 19 GRBs for the peak relation and 14 for the plateau, and a few discrepant cases.

We also measured the optical luminosity distributions at four different rest frame times: $500 \mathrm{~s}, 1 \mathrm{~h}, 11 \mathrm{~h}$, and 1 day. The 11-h time frame is commonly chosen because it is reasonable to assume that the cooling frequency has not passed the optical band yet (Freedman \& Waxman 2001; Piran et al. 2001), in addition, $11 \mathrm{~h}$ and 1 day are approximately the 
Table 2. Two-parameter correlations involving optical luminosities and fluxes.

\begin{tabular}{lccccccc}
\hline \hline$X$ & $Y$ & $m$ & $q$ & $\sigma$ & $\rho$ & $K$ & $R$ \\
\hline$L \times t_{\text {rest }}$ & $E_{\gamma}^{15-150}$ & $0.65 \pm 0.03$ & $-0.39 \pm 2.52$ & $0.59 \pm 0.01$ & 0.64 & 0.47 & 0.72 \\
$L \times t_{\text {rest }}$ & $E_{\gamma, \text { iso }}$ & $0.78 \pm 0.05$ & $-4.42 \pm 4.99$ & $0.58 \pm 0.03$ & 0.72 & -0.49 & 0.80 \\
$t_{\text {rest,PL }}$ & $L_{\text {end,PL }}$ & $-0.83 \pm 0.14$ & $8.64 \pm 1.36$ & $0.84 \pm 0.06$ & -0.66 & -0.51 & -0.65 \\
$t_{\text {obs,PL }}$ & $L_{\text {end,PL }}$ & $-0.77 \pm 0.18$ & $8.81 \pm 2.35$ & $0.90 \pm 0.07$ & -0.55 & -0.41 & -0.55 \\
$t_{\text {rest,PK }}$ & $L_{\text {end,PK }}$ & $-1.40 \pm 0.13$ & $10.47 \pm 0.81$ & $0.92 \pm 0.04$ & -0.79 & -0.64 & -0.77 \\
$t_{\text {obs,PK }}$ & $L_{\text {end,PK }}$ & $-1.32 \pm 0.20$ & $10.86 \pm 0.75$ & $1.08 \pm 0.06$ & -0.66 & -0.48 & -0.67 \\
$t_{\text {obs,PL }}$ & $F_{\mathrm{PL}}$ & $-0.48 \pm 0.10$ & $-12.09 \pm 1.19$ & $0.65 \pm 0.03$ & -0.39 & -0.26 & -0.53 \\
$t_{\text {obs,PK }}$ & $F_{\mathrm{PK}}$ & $-0.93 \pm 0.08$ & $-10.14 \pm 0.72$ & $0.69 \pm 0.02$ & -0.78 & -0.62 & -0.71 \\
\hline
\end{tabular}

Notes. From left to right: $X$ and $Y$ parameters to be correlated (the best-fitting law reads $\log (Y)=q+m \log (X)$ ); best-fitting parameters as obtained accounting for the sample variance (D'Agostini 2005): slope $(m)$, normalization $(q)$, intrinsic scatter $(\sigma)$; errors are given at $95 \%$ c.l. The last three columns list the value of the Spearman rank $(\rho)$, Kendall coefficient $K$, and $R$-index $r$ statistics.
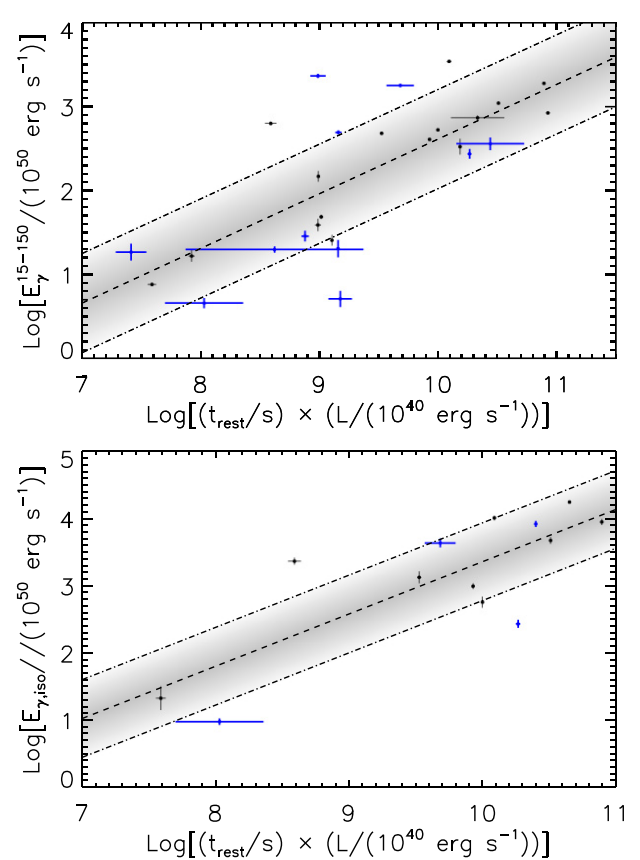

Fig. 10. Relations between the optical energy (time $\times$ luminosity) of the optical plateau end (blue dots) and of the peak (black dots) and the 15-150 keV BAT energy (top) and the isotropic prompt emission energy (bottom). Dashed line: best-fitting power-law model obtained accounting for the sample variance (D'Agostini 2005). Gray area: the $68 \%$ confidence region around the best fit. The results of the fit are listed in Table 2.

times at which several authors found a bimodal distribution ${ }^{16}$ of the luminosities (Liang \& Zhang 2006; Nardini et al. 2006; Kann et al. 2006; Nardini et al. 2008). We found no bimodal distribution in our data, as asserted in recent studies (Melandri et al. 2008b; Oates et al. 2009, 2011; Kann et al. 2010b, 2011). The mean luminosity simply decreases with time (Fig. 13): $\mu\left(\log \left(L_{500 \mathrm{~s}} / \mathrm{erg} \mathrm{s}^{-1}\right)\right)=45.90 \pm$ 0.06 (64 GRBs), $\mu\left(\log \left(L_{1 \mathrm{~h}} / \mathrm{erg} \mathrm{s}^{-1}\right)\right)=45.40 \pm 0.06$ (57 GRBs), $\mu\left(\log \left(L_{11} \mathrm{~h} / \mathrm{erg} \mathrm{s}^{-1}\right)\right)=44.50 \pm 0.07$ (40 GRBs) and $\mu\left(\log \left(L_{1 \text { day }} / \mathrm{erg} \mathrm{s}^{-1}\right)\right)=44.20 \pm 0.09(32 \mathrm{GRBs})($ Table 1$)$.

\footnotetext{
${ }^{16}$ Possibly caused by a bimodality in the optical luminosity function or by the absorption of gray dust in a fraction of bursts (Nardini et al. 2008).
}

\subsection{The optical LCs}

The optical LCs show different shapes and features (Table 3; see also Li et al. 2012, their Fig. 2). For each GRB, we selected the optical LC observed with the filter with the widest temporal coverage and the most reliable fit (Table C. $1^{17}$ ).

In general, they have a rising or constant part, which can occur at any time. This occurs for 53 GRBs in our sample. Only ten GRBs show a simple power-law trend and five GRBs have an LC with an initial decay followed by an almost constant optical flux. The optical LCs with a single power-law decay have an initial time $>600 \mathrm{~s}$ in the observer frame (GRB 050824 $4^{18}$, GRB 050908, GRB 060502A) or they are poorly sampled (GRB 050904, GRB 051111, GRB 080721, and GRB 091018). GRB 050401 and GRB 050922C show weak variability in their optical LCs, even though their best-fit function is a simple power-law. GRB 060912A has a well-sampled optical LC, fitted with a single power-law. Of the five GRBs with an initial decay followed by almost constant flux, GRB 060908 and GRB 090424 show a shallow phase at late times $\left(\gtrsim 10^{6}\right)$, which may be due to the host galaxy. For GRB 061126, GRB 070529, and GRB 090102 the LC break occurs at $\sim 10^{3}-10^{4} \mathrm{~s}$ (observer frame). For these five GRBs the initial time of the optical observations is $\sim 100 \mathrm{~s}$. Even though these GRBs do not show variable LCs, we do not know what happened before the observations; they could have a very variable LC similar to GRB 080319B (Racusin et al. 2008).

There are 14 GRBs with an optical LC with an early peak (i.e., an initial rise followed by a decay) and 14 with a quasiconstant phase (i.e., optical plateaus, an initial quasi-constant phase followed by a decay). The optical plateaus and rises in the LCs are interpreted as the onset of the forward shock emission, when the blast wave decelerates: the peaked LCs correspond to an impulsive ejecta release, where all ejecta have the same Lorentz factor after the burst phase; the plateaus are caused by the energy injection in the forward-shock due to an extended ejecta release, a wide distribution of the ejecta initial Lorentz factors or both (e.g. Panaitescu \& Vestrand 2011; Oates et al. 2009), or the onset of the afterglow for the wind medium (e.g. Chevalier \& Li 1999; Ghirlanda et al. 2012).

Sixteen GRBs show a late-time re-brightnening (i.e., at late times the LC displays a rise phase followed by a decay phase

\footnotetext{
17 The complete and machine-readable form of the table is provided at CDS (table1c.dat).

${ }^{18}$ In this case we consider the overall trend, since it is difficult to fit the LC with a more complicated fit function. For a detailed study of this LC see Sollerman et al. (2007).
} 

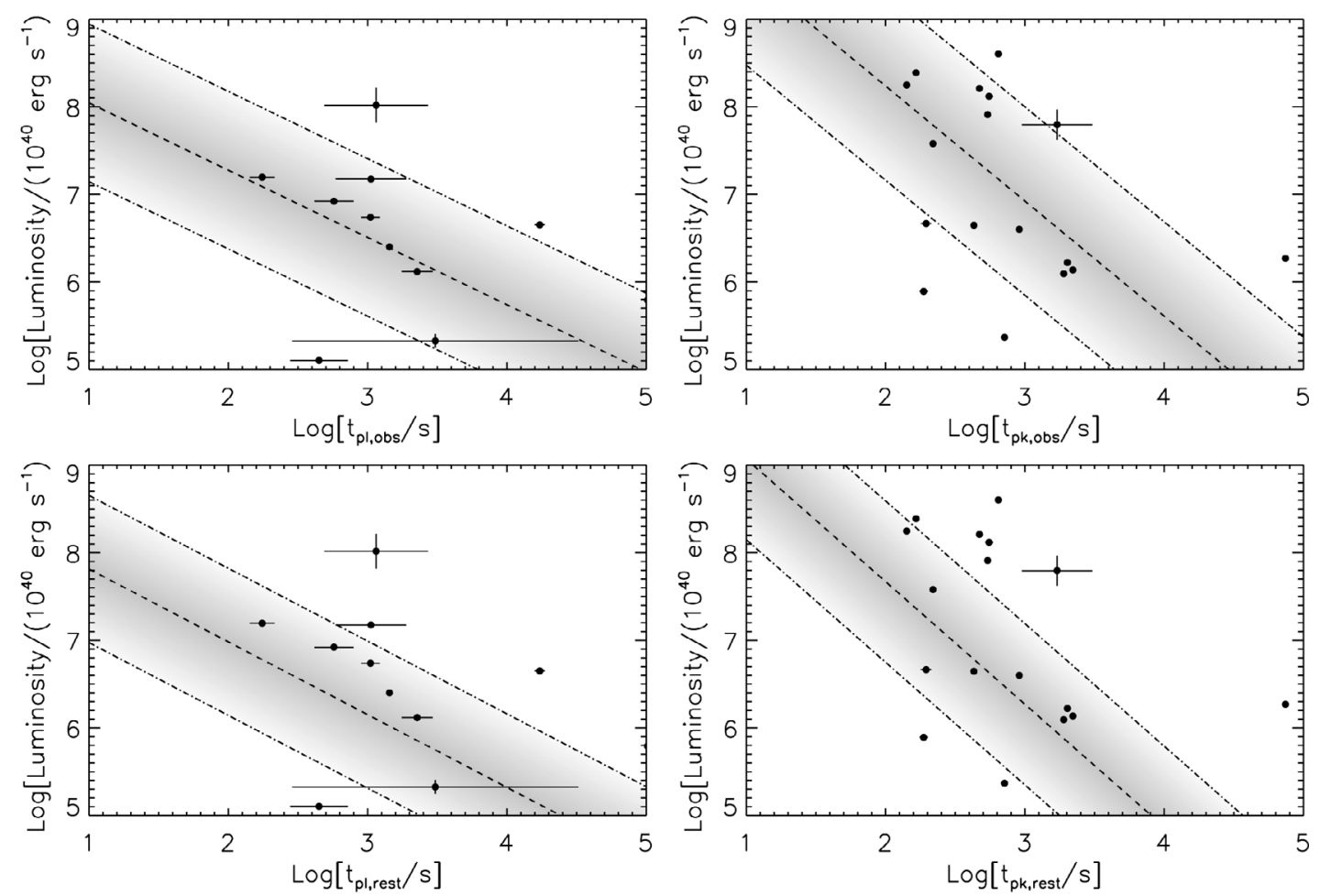

Fig. 11. Relations between the optical luminosity of the end of the plateau (left) and of the peak (right) and the relative observer (top) and rest (bottom) frame time. Dashed line: best-fitting power-law model obtained accounting for the sample variance (D'Agostini 2005). Gray area: the $68 \%$ confidence region around the best fit. The results of the fit are listed in Table 2.

with the same slope as before). The re-brightening may be related to the jet structure, and seems to agree with the on-axis two-component jet model, with the re-brightening corresponding to the emergence of the slow component (e.g. Jakobsson et al. 2004; Racusin et al. 2008; Liang et al. 2012).

Three GRBs show a series of initial large bumps (i.e., more than one peak). GRB 060904B shows two bumps during the $\mathrm{X}$-ray plateau and a shallow decay starting roughly at the beginning of the X-ray normal decay. The two optical peaks are not correlated with the high-energy emission and the subsequent optical bump is assumed to trace the onset of the forward shock (Rykoff et al. 2009). The optical LC of GRB 060906 has two bumps that coincide with the X-ray plateau. These bumps could be associated to a change of the circumburst density (Lazzati et al. 2002; Cenko et al. 2009). The GRB 080928 optical LC was modeled by multiple energy injections into the forward shock, and not with the central engine, since the fluctuations occur on a long timescale (Rossi et al. 2011). The first peak is assumed to be the onset of the afterglow, while the following two bumps are produced by the central engine activity (Rossi et al. 2011).

Five optical LCs show small bumps (i.e., weak fluctuations over the power-law decay). The optical bumps could be related to the erratic late-time central engine activity ( $\mathrm{Li}$ et al. 2012).

The optical LCs have a complex behavior, and during a welldefined X-ray LC phase the optical LC can rise and then decay, or vice versa. Specifically ${ }^{19}$ :

- Steep decay: $42 \%$ of the optical LCs rise, $32 \%$ decay, $16 \%$ are constant, and $10 \%$ have a complex behavior (rise-decay, bumps).

\footnotetext{
19 The percentage refers to single LC parts, not to the total number of GRBs.
}
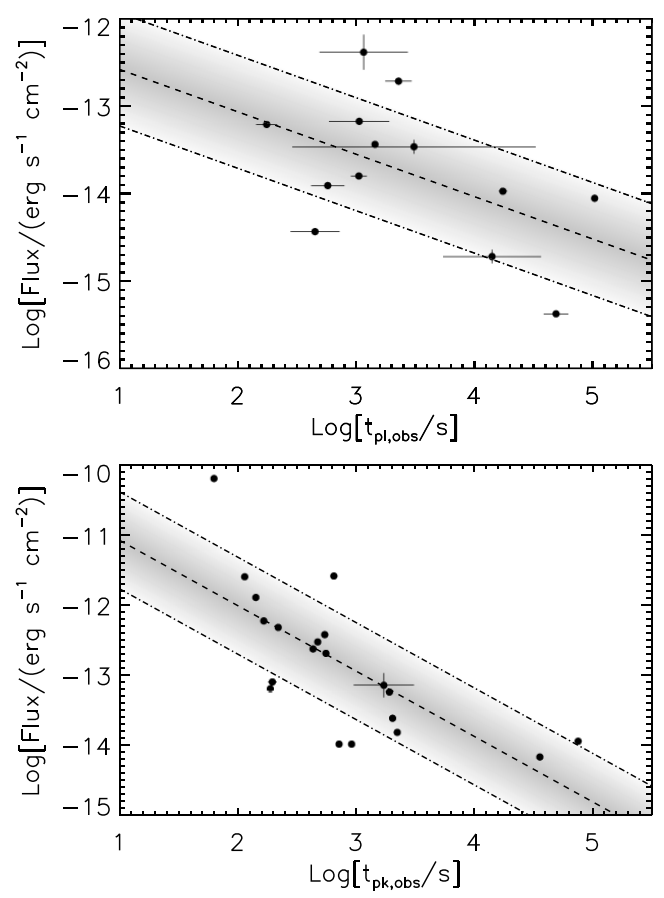

Fig. 12. Relations between the optical flux and the observer time of the end of the plateau (top) and the peak (bottom). Dashed line: bestfitting power-law model obtained accounting for the sample variance (D'Agostini 2005). Gray area: the 68\% confidence region around the best fit. The results of the fit are listed in Table 2.

- Plateau: $16 \%$ of the optical LCs rise, $47 \%$ decay, $8 \%$ are constant, $26 \%$ rise and decay and $4 \%$ have one or more bumps. 
E. Zaninoni et al.: The gamma-ray burst optical light-curve zoo: comparison with the X-ray observations
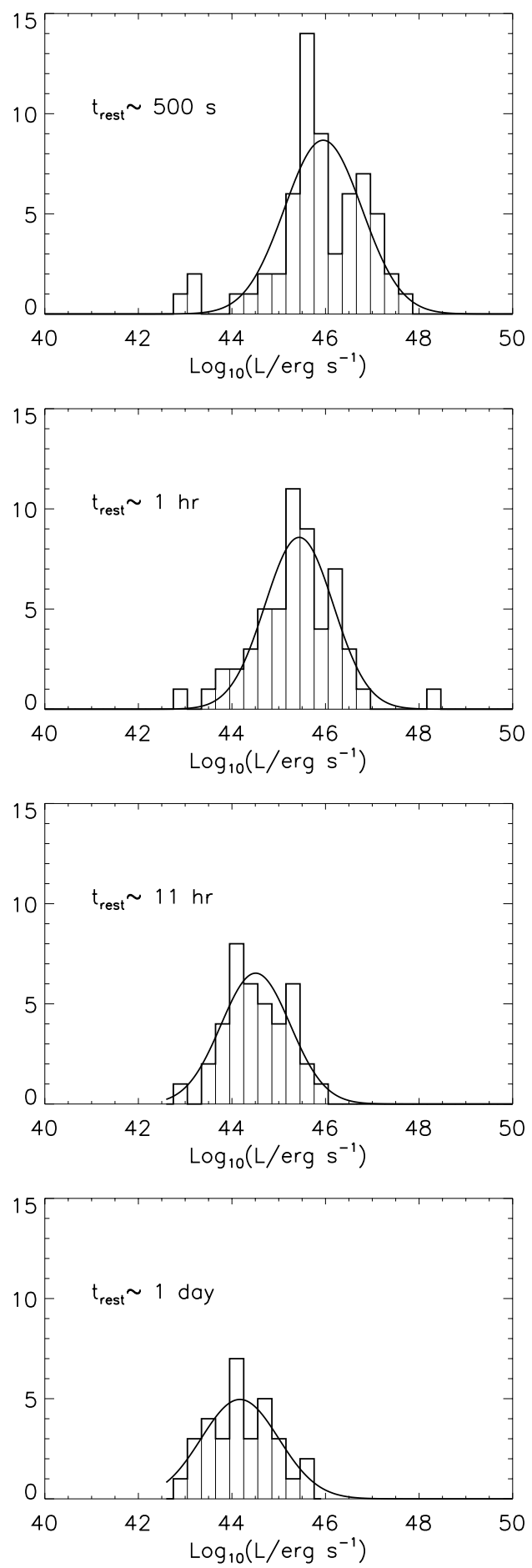

Fig. 13. Distribution of the optical $R$ luminosity calculated for four different rest frame times: $500 \mathrm{~s}, 1 \mathrm{~h}, 11 \mathrm{~h}$, and 1 day. The black solid line corresponds to the Gaussian fit of the data. The results are listed in Table 1.

- Normal decay: $77 \%$ of the optical LCs decay and $23 \%$ rise and decay or have a more complex behavior.

The complexity of the LCs decreases as a function of time.

\subsection{Comparison between the optical and X-ray LCs}

For every GRB we compared the optical LC slopes with the contemporaneous X-ray LC slopes. For both the optical and the
Table 3. Subdivision of the GRBs in our sample according to the optical LC features.

\begin{tabular}{|c|c|c|c|c|}
\hline \multicolumn{5}{|c|}{ Initial peak } \\
\hline $050730^{a}$ & $050820 \mathrm{~A}^{a}$ & 060418 & 060605 & 060607A \\
\hline $060614^{a}$ & 061007 & 061121 & 070318 & 070419A \\
\hline $070802^{a}$ & 071025 & 071031 & $071112 \mathrm{C}$ & 080603A \\
\hline $080710^{a}$ & 080810 & 081008 & 081203A & \\
\hline \multicolumn{5}{|c|}{ Initial shallow phase } \\
\hline 050408 & 050416A & 060124 & 060210 & 060526 \\
\hline 060729 & 070125 & 070208 & 070411 & 071010A \\
\hline 080330 & 090313 & 090426 & 090618 & \\
\hline \multicolumn{5}{|c|}{ Late-time re-brightening } \\
\hline 050820A & 060206 & 060526 & 060927 & 061121 \\
\hline 071003 & 071010A & 080310 & 080607 & 080913 \\
\hline 081008 & 081029 & 091127 & $100418 \mathrm{~A}$ & $100901 \mathrm{~A}$ \\
\hline \multicolumn{5}{|c|}{ Series of initial large bumps } \\
\hline 060904B & 060906 & 080928 & & \\
\hline \multicolumn{5}{|c|}{ Small bumps } \\
\hline $050401^{b}$ & 060607A & 071025 & 071031 & $090313^{b}$ \\
\hline
\end{tabular}

Notes. ${ }^{(a)}$ At late times. ${ }^{(b)}$ For the $R_{\mathrm{C}}$ observations.

X-ray LCs, we considered only the continuum part of the LC, excluding small bumps and flares (see M 13). As in the previous section, for each GRB, we selected the optical LC observed with the filter with the widest temporal coverage and the most reliable fit (Table C. $1^{20}$ ); the X-ray LC parameters are those derived in M 13. From the synchrotron spectrum (Sari et al. 1998), if $v_{\mathrm{c}}<v_{\mathrm{op}}<v_{\mathrm{X}}$, with $v_{\mathrm{c}}$ the cooling frequency, the difference between the contemporaneous optical and X-ray slopes is $\Delta \alpha=0$. If $v_{\mathrm{op}}<v_{\mathrm{c}}<v_{\mathrm{X}}$, for the slow cooling regime, $\Delta \alpha= \pm 1 / 4^{21}$. We subdivided our sample into three groups, depending on whether the pairs of the optical/X-ray slopes follow the relation $\Delta \alpha=0,1 / 4$, or not at all within $1 \sigma^{22}$ (Table 4 and Fig. 14):

- Group A: all pairs of slopes of the same GRB satisfy the relation $\Delta \alpha=0, \pm 1 / 4$ (13 GRBs).

- Group B: some slopes of the same GRB satisfy the relation $\Delta \alpha=0, \pm 1 / 4$ (27 GRBs).

- Group C: no slopes of the same GRB satisfy the relation $\Delta \alpha=0, \pm 1 / 4$ (28 GRBs).

Some X-ray LCs show an initial steep decay; this is generally not present in the optical LCs, which display a rise, a plateau, or a normal decay. The X-ray steep decay is well explained as the decay of the prompt emission, and its slope value is particularly sensitive to the chosen zero time of the power-law decay, $t_{0} \sim 0$ (e.g. the BAT trigger time) or $t_{0}=t_{90}$ (for details see M 13). For this reason, the steep-decay phase was not considered in our classification.

We plot $\alpha_{\mathrm{X}}$ vs. $\alpha_{\mathrm{op}}$ for every GRB in Fig. 15 (e.g. Urata et al. 2007). About half of the $\alpha_{\mathrm{X}}$ vs. $\alpha_{\mathrm{op}}$ couples refer to the X-ray LC normal decay phase and the other half to the plateau. We noted

20 The complete and machine-readable form of the table is provided at CDS (table1c.dat).

${ }^{21}$ This is valid in the slow cooling regime for the constant interstellar medium (ISM) and the wind case: for $v_{\mathrm{m}}<v<v_{\mathrm{c}}, \alpha_{1}=3(\mathrm{p}-1) / 4$, and for $v>v_{\mathrm{c}}, \alpha_{2}=(3 p-2) / 4$ in the ISM case, so $\alpha_{1}-\alpha_{2}=(3 p-3-3 p+$ 2) $/ 4=-1 / 4$; for the wind case, for $v_{\mathrm{m}}<v<v_{\mathrm{c}}, \alpha_{1}=(3 p-1) / 4$, and for $v>v_{\mathrm{c}}, \alpha_{2}=(3 p-2) / 4$, so $\alpha_{1}-\alpha_{2}=(3 p-1-3 p+2) / 4=1 / 4$.

22 A similar method was used by Panaitescu \& Vestrand (2011) to classify coupled and decoupled LCs. 
Table 4. List of GRBs in the three groups.

\begin{tabular}{|c|c|c|c|c|}
\hline \multicolumn{5}{|l|}{$G R B$} \\
\hline \multicolumn{5}{|c|}{ Group A (13 GRBs) } \\
\hline 050416A & 050824 & 050904 & 060912A & 061007 \\
\hline 080310 & 080319B & 080330 & 080603A & 080607 \\
\hline 080913 & 100418A & $100901 \mathrm{~A}$ & & \\
\hline \multicolumn{5}{|c|}{ Group B (27 GRBs) } \\
\hline 050319B & 050401 & 050408 & $050525 \mathrm{~A}$ & 051109A \\
\hline 060124 & 060502A & 060512 & 060526 & 060614 \\
\hline 060904B & 060908 & 061121 & 060729 & 070208 \\
\hline 071003 & 071031 & 071010A & 080413B & 080710 \\
\hline 080928 & 081008 & 081203A & 090313 & 090618 \\
\hline 091018 & 090926A & & & \\
\hline \multicolumn{5}{|c|}{ Group C (28 GRBs) } \\
\hline 050730 & 050820A & 050908 & 050922C & 051111 \\
\hline 060206 & 060210 & 060418 & 060605 & 060607A \\
\hline 060906 & 060927 & 061126 & 070125 & 070318 \\
\hline 070411 & 070419A & 070529 & 070802 & 071025 \\
\hline 071031 & $071112 \mathrm{C}$ & 080721 & 080810 & 081029 \\
\hline 090102 & 090424 & 090426 & 091127 & \\
\hline
\end{tabular}

Notes. See Appendix B for more details.

that the GRBs in Group A and B have more complex LCs than the Group C GRBs, indeed most of the X-ray and optical LCs of Group A and B GRBs have Type IIa or III shapes. Therefore, when GRBs have LCs that are well sampled and have a good time coverage, hence with more complicated shapes, the X-ray and the optical LCs show a similar trend. When we have fewer data, we cannot compare some parts of the LCs and perhaps the observed slope is different from the real behavior of the LC.

$\mathrm{X}$-ray flares do not influence the relation between the X-ray and optical LCs, because in Group A there are 5/14 GRBs with flares (36\%), in Group B there are 8/26 (31\%), and in Group C are $8 / 28(29 \%)$. This agrees with the percentage found in other samples (Chincarini et al. 2010a; Margutti et al. 2013).

\section{Discussion}

We presented the analysis of a large and homogeneous data set, useful for studying the GRB rest frame properties and for comparing the optical and X-ray emission.

The comparison between the X-ray and the optical LCs and SEDs enables us to investigate the nature of their emission mechanism and to verify if they have the same origin. For the internalexternal shock model (Sari et al. 1998), the forward shock propagating into the external medium gives rise to the X-ray and optical emission. If the optical and the X-ray LCs have similar shapes and slopes, they could be caused by synchrotron emission and probable are produced by the forward shock (e.g. Zhang et al. 2006). Indeed, the X-ray emission is mainly influenced by the central engine activity: the steep decay is thought to be the tail of the prompt emission (Kumar \& Panaitescu 2000) or it is direct emission from the central engine (Barniol Duran \& Kumar 2009). The plateau reflects the effect of energy injection into the forward shock (e.g. Zhang et al. 2006).

The optical LCs show various features: initial peaks or constant phases, which are probably caused by the onset of the forward shock (e.g. Panaitescu \& Vestrand 2011), late-time rebrightenings that may depend on the structure of the jet (e.g. Racusin et al. 2008), and small bumps linked to the central engine activity ( $\mathrm{Li}$ et al. 2012).
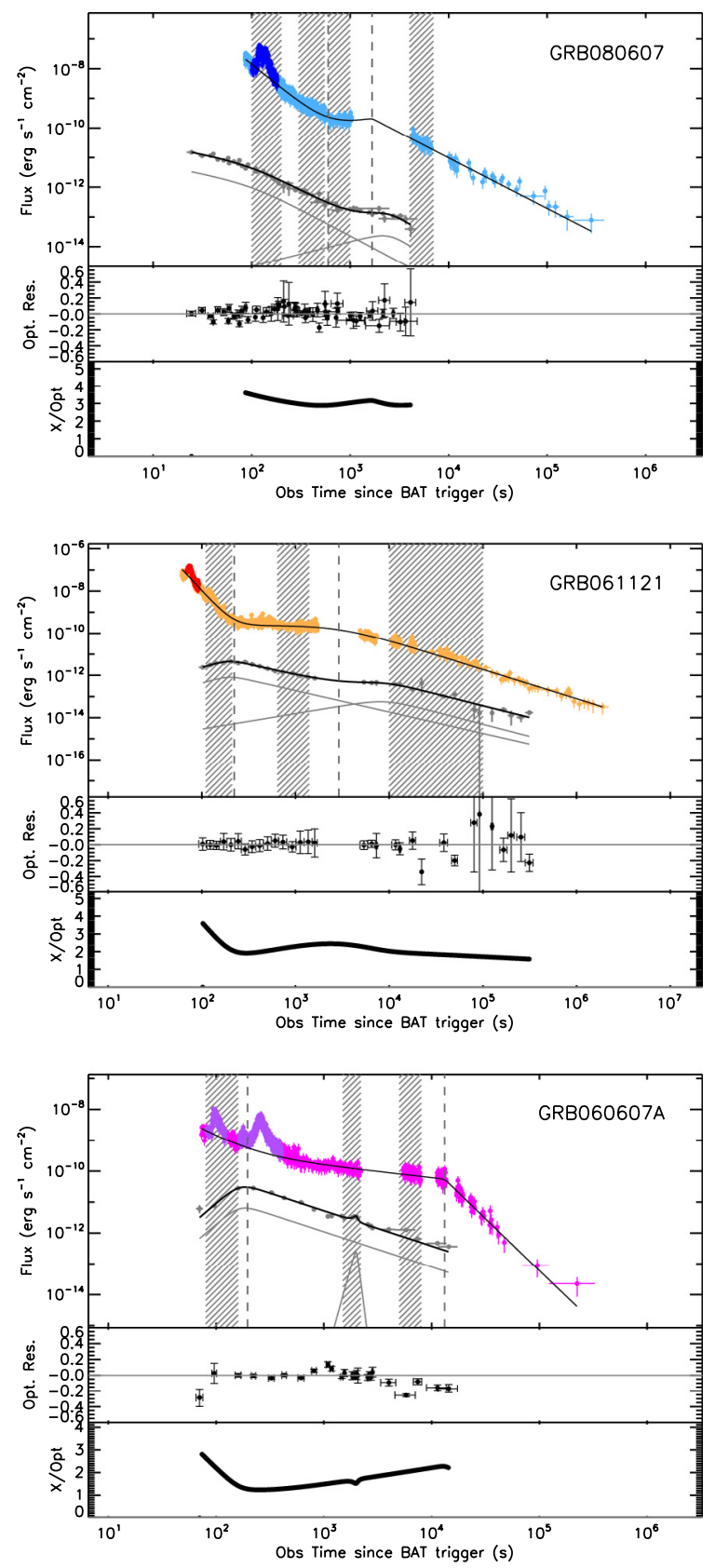

Fig. 14. Comparison between optical and X-ray LCs, examples of the GRBs in each group. Group A: GRB 080607 (blue/light blue). Group B: GRB 061121 (red/orange). Group C: GRB 060607A (purple/magenta). For each panel: Top. Colored points: X-ray data. The data in light color and bright color represent the continuum and the flaring portions, respectively, as calculated by M 13. Gray dashed lines: X-ray break times. Gray points: optical data. Black solid line: fit to the data. Gray solid lines: components of the fit function used to fit the optical data. Hashed gray boxes: SED time intervals. Middle. Ratio between the optical data and their fit function. Bottom. Ratio between the fit to the X-ray continuum and the optical LC. See Figs. C.1-C.9 for the other GRBs of our sample.

Thanks to our sample of LCs and SEDs, we were able to discuss the similarities and differences of the optical and X-ray emission by comparing their LCs (Sect. 4.1). In Sect. 4.2 we considered the forward-shock model and the closure relations (e.g. Sari et al. 1998; Zhang et al. 2006), and in Sect. 4.3 we presented the radio/optical/X-ray SED of GRB 071003, which is 
E. Zaninoni et al.: The gamma-ray burst optical light-curve zoo: comparison with the X-ray observations

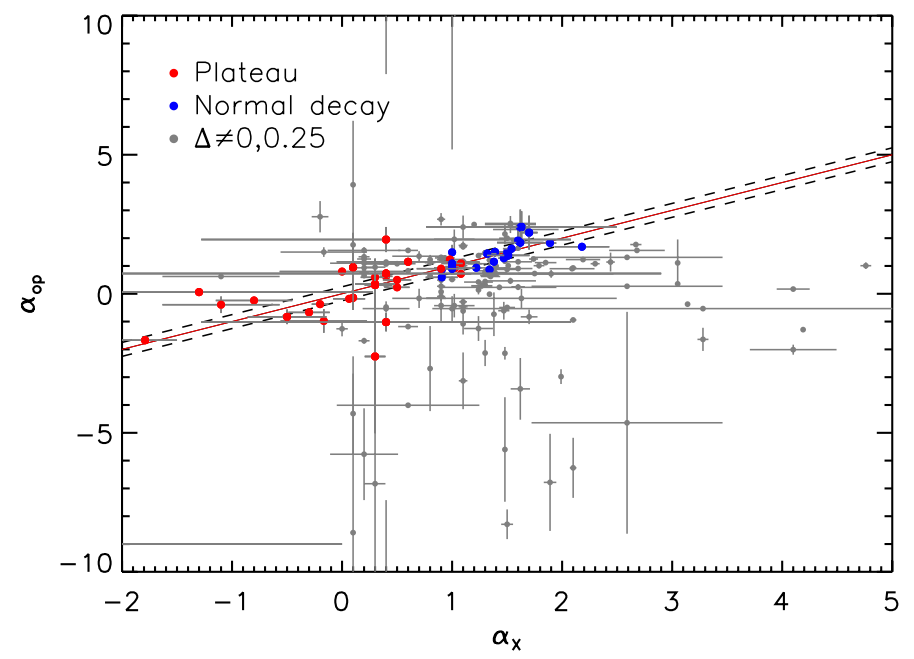

Fig. 15. Comparison between the X-ray LC slope $\left(\alpha_{X}\right)$ and the optical one $\left(\alpha_{\mathrm{op}}\right)$. Red (blue) dots: data for the plateau (normal decay) phase that agree with the $\Delta \alpha=0,1 / 4$ relation whitin the $1 \sigma$ errors. Gray dots: the data that do not follow the $\Delta \alpha=0,1 / 4$ relation. Red solid line: $\Delta \alpha=0$. Gray dashed lines: $\Delta \alpha= \pm 1 / 4$.

well fitted by the synchrotron spectrum. Finally, we investigated the role of the optical emission in the three-parameter correlation between $E_{\mathrm{X} \text {,iso }}-E_{\gamma, \text { iso }}-E_{\mathrm{pk}}$ (M 13, Bernardini et al. 2012b).

\subsection{LC phases}

\subsubsection{Steep-decay phase, the plateau, and the X-ray flares}

The steep-decay phase of the X-ray LCs is either the highlatitude emission after the end of the prompt emission (Kumar $\&$ Panaitescu 2000), or it may be part of the prompt emission itself, as proposed by Barniol Duran \& Kumar (2009) and Kumar et al. (2008), since the X-ray flux is smoothly connected to the $\gamma$-ray emission (Tagliaferri et al. 2005b; Goad et al. 2006) and is characterized by a strong hard-to-soft spectral evolution (Butler \& Kocevski 2007). In this phase most of the optical LCs rise $(42 \%)$ or have a complex behavior, with bumps, peaks, and plateaus (26\%), which makes their slopes different from the X-ray steep-decay slopes. The remaining 32\% decay during this phase, but of these there are only two cases whose optical LC slopes are similar to the X-ray slopes (e.g. GRB 080607, GRB 100901A). In some cases, there are X-ray flares superimposed on the steep decay, which are linked to the central engine activity (e.g. Zhang et al. 2006; Chincarini et al. 2010a).

The X-ray plateau is interpreted as an injection of energy into the forward shock (e.g. Zhang et al. 2006) and agrees with this prediction since there is no significant spectral evolution (Bernardini et al. 2012a). The source of the energy injection could be the power emitted by a spinning-down newly-born magnetar (Dai \& Lu 1998; Zhang \& Mészáros 2001; Corsi \& Mészáros 2009) that refreshes the forward shock (Dall'Osso et al. 2011) or the fall-back and accretion of the stellar envelope on the central black hole (Kumar et al. 2008). During this phase the optical LCs behave in different ways and $\sim 46 \%$ of them rise or show peaks or bumps.

From the comparison of the X-ray and optical LCs, we noted that there is a relation between the occurrence of the X-ray flares and the peak time of the optical LCs (Figs. 16-18). That is, when the flares are observed during the X-ray steep decay phase, the optical peak occurs early and before the beginning of the X-ray plateau, while if there are no flares or late-time flares, the optical peak occurs during the X-ray plateau.

The peak of the optical LC occurs during or at the end of the steep-decay phase if there are X-ray flares in this phase, as in GRB 080310, GRB 060512, GRB 061121, GRB 071031, GRB 081008, GRB 080928, GRB 060729, GRB 080607, GRB 060607A, and GRB 080810.

If the X-ray flares occur during the X-ray plateau, the optical LC peak or the end of the optical plateau occurs during the X-ray plateau: GRB 060124, GRB 050730, GRB 050820A, GRB 060210, GRB0 60904B, and GRB 060512.

In some cases it is difficult to evaluate this relation between the X-ray flares and the optical LCs. For GRB 060418 the computation of the X-ray break time is influenced by the presence of a very bright X-ray flare (e.g. Margutti et al. 2010b, for details). Accordingly, if we consider a break time $\sim 200 \mathrm{~s}$, the peak of the optical LC would be synchronous with the end of the steep-decay phase, and in this case it would be part of the group of GRBs with the break of the optical LC occurring during or at end of the steep decay. The GRB $060526 v$ filter data show a variability that corresponds with the X-ray flare, even if there is no true break. GRB 070318 has a Type 0 X-ray LC with a superimposed flare that temporally corresponds to the optical peak. At late time it shows another optical re-brightening that corresponds to a weak X-ray flux variation. GRB 070419A belongs to the $17 / 437$ Type $I I b$ GRBs with complete LCs (M 13). The end of the X-ray steep decay corresponds to the peak of the optical LC even though there are no flares during the steep decay. Unlike, the X-ray LC is not well sampled after the steep decay phase.

If there are no flares, the optical break occurs during the X-ray plateau. GRB 100418A and GRB 100901A simply follow the trend of the X-ray data. The LC of GRB 060614 is similar to that of GRB 100418A and GRB 100901A, but there are no data during the X-ray steep decay. For GRB 050319, GRB 081203A, GRB 060605, GRB 060906, GRB 070802, and GRB 071025 some data are lacking during the plateau and the steep decay is not well sampled or is of short duration. GRB 071112C and GRB 080413B have Type Ia X-ray LC (M 13) and so there is no steep decay phase. GRB 081029 has no X-ray data before the optical peak. For GRB 091127 we have only late-time data $\left(t_{\text {start,X }} \sim 5 \times 10^{3} \mathrm{~s}\right)$. The optical peak of GRB 051109A corresponds to the end time of the $\mathrm{X}$-ray plateau, but there are no X-ray data during the plateau. GRB 050408, whose observations started $2000 \mathrm{~s}$ after the trigger, has a Type 0 LC and shows an optical break at about $2 \times 10^{4} \mathrm{~s}$, even though the X-ray LC does not show flux variations.

For GRB 050904, GRB 061007, GRB 080603A, GRB 050525A, GRB 080710, GRB 090313, GRB 090926A, GRB 070125, GRB 060729, GRB 080330, GRB 071003, GRB 071010A, GRB 070208, GRB 070411, and GRB 090426 the X-ray data are very poor.

The relation between the $\mathrm{X}$-flares and the optical peaks and plateaus is displayed also in GRB 070110, GRB 080319A, GRB 081126, GRB 090812, and GRB 100906A studied by Li et al. (2012) and Liang et al. (2012).

\subsubsection{Normal decay phase}

The X-ray normal decay is present in most of the X-ray LCs of our sample (63/68). Seventy-seven per cent of the optical LCs decay during the X-ray normal phase, but only $62 \%$ have a similar slope. In addition, only a few cases follow the closure relations. 
A\&A 557, A12 (2013)
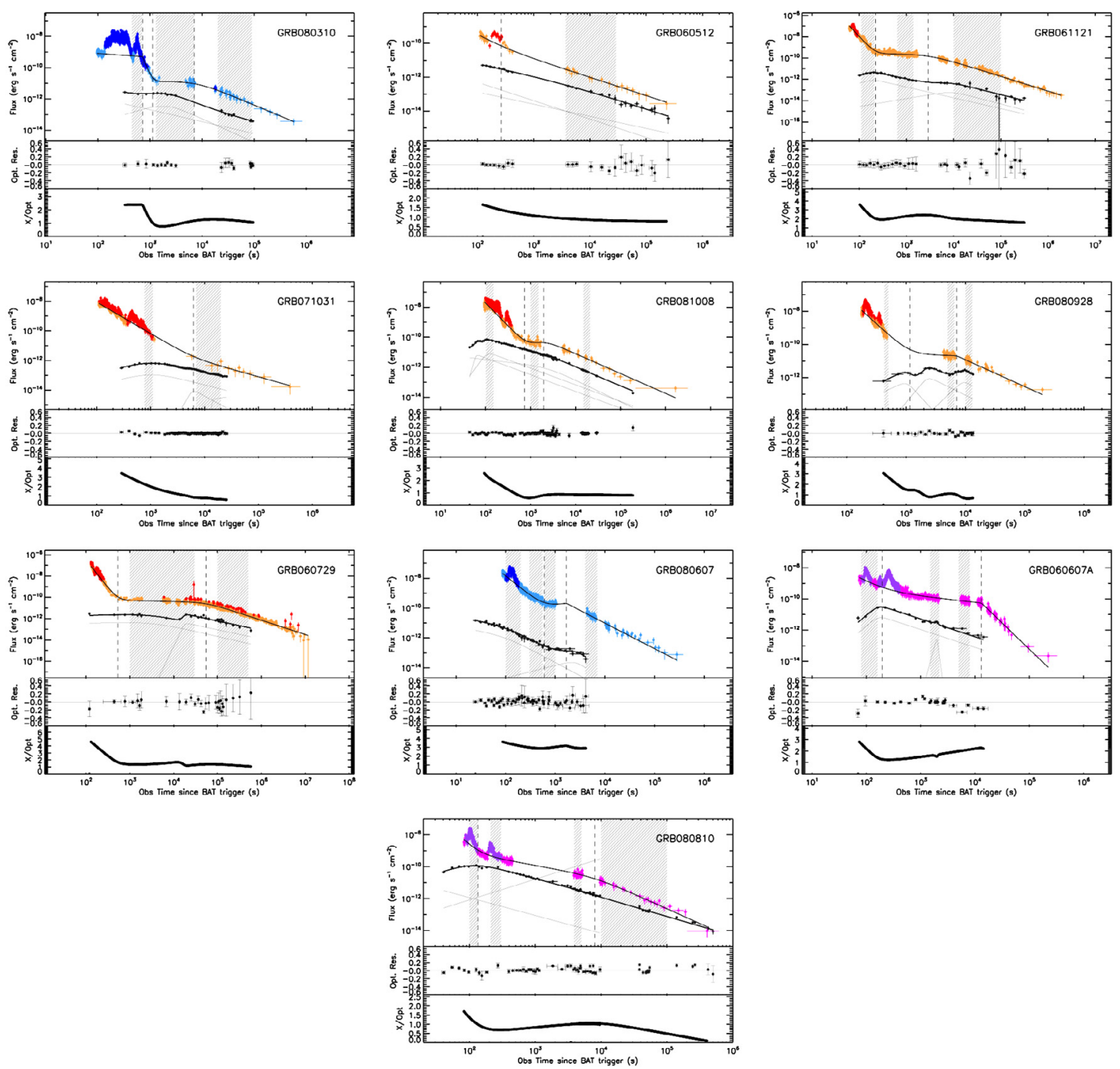

Fig. 16. GRBs with X-ray flares during the steep-decay phase and the optical peak during or at the end of the X-ray steep decay. Color code as in Fig. 14.
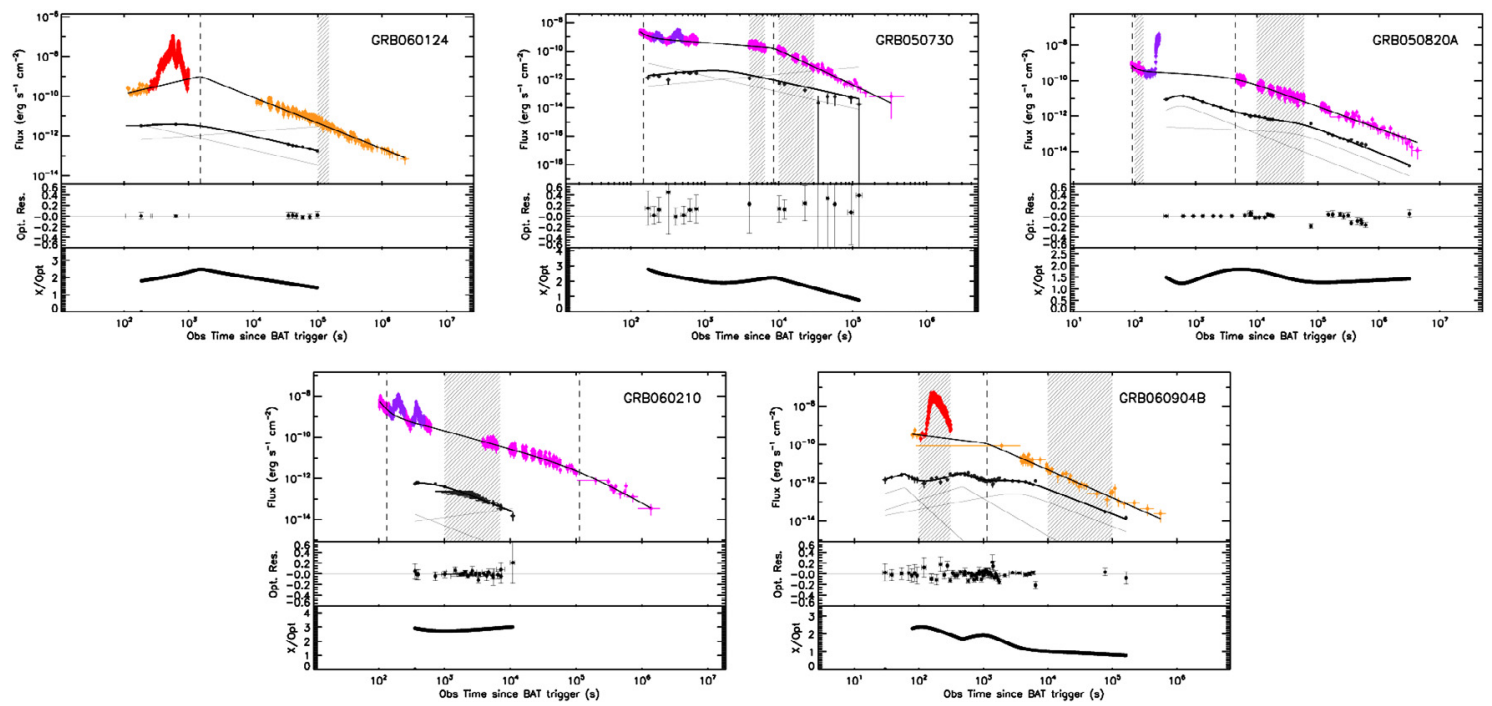

Fig. 17. GRBs with X-ray flares during the plateau and the optical peak during or at the end of the X-ray plateau. Color code as in Fig. 14. 
E. Zaninoni et al.: The gamma-ray burst optical light-curve zoo: comparison with the X-ray observations
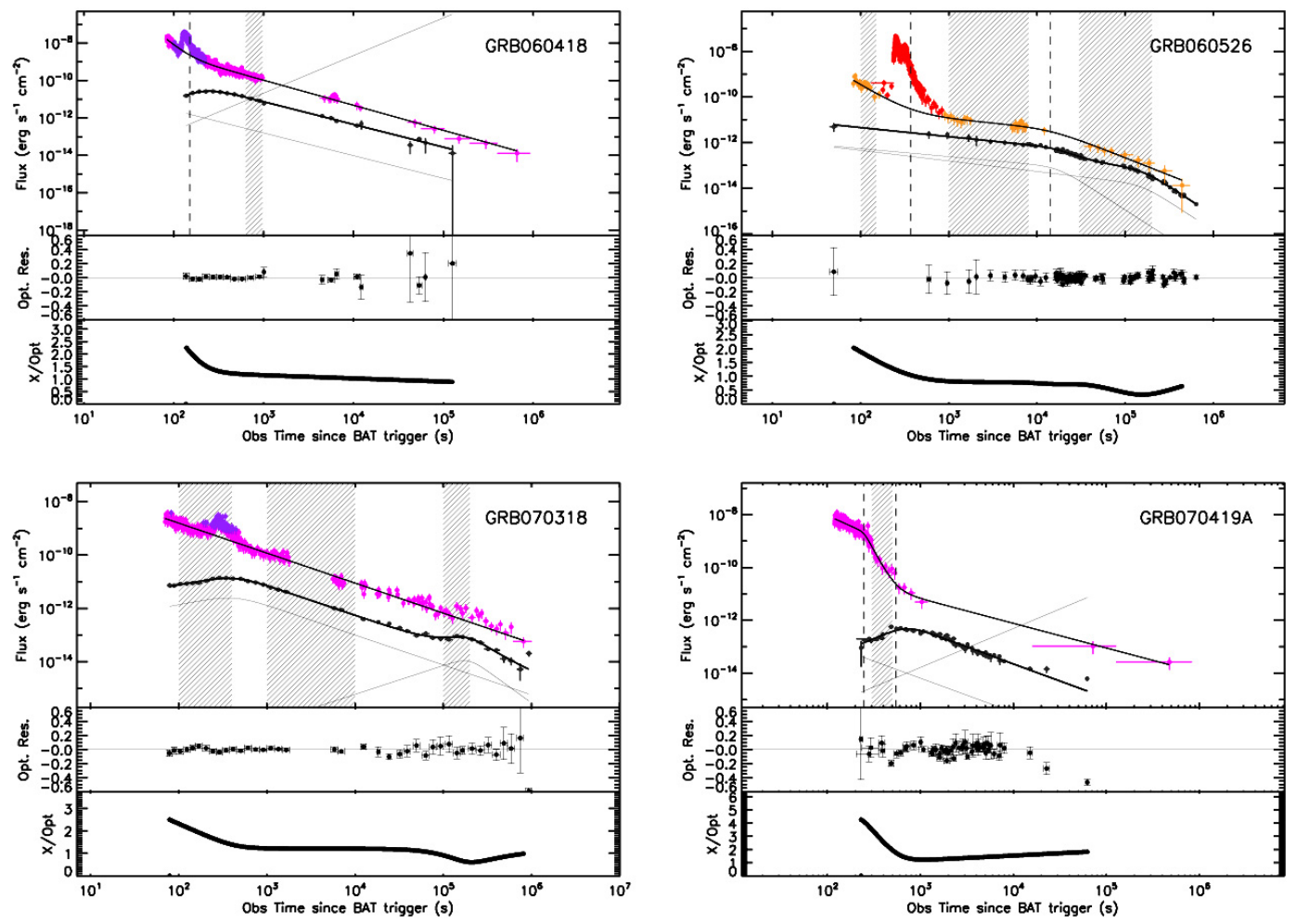

Fig. 18. Particular cases. Color code as in Fig. 14.

Twenty-three per cent of the optical LCs contemporaneous to the X-ray normal decay have a variable shape with bumps and late-time re-brightenings. Small bumps could represent late central engine activity (e.g. Li et al. 2012) or a change in the circumburst density (Lazzati et al. 2002), while the late-time rebrightenings could be a result of the jet structure (e.g. Liang et al. 2012).

The normal decay phase of the X-ray LC and the contemporaneous optical emission can be considered as afterglow emission, even though the central engine activity is revealed by the late-time X-ray flares (e.g. Bernardini et al. 2011) and optical bumps and re-brightenings.

\subsection{Closure relations}

The standard fireball model predicts a link between the characteristic quantities of the spectrum and the LC of a GRB afterglow (Sari et al. 1998). We compared the temporal decay and spectral indices derived from our analysis of the X-ray and optical LCs in the plateau and normal decay phases with these predictions (see also Zhang et al. 2006, their Table 2). We restricted our analysis to the slow-cooling regime, with either a constant ISM or a wind medium, including the possibility of energy injection ${ }^{23}$.

\footnotetext{
${ }^{23}$ In particular, we considered the following cases: a) $\alpha_{\mathrm{op}}-\alpha_{\mathrm{X}}=0$ : if there is no energy injection, $\alpha=(3 \beta-1) / 2$; if there is energy injection, $\alpha=(q-2+(2+q) \beta) / 2$. b) $\alpha_{\mathrm{op}}-\alpha_{\mathrm{X}}=-1 / 4$ corresponds to a constant density medium in the slow cooling regime with no energy injection, so $\alpha_{\text {op }}=3 \beta_{1} / 2$ and $\alpha_{\mathrm{X}}=\left(3 \beta_{2}-1\right) / 2$. c) $\alpha_{\mathrm{op}}-\alpha_{\mathrm{X}}=+1 / 4$ corresponds to a wind medium in the slow cooling regime, so $\alpha_{\text {op }}=\left(3 \beta_{1}+1\right) / 2$ and $\alpha_{X}=$ $\left(3 \beta_{2}-1\right) / 2$. d) $\alpha_{\text {op }}-\alpha_{\mathrm{X}}=(q-2) / 4$ corresponds to a constant density medium in the slow cooling regime with energy injection, so $\alpha_{\mathrm{op}}=$ $(q-1)+\left(\beta_{1} / 2\right)(2+q)$ and $\alpha_{\mathrm{X}}=((q-2)+\beta(2+q)) / 2$. e) $\alpha_{\mathrm{op}}-\alpha_{\mathrm{X}}=(2-q) / 4$ corresponds to a wind medium in the slow cooling regime with energy injection, so $\alpha_{\mathrm{op}}=\left(q+\beta_{2}(2+q) \beta\right) / 2$ and $\alpha_{\mathrm{X}}=((q-2)+\beta(2+q)) / 2$. In
}

Overall, we find that $4 \%$ of the GRBs of our sample are consistent with the closure relations within $2 \sigma$ during the plateau phase (GRBs in group B) and 12\% during the normal decay (4\% in group A and $8 \%$ in group B). We define as $\alpha$ the value of the LC slope calculated fitting the LCs and $\tilde{\alpha}$ the LC slope expected following the closure relations, determined using the spectral indices computed with the SEDs. For Group A (defined in Sect. 3.4), the closure relations are valid in some GRBs only for the normal decay phase:

- GRB 080607: its optical/X-ray SED, extracted during the normal decay phase, is fitted with a broken power-law function and follows the relation $\alpha_{\mathrm{op}}-\alpha_{\mathrm{X}} \sim 1 / 4$ because $\alpha_{\text {op }}=2.20 \pm 0.61$ and $\alpha_{\mathrm{X}}=1.70 \pm 0.07$ (constant-density medium in the slow cooling regime without energy injection). Therefore $\tilde{\alpha}_{\text {op }}=\left(3 \beta_{1}+1\right) / 2$ and $\tilde{\alpha}_{\mathrm{X}}=\left(3 \beta_{2}-1\right) / 2$. From the spectrum $\beta_{\mathrm{op}}=0.80 \pm 0.10$ and $\beta_{\mathrm{X}}=1.30 \pm 0.10$, then the expected slopes are $\tilde{\alpha}_{\text {op }}=1.70 \pm 0.15$ and $\tilde{\alpha}_{X}=1.45 \pm 0.15$.

- GRB 050416A: during the normal decay, the best fit of the SED is a broken power-law. $\alpha_{\mathrm{op}}=0.86 \pm 0.15$ and $\alpha_{\mathrm{X}}=$ $0.90 \pm 0.04$ and $\alpha_{\mathrm{op}}-\alpha_{\mathrm{X}} \sim \pm 1 / 4$ within $2 \sigma$. This agrees with a slow cooling regime without energy injection in a constant density medium: $\tilde{\alpha}_{\mathrm{op}}=3 \beta_{\mathrm{op}} / 2=0.78 \pm 0.23$ and $\tilde{\alpha}_{\mathrm{X}}=$ $(3 \beta-1) / 2=1.03 \pm 0.21$.

- GRB 100418A: the best-fitting function of the SED is a single power-law. $\alpha_{\mathrm{op}}=1.38 \pm 0.07, \alpha_{\mathrm{X}}=1.51 \pm 0.19$ and $\beta_{\mathrm{op}, \mathrm{X}}=1.19 \pm 0.02$. If there is no energy injection, ISM, or wind medium, for $v_{\mathrm{c}}<v_{\mathrm{op}}<v_{\mathrm{X}}$ the expected slopes are $\tilde{\alpha}_{\text {op }}=\tilde{\alpha}_{\mathrm{X}}=\left(3 \beta_{\mathrm{op}, \mathrm{X}}-1\right) / 2=1.39 \pm 0.03$ in agreement, within errors, with our data.

For Group B, for the normal decay:

- GRB 071003: the X-ray LC has a simple power-law shape, whereas the optical LC has an initial shallow decay followed

the energy injection case, we considered three values of $q, 0,0.3,0.5$, which depend on the strength of the injection. 
by a normal decay. For this last part, $\alpha_{\mathrm{op}}=1.91 \pm 0.36$ and $\alpha_{\mathrm{X}}=1.60 \pm 0.06$. The best fit of the SED is a simple power-law $\left(\beta_{\mathrm{op}, \mathrm{X}}=1.28 \pm 0.05\right)$, so the expected $\tilde{\alpha}=$ $1.42 \pm 0.08$ for no energy injection, ISM, or wind medium, and $v_{\mathrm{c}}<v_{\mathrm{op}}<v_{\mathrm{X}}$. This agrees with the X-ray and the optical slopes within $2 \sigma$.

- GRB 080413B: for the normal decay phase, $\alpha_{\mathrm{op}}=1.84 \pm$ 0.08 and $\alpha_{\mathrm{X}}=1.62 \pm 0.21$. The best fit of the SED is a broken power-law $\left(v_{\mathrm{op}}<v_{\mathrm{c}}<v_{\mathrm{X}}\right)$. The expected X-ray slope is $\tilde{\alpha}_{\mathrm{X}}=1.36 \pm 0.36$ for the case without energy injection and $\tilde{\alpha}_{\text {op }}=1.11 \pm 0.36$ for the slow cooling regime and $\tilde{\alpha}_{\text {op }}=$ $1.61 \pm 0.36$ for the fast cooling regime. Therefore the normal decay observations agrees with an ISM medium, no energy injection and slow $(2 \sigma)$ or fast $(1 \sigma)$ cooling regime.

- GRB 080913: the X-ray LC is a simple power-law with $\alpha_{\mathrm{X}}=1.00 \pm 0.06$. The optical LC shows a late-time re-brightning, but at the beginning, between 600-1200 s after the trigger, $\alpha_{\mathrm{op}}=0.98 \pm 0.04$. The best fit of the SED is a simple power-law and follows the closure relation for the ISM or wind medium in the slow cooling regime and $v_{\mathrm{m}}<v_{\mathrm{op}}<v_{\mathrm{X}}<v_{\mathrm{c}}$; in fact $\tilde{\alpha}_{\mathrm{op}}=\tilde{\alpha}_{\mathrm{X}}=0.98 \pm 0.06$ in the ISM case and $\tilde{\alpha}_{\text {op }}=\tilde{\alpha}_{X}=1.06 \pm 0.05$ in the wind case.

- GRB080928: for the normal decay, $\alpha_{\text {op }}=2.38 \pm 0.65$ and $\alpha_{\mathrm{X}}=1.62 \pm 0.09$. The best-fit function is a power-law, hence there is one spectral index, $\beta_{\mathrm{op}, \mathrm{X}}=1.15 \pm 0.02$. The expected slope both for the X-ray and the optical LC is $\tilde{\alpha}=1.69 \pm$ 0.02 for the case of slow cooling, energy injection, and wind medium, and $v_{\mathrm{m}}<v_{\mathrm{op}}<v_{\mathrm{X}}<v_{\mathrm{c}}$. $\tilde{\alpha}$ is consistent with $\alpha_{\mathrm{X}}$ $(1 \sigma)$ and $\alpha_{\text {op }}(2 \sigma)$.

- GRB 081008: for the X-ray normal decay $\alpha_{\mathrm{X}}=1.32 \pm 0.08$ and the corresponding optical slope is $\alpha_{\mathrm{op}}=1.44 \pm 0.13$. The spectral index is $\beta_{\mathrm{op}, \mathrm{X}}=0.93 \pm 0.01$. Without energy injection, ISM, and $v_{\mathrm{m}}<v_{\mathrm{op}}<v_{\mathrm{X}}<v_{\mathrm{c}}$, the expected slope is $\tilde{\alpha}=1.39 \pm 0.01$, which agrees with the optical and X-ray slopes of this GRB.

For Group B, for the plateau:

- GRB 060502A: $\alpha_{\text {op }}=0.50 \pm 0.05$ and $\alpha_{\mathrm{X}}=0.45 \pm 0.13$. The best fit of the SED is a broken power-law, hence $\beta_{\mathrm{op}}=$ $0.51 \pm 0.12$ and $\beta_{\mathrm{X}}=1.01 \pm 0.12$. For the case of energy injection, ISM, and slow cooling regime, we obtain $\tilde{\alpha}_{\text {op }}=$ $0.14 \pm 0.15$ and $\tilde{\alpha}_{\mathrm{X}}=0.51 \pm 0.16$. These values agree with the optical $(3 \sigma)$ and the X-ray slope $(1 \sigma)$.

- GRB 060512: $\alpha_{\mathrm{op}}=1.09 \pm 0.15$ and $\alpha_{\mathrm{X}}=1.08 \pm 0.09$. The best fit of the SED is a single power-law $\left(\beta_{\mathrm{op}, \mathrm{X}}=\right.$ $1.24 \pm 0.05)$. Therefore we obtain $\tilde{\alpha}=1.05 \pm 0.06$, which is consistent with the optical and X-ray slopes. This result was obtained considering energy injection, ISM, slow cooling regime, and $v_{\mathrm{m}}<v_{\mathrm{op}}<v_{\mathrm{X}}<v_{\mathrm{c}}$.

- GRB 071031: $\alpha_{\text {op }}=0.93 \pm 0.03$ and $\alpha_{\mathrm{X}}=1.02 \pm 0.18$. The best fit of the SED is a single power-law with $\beta_{\mathrm{op}, \mathrm{X}}=$ $0.99 \pm 0.01$. The expected LC slope is $\tilde{\alpha}=0.99 \pm 0.02$ for the case without energy injection and $v_{\mathrm{c}}<v_{\mathrm{op}}<v_{\mathrm{X}}$. This value agrees with the optical LC slope $(2 \sigma)$ and the X-ray slope $(1 \sigma)$.

We found no GRB that followed the closure relations for all phases of the LC, indeed, all GRBs of our sample are inconsistent with the closure relations at least in one of the phases of their LC.

The closure relations correspond to the broadband spectrum and LC of synchrotron radiation from a power-law distribution of electrons in an adiabatically expanding relativistic shock, as expected in the standard afterglow theory. The inconsistency with the data implies that this model, at least in its simplest formulation, is unlikely to produce the observed afterglow emission. Since optical and X-ray observations are well fitted with a synchrotron spectrum, a more complex hydrodynamic evolution of the outflow must be considered, or alternatively, a direct influence from central engine.

\subsection{Broadband SEDs}

From the GRB sample with radio data presented by Chandra \& Frail (2012) we selected two GRBs (GRB 071003 and GRB 090313) whith radio observations contemporaneous with the optical/X-ray data presented here to test the broadband behavior of the SEDs.

For GRB 071003 we calculated a set of illustrative radio/optical/X-ray SEDs starting from different values of the cooling frequency $\left(v_{\mathrm{c}}\right)$ and the maximum flux $\left(F_{v, \max }\right)$, as presented by Sari et al. (1998) for the slow cooling regime ${ }^{24}$. The optical/X-ray SED was fitted with a simple power-law, so the cooling frequency is probably below the optical band. In this way we found a possible set of data that is consistent with the radio/optical/X-ray SED is: $v_{\mathrm{a}}=1.2 \times 10^{11} \mathrm{~Hz}, v_{\mathrm{m}}=$ $3.08 \times 10^{11} \mathrm{~Hz}, v_{\mathrm{c}}=9.45 \times 10^{12} \mathrm{~Hz}$ and $F_{v, \max }=198 \mathrm{mJy}$ (Fig. 19). The total energy, calculated in the frequency interval $v=10^{7}-10^{19} \mathrm{~Hz}$, is $2.72 \times 10^{52} \mathrm{erg}$. For the different parts of the spectrum, the energy is $E_{10^{7} \mathrm{~Hz}-v_{\mathrm{a}}}=1.25 \times 10^{50}$ erg, $E_{v_{\mathrm{a}}-v_{\mathrm{m}}}=9.56 \times 10^{50} \mathrm{erg}, E_{v_{\mathrm{m}}-v_{\mathrm{c}}}=8.59 \times 10^{51} \mathrm{erg}$, and $E_{\nu_{\mathrm{c}}-10^{19} \mathrm{~Hz}}=1.76 \times 10^{52} \mathrm{erg}$.

This example shows that the synchrotron model can adequately fit the GRB spectral properties at late time.

For GRB 090313 we know the cooling frequency, which is the break frequency calculated by fitting the optical/X-ray SED with a broken power-law. At this time the data are unlikely to be influenced by the host galaxy, and we cannot reconstruct the radio/optical/X-ray SED, as shown in Fig. 19.

\subsection{Three-parameter correlation}

In M 13 we found a three-parameter correlation, which we discussed in Bernardini et al. (2012b). It involves the isotropic energy emitted in the rest-frame $1-10^{4} \mathrm{keV}$ energy band during the prompt emission $\left(E_{\gamma, \text { iso }}\right)$, the peak of the prompt emission energy spectrum $\left(E_{\mathrm{pk}}\right)$ and the X-ray energy emitted in the $0.3-30 \mathrm{keV}$ observed energy band $\left(E_{\mathrm{X}, \text { iso }}\right)$, which is integrated over the observed duration of each LC. The X-ray energy was calculated in a specific energy band, and we did not extrapolate the spectrum to lower energies because we did not know the behavior of the spectrum at those energies. In this work we have presented the GRB spectra covering the energy band from the infrared to $\mathrm{X}$-rays and calculated the models that fit the data better. Using these models, for every GRB we calculated the ratio between

\footnotetext{
${ }^{24}$ For an adiabatic evolution, the equations that describe the emitted radiation are (Sari et al. 1998; Granot et al. 2000): $F_{v}=$ $\left(v_{\mathrm{a}} / v_{\mathrm{m}}\right)^{1 / 3}\left(v / v_{\mathrm{a}}\right)^{2} F_{v, \text { max }}$ for $v<v_{\mathrm{a}} ; F_{v}=\left(v / v_{\mathrm{m}}\right)^{1 / 3} F_{v, \max }$ for $v_{\mathrm{m}}>v ; F_{v}=$ $\left(v / v_{\mathrm{m}}\right)^{-(p-1) / 2} F_{v, \text { max }}$ for $v_{\mathrm{c}}>v>v_{\mathrm{m}} ; F_{v}=\left(v_{\mathrm{c}} / v_{\mathrm{m}}\right)^{-(p-1) / 2}\left(v / v_{\mathrm{c}}\right)^{-p / 2} F_{v, \text { max }}$ for $v>v_{\mathrm{c}}$. The absorption frequency is $v_{\mathrm{a}}=0.247\left(4.24 \times 10^{9}\right)((p+$ $2) /(3 p+2))^{3 / 5}\left((p-1)^{8 / 5} /(p-2)\right) \epsilon_{\mathrm{e}}^{-1} \epsilon_{\mathrm{B}}^{1 / 5} E_{52}^{1 / 5} n_{1}^{3 / 5} \mathrm{~Hz}$; the synchrotron frequency $v_{\mathrm{m}}=\left(5.7 \times 10^{14}\right) \epsilon_{\mathrm{B}}^{1 / 2} \epsilon_{\mathrm{e}}^{2} E_{52}^{1 / 2} t_{\mathrm{d}}^{-3 / 2} \mathrm{~Hz}$; the cooling frequency $v_{\mathrm{c}}=\left(2.7 \times 10^{12}\right) \epsilon_{\mathrm{B}}^{-3 / 2} E_{52}^{-1 / 2} n_{1}^{-1} t_{\mathrm{d}}^{-1 / 2} \mathrm{~Hz}$; the maximum flux, which is the flux at $v_{\mathrm{m}}, F_{v, \text { max }}=\left(1.1 \times 10^{5}\right) \epsilon_{\mathrm{B}}^{1 / 2} E_{52} n_{1}^{1 / 2} D_{28}^{-2} \mu \mathrm{Jy} . t_{\mathrm{d}}$ is the time in days, $E_{52}=E / 10^{52} \mathrm{ergs}$, the density $n_{1}$ in $\mathrm{cm}^{-3}$, the distance $D_{28}=D / 10^{28} \mathrm{~cm}$.
} 

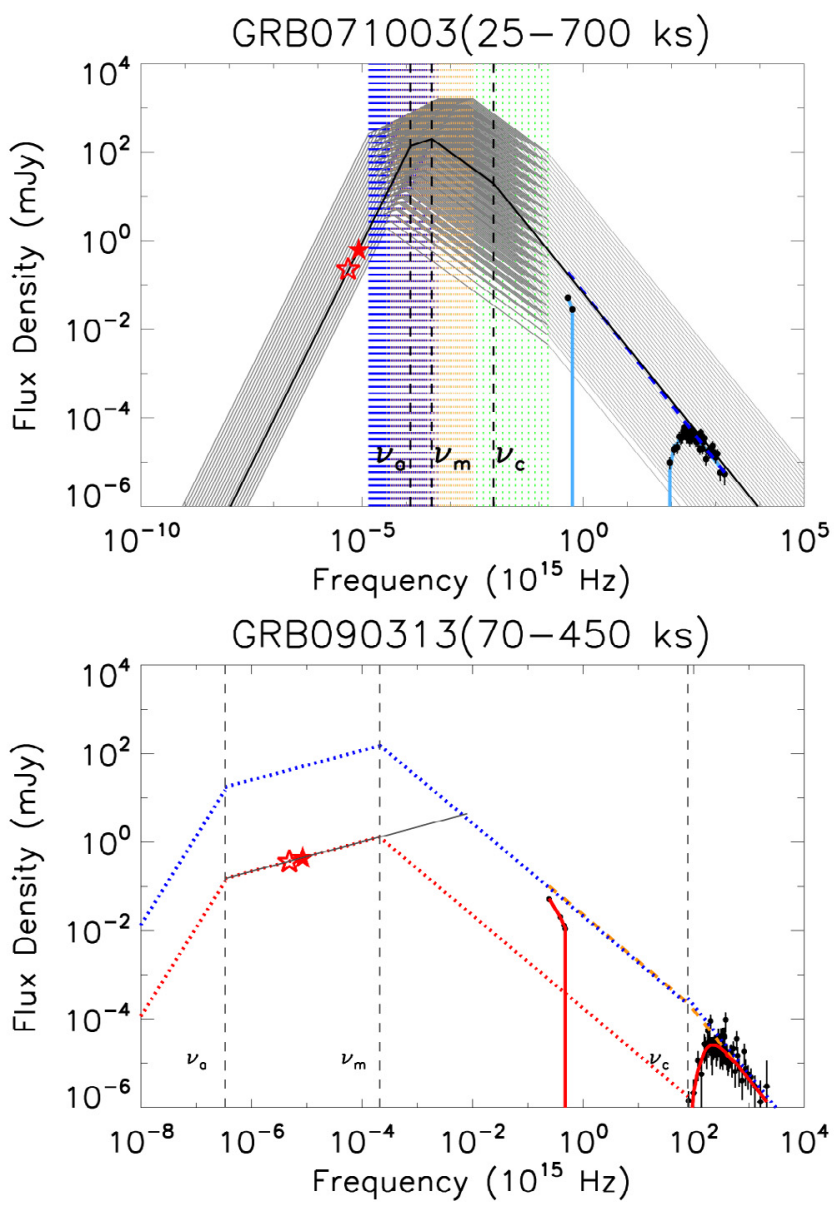

Fig. 19. Radio/optical/X-ray SEDs. Filled (empty) red star: radio data (upper limit). Black dots: optical and X-ray data. Upper panel: GRB 071003. Light blue solid line: optical/X-ray SED. Blue dashed line: power-law that fits the data. Black solid line: radio/optical/X-ray SED fit function. Black dashed line: the absorption frequency $\left(v_{a}\right)$, the synchrotron frequency $\left(v_{\mathrm{m}}\right)$, and the cooling frequency $\left(v_{\mathrm{c}}\right)$. Gray lines: radio/optical/X-ray SED tests with relative absorption frequencies (blue dashed lines), synchrotron frequencies (orange dashed lines), and cooling frequencies (green dashed lines). Lower panel: GRB 090313. Red solid line: the optical/X-ray SED fit function. Orange dashed line: broken power-law that fits the data. Blue dotted line: radio/optical/X-ray SED calculated considering $\nu_{\mathrm{BR}}=v_{c}$. Red dotted line: radio/optical/X-ray SED calculated using the same characteristic frequencies as the red dotted line, but considering the normalization for the radio data. Gray dashed line: the absorption frequency $\left(v_{a}\right)$, the synchrotron frequency $\left(v_{\mathrm{m}}\right)$, and the cooling frequency $\left(v_{\mathrm{c}}\right)$.

the X-ray energy band $(0.3-30 \mathrm{keV})$ and the total energy emitted from the IR to X-rays: $\sim 70 \%$ of the total energy is radiated in the X-rays. The mean value of the IR-optical-UV energy ${ }^{25}$ is $\lesssim 10 \%$.

The correlation is not quantitatively modified by including the optical energy. However, since the origin of the correlation is still unknown, it is not clear if including the optical energy is conceptually necessary or not.

\section{Conclusions}

We performed a systematic analysis of a large sample of well-monitored optical LCs (68 GRBs) obtained with different instruments and compared them with the X-ray emission. Since

\footnotetext{
${ }^{25}$ We considered the $0.5-6 \mathrm{keV}$ band.
}

the GRBs of this sample have known redshift, we considered their rest frame properties. From this large sample of observations:

- we fitted the optical LCs collected from different instruments and the optical/X-ray SEDs at different times for every GRB;

- we studied the distribution of the parameters computed from the SEDs, which yield information on the medium and the spectrum. We found that a) there is a slight softening of the optical/X-ray spectrum with time; b) the gas-to-dust ratios $\left(N H / A_{\mathrm{V}}\right)$ of GRBs are higher than the values calculated for the MW, the LMC, and SMC assuming subsolar abundances (e.g. Schady et al. 2010, 2012); and c) the break frequencies are spread between the optical and X-ray bands;

- for a given rest frame time range (920-12000 s), the optical flux in the $R$ band is $\sim 2$ orders of magnitude brighter than the X-ray flux in the $1 \mathrm{keV}$ band;

- there is a correlation between the energy of the peak of the optical LCs and $E_{\gamma, \text { iso }}$ and $E_{\gamma}^{15-150}$, confirming the result of Panaitescu \& Vestrand (2011). The optical plateau end luminosity and rest frame time are correlated, and similarly, the peak luminosity and the peak times are correlated (e.g. Panaitescu \& Vestrand 2011);

- the optical LCs have a complex shape, with initial plateaus and peaks, bumps, and late-time re-brightenings. With time, the complexity of the LCs decreases and more and more of them decay;

- only for 13 GRBs of our sample (Group A), all LC segments follow $\alpha_{\mathrm{op}}-\alpha_{\mathrm{X}}=0, \pm 1 / 4^{26}$, in the other cases the X-ray and optical LCs show a different behavior;

- the onset of the forward shock observed in the optical LCs could be linked to the presence of the X-ray flares. Indeed, when there are X-ray flares during the steep decay, the optical LC peak or the end of the initial plateau occurs during or just at the end of the X-ray steep-decay phase, while if there are no flares or the flares take place during the X-ray plateau, the optical peak or plateau end occurs during the X-ray plateau;

- the forward shock model cannot explain all features of the optical and X-ray LCs, such as bumps, flares, rebrightenings, steep decays, and plateaus, either at early or late time;

- the contribution of the optical energy to the three-parameter correlation (M 13, Bernardini et al. 2012b) is low, less than $10 \%$ of the total energy emitted from the IR and X-rays. Therefore the correlation is not quantitatively modified by including the optical energy. However, since the origin of the correlation is still unknown, it is not clear whether including the optical energy is conceptually necessary or not.

From this study it clearly emerges that the complex shapes of the optical and X-ray LCs cannot be explained simply by the forward shock, although we can confirm that the synchrotron is a viable emission mechanism for GRBs at late times. Moreover, we showed the importance of a systematic analysis of the GRB multi-wavelength observations. To improve the knowledge of the physics of GRBs and their origin, very fast detectors and multi-wavelength observations are needed. In particular, we need an optical follow-up starting at the latest a few seconds after the trigger to collect homogeneous spectroscopic and photometric data (e.g. Chincarini et al. 2010b).

${ }^{26}$ We did not consider the steep decay, since during this phase the emission from the two bands clearly comes from different mechanisms or emitting regions. 
Acknowledgements. We thank the anonymous referee for the helpful comments that have improved this paper. E.Z. thanks Daniele Malesani for the useful discussions, suggestions and support during the preparation of the paper; Paolo D'Avanzo and Andrea Melandri for the useful advices; Thomas Krüler for sharing the data of GRB 070802 and Fang Yuang for the data of GRB 081008; Craig B. Markwardt for the help with the MPFIT routine. This research has made use of the XRT Data Analysis Software (XRTDAS) developed under the responsibility of the ASI Science Data Center (ASDC), Italy. This work was supported by ASI grant Swift I/011/07/0 and in part by I/004/11/0, by Ministero degli Affari Esteri and the University of Milano - Bicocca.

\section{Appendix A: Optical data conversion factors}

In the literature data are generally given in different photometric systems and based on the Vega or $\mathrm{AB}$ magnitude convention. The Vega system is defined as the system for which the magnitude of the Vega star is zero: $m-0=-2.5 \log f_{\lambda}+2.5 \log f_{\lambda \text {, Vega }}$, with $f_{\lambda}$ and $f_{\lambda \text {,Vega }}$ in erg $\mathrm{cm}^{-2} \mathrm{~s}^{-1} \AA^{-1}$. In this case, the conversion formula used is $f_{\lambda}=f_{\lambda \text {, vega }} 10^{-0.4} \mathrm{~m}$. The relation between flux density in frequency and wavelength units is $f_{v}=\lambda^{2} f_{\lambda} / c$, with $c$ the speed of light and $\lambda$ the central wavelength of the considered filter. For the AB system the reference spectrum is flat in units of frequency density ( $\mathrm{erg} \mathrm{cm}^{-2} \mathrm{~s}^{-1} \mathrm{~Hz}^{-1}$ ) and the system is defined by $m_{\mathrm{V}}^{\mathrm{Vega}} \equiv m_{\mathrm{V}}^{\mathrm{AB}} \equiv 0$ in the visual band; this occurs at 3631 Jy. So $m-m_{\mathrm{AB}, 0}=-2.5 \log f_{v}-2.5 \log \left(3631 \times 10^{-23}\right)=$ $-2.5 \log f_{v}-(48.585 \pm 0.005)$, with $f_{v}$ in $\mathrm{erg} \mathrm{cm}^{-2} \mathrm{~s}^{-1} \mathrm{~Hz}^{-1}$. The conversion formula is $f_{v}=10^{-0.4(m+48.585)}$. In various articles, the photometric system is not always specified, which leads to mistakes in converting from magnitude to flux. For UVOT data the calibration has been provided by the UVOT team, and we used their conversion factors (Poole et al. 2008). Some errors might occur when the photometric system is not specified, especially for the Gunn system or the standard Johnson system; for example, for the $R$ filter, the Gunn conversion factor is twice as much the standard system conversion factor. On the other hand, the IR filters used in literature are very similar. In this case, the choice of the photometric system does not noticeably influence the following analysis, and we preferred to choose only standard systems listed in Table C.7.

\section{Appendix B: Description of the GRBs in the three groups}

Group A. All pairs of optical/X-ray slopes of the same GRB satisfy the relation $\alpha=0, \pm 1 / 4$. The $\mathrm{X}$-ray plateau and normal decay phase follows the optical slopes for seven GRBs: GRB 080607, GRB 100901A, GRB 050416A, GRB 080310, GRB 080319B, GRB 100418A, and GRB 050824.

For six GRBs we can compare the optical and X-ray LCs only during the X-ray plateau or X-ray normal decay, because of the poor data. GRB 060912A, GRB 061007, and GRB 080913 have a Type 0 complete X-ray LC. GRB 060912A has a simple power-law optical LC. The GRB 061007 optical LC rises at early time, where there are no X-ray observations, and then follows the normal decay of the X-ray LC. Finally, the GRB 080913 optical LC traces the X-ray LC during the first and the last part for its optical LC. Unfortunately, there are no optical observations between $10^{4}$ and $10^{5} \mathrm{~s}$. For GRB $080603 \mathrm{~A}$ there are no observations before $10^{4} \mathrm{~s}$; GRB $050904\left(\alpha_{\mathrm{op}}-\alpha_{\mathrm{X}}=0.25\right)$ has a Type $I b$ $\mathrm{X}$-ray LC, so there is only the normal decay; for GRB 080330 there are no observations after the plateau phase.

Group B. Some pairs of optical/X-ray slopes of the same GRB satisfy the relation $\alpha=0, \pm 1 / 4$. This group can be subdivided into two classes according to the LC shape: six GRBs have a similar shape for the X-ray and optical LCs, while 21 GRBs have different shapes.

GRBs with similar optical/X-ray LC shapes are GRB 051109, GRB 060526, GRB 071010A, GRB 060124, GRB 070208, and GRB 090618, where the optical and X-ray LC coincide during the X-ray plateau. In particular: the 060526 optical LC traces the X-ray LC at late time, even if the X-ray LC has few data points; the 090618 optical LC shows the presence of a supernova (SN) at late time (Dado \& Dar 2010; Cano et al. 2011b), so at late time optical and X-ray LCs do not coincide.

We divided GRBs with different optical-X-ray LC shapes into four subgroups: optical LC more complex than X-ray LC (five GRBs), X-ray LC more complex than optical LC (five GRBs), optical bumps (two GRBs), peculiar cases (nine GRBs). In the first subgroup are GRB 050319B (coincidence during the X-ray plateau phase), GRB 061121, and GRB 050408 (coincidence during the X-ray normal decay phase); the GRB 080413B optical LC resembles the canonical $\mathrm{X}$-ray shape and the first and last phases decay as the X-ray LC and the central part shows a plateau. The GRB 071031 normal decay has the same slope for the optical and X-ray LCs; we cannot conclude about the emission mechanism during this phase because the SEDs are too uncertain. There are two bumps superimposed on this segment. In the second subgroup are GRB 050401 and GRB 060502A (correspondence during the X-ray plateau phase), GRB 060908, GRB 060614, and GRB 091018 (correspondence during the X-ray normal decay phase). In the third group (GRB 060904B, GRB 080928) are the GRBs that show optical bumps that coincide with the X-ray steep and plateau phase; the optical and X-ray LC coincide during the normal decay phase (after the optical bumps). In the fourth subgroup are GRB $050525 \mathrm{~A}^{27}$, GRB 060512, GRB 080710, and GRB 090313, whose LCs coincide during the plateau phase; GRB 090926A, GRB 071003, GRB 081008, and GRB 081203A, whose LCs agree during the normal decay. The slopes of GRB 060729 optical LC are similar to the slopes of the X-ray LC during the plateau and the normal decay, but there is an optical re-brightening coincident with the end of the X-ray plateau.

Group C. No pairs of optical/X-ray slopes of the same GRB satisfy the relation $\alpha=0, \pm 1 / 4$. GRB 050730, GRB 060210, GRB 060605, GRB 060607A, GRB 070802, and GRB 080810 have type IIa X-ray LC and Ia optical LC (four with X-ray flares); 070529 has type IIa X-ray LC and Ib optical LC. Nine GRBs have a simple power-law X-ray LC (six truncated ${ }^{28}$ ): GRB 051111, GRB 061126, GRB 070125, GRB 070411, GRB 090102, GRB 090426, GRB 091127, GRB 070318, and GRB 060206. Eight GRBs have a Type Ia X-ray LC (four complete28): GRB 050908, GRB 060927, GRB 080721, GRB 081029, GRB 090424, GRB 050820A, GRB 050922C, and GRB 071112C. Only two GRBs have Type Ib X-ray LC (GRB 060418, GRB 071025) and one GRB has Type $I I b$ X-ray LC (GRB 070419B). GRB 060906 is observed in the optical band only during the X-ray plateau phase and it is not a regular shape, but there are optical bumps.

${ }^{27}$ In this case there is coincidence because the X-ray LC plateau slope has a relatively large error: $\alpha_{\mathrm{X}, \mathrm{PL}}=0.600 \pm 0.648$ and $\alpha_{\mathrm{op}}=1.150 \pm$ 0.036 .

28 As defined in M 13, complete X-ray LCs correspond to GRBs repointed by XRT at $t_{\text {rep }}<300 \mathrm{~s}$ for which we were able to follow the fading of the XRT flux down to a factor $\sim 5-10$ from the background limit (or, equivalently, $t_{\text {end }} \geq 4 \times 10^{5} \mathrm{~s}$ ). The LCs do not follow this criterion are classified as "truncated". 
E. Zaninoni et al.: The gamma-ray burst optical light-curve zoo: comparison with the X-ray observations

\section{References}

Akerlof, C. W., Kehoe, R. L., McKay, T. A., et al. 2003, PASP, 115, 132

Amati, L., Guidorzi, C., Frontera, F., et al. 2008, MNRAS, 391, 577

Andreev, M., Sergeev, A., Babina, J., et al. 2008, GRB Coordinates Network, 7655,1

Andreev, M., Sergeev, A., \& Pozanenko, A. 2010a, GRB Coordinates Network, 11166,1

Andreev, M., Sergeev, A., \& Pozanenko, A. 2010b, GRB Coordinates Network, 11168,1

Andreev, M., Sergeev, A., \& Pozanenko, A. 2010c, GRB Coordinates Network, 11191,1

Andreev, M., Sergeev, A., \& Pozanenko, A. 2010d, GRB Coordinates Network, 11201,1

Andreev, M., Sergeev, A., Pozanenko, A., et al. 2010e, GRB Coordinates Network, 11200, 1

Antonelli, L. A., D’Avanzo, P., Perna, R., et al. 2009, A\&A, 507, L45

Asfandyarov, I., Pozanenko, A., \& Ibrahimov, M. 2006, GRB Coordinates Network, 5434, 1

Barniol Duran, R., \& Kumar, P. 2009, MNRAS, 395, 955

Barthelmy, S. D., Barbier, L. M., Cummings, J. R., et al. 2005, Space Sci. Rev., 120,143

Behar, E., Dado, S., Dar, A., \& Laor, A. 2011, ApJ, 734, 26

Bernardini, M. G., Margutti, R., Chincarini, G., Guidorzi, C., \& Mao, J. 2011, A\&A, 526, A27

Bernardini, M. G., Margutti, R., Mao, J., Zaninoni, E., \& Chincarini, G. 2012a, A\&A, 539, A3

Bernardini, M. G., Margutti, R., Zaninoni, E., \& Chincarini, G. 2012b, MNRAS, 425,1199

Bikmaev, I., Khamitov, I., Melnikov, S., et al. 2010a, GRB Coordinates Network, 10635, 1

Bikmaev, I., Khamitov, I., Melnikov, S., et al. 2010b, GRB Coordinates Network, 10643, 1

Bikmaev, I., Khamitov, I., Melnikov, S., et al. 2010c, GRB Coordinates Network, 10700, 1

Bloom, J. S., Perley, D. A., Li, W., et al. 2009, ApJ, 691, 723

Boër, M., Atteia, J. L., Damerdji, Y., et al. 2006, ApJ, 638, L71

Boettcher, M., \& Joshi, M. 2005, GRB Coordinates Network, 4392, 1

Bohlin, R. C., Savage, B. D., \& Drake, J. F. 1978, ApJ, 224, 132

Boyd, P. T., \& Marshall, F. E. 2006, GRB Coordinates Network, 5357, 1

Breeveld, A. A. 2007, GRB Coordinates Network, 7028, 1

Breeveld, A. A., \& Grupe, D. 2008, GRB Coordinates Network, 8696, 1

Breeveld, A., Landsman, W., Kuin, P., \& Grupe, D. 2008, GRB Coordinates Network, 8712, 1

Brown, P. J., \& Baumgartner, W. H. 2008, GRB Coordinates Network, 8340, 1

Burrows, D. N., Hill, J. E., Nousek, J. A., et al. 2005, Space Sci. Rev., 120, 165

Butler, N. R., \& Kocevski, D. 2007, ApJ, 668, 400

Butler, N. R., Li, W., Perley, D., et al. 2006, ApJ, 652, 1390

Campana, S., Thöne, C. C., de Ugarte Postigo, A., et al. 2010, MNRAS, 402, 2429

Campana, S., Salvaterra, R., Melandri, A., et al. 2012, MNRAS, 421, 1697

Cano, Z., Guidorzi, C., Mundell, C., et al. 2009a, GRB Coordinates Network, 9779, 1

Cano, Z., Guidorzi, C., Mundell, C. G., et al. 2009b, GRB Coordinates Network, 10066, 1

Cano, Z., Bersier, D., Guidorzi, C., et al. 2011a, ApJ, 740, 41

Cano, Z., Bersier, D., Guidorzi, C., et al. 2011b, MNRAS, 413, 669

Cenko, S. B. 2009, GRB Coordinates Network, 9769, 1

Cenko, S. B., Kasliwal, M., Harrison, F. A., et al. 2006, ApJ, 652, 490

Cenko, S. B., Kelemen, J., Harrison, F. A., et al. 2009, ApJ, 693, 1484

Cenko, S. B., Frail, D. A., Harrison, F. A., et al. 2011, ApJ, 732, 29

Chandra, P., \& Frail, D. A. 2012, ApJ, 746, 156

Chandra, P., Cenko, S. B., Frail, D. A., et al. 2008, ApJ, 683, 924

Chevalier, R. A., \& Li, Z.-Y. 1999, ApJ, 520, L29

Chincarini, G., Zerbi, F., Antonelli, A., et al. 2003, The Messenger, 113, 40

Chincarini, G., Mao, J., Margutti, R., et al. 2010a, MNRAS, 406, 2113

Chincarini, G., Zannoni, M., Covino, S., et al. 2010b [arXiv: 1005. 1569]

Christie, G. W., Dong, S., de Ugarte Postigo, A., \& Natusch, T. 2009, GRB Coordinates Network, 10137, 1

Clemens, C., Kruehler, T., Greiner, J., et al. 2008a, GRB Coordinates Network, 7834, 1

Clemens, C., Kupcu Yoldas, A., Greiner, J., et al. 2008b, GRB Coordinates Network, 7851, 1

Cobb, B. E. 2006, GRB Coordinates Network, 5323, 1

Cobb, B. E. 2008a, GRB Coordinates Network, 7609, 1

Cobb, B. E. 2008b, GRB Coordinates Network, 8339, 1

Cobb, B. E. 2008c, GRB Coordinates Network, 8547, 1
Cobb, B. E. 2009a, GRB Coordinates Network, 9313, 1

Cobb, B. E. 2009b, GRB Coordinates Network, 10111, 1

Cobb, B. E., Bailyn, C. D., van Dokkum, P. G., \& Natarajan, P. 2006, ApJ, 651, L85

Cobb, B. E., Bloom, J. S., Perley, D. A., et al. 2010, ApJ, 718, L150

Corsi, A., \& Mészáros, P. 2009, ApJ, 702, 1171

Covino, S., D’ Avanzo, P., Klotz, A., et al. 2008, MNRAS, 388, 347

Covino, S., Campana, S., Conciatore, M. L., et al. 2010, A\&A, 521, A53

Curran, P. A., van der Horst, A. J., Beardmore, A. P., et al. 2007a, A\&A, 467, 1049

Curran, P. A., van der Horst, A. J., Wijers, R. A. M. J., et al. 2007b, MNRAS, 381, L65

Dado, S., \& Dar, A. 2010, ApJ, 708, L112

D'Agostini, G. 2005, e-print [arXiv: physics/0511182]

Dai, Z. G., \& Lu, T. 1998, A\&A, 333, L87

Dai, X., Halpern, J. P., Morgan, N. D., et al. 2007, ApJ, 658, 509

Dale, D., Barlow, R., Paul, C., et al. 2005, GRB Coordinates Network, 3957, 1

Dall'Osso, S., Stratta, G., Guetta, D., et al. 2011, A\&A, 526, A121

D’Avanzo, P., Malesani, D., Covino, S., et al. 2009, A\&A, 498, 711

D’Avanzo, P., Perri, M., Fugazza, D., et al. 2010, A\&A, 522, A20

De Pasquale, M., \& Parsons, A. 2008, GRB Coordinates Network, 8603, 1

De Pasquale, M., Beardmore, A. P., Barthelmy, S. D., et al. 2006, MNRAS, 365, 1031

de Ugarte Postigo, A., Fatkhullin, T. A., Jóhannesson, G., et al. 2007, A\&A, 462, L57

Della Valle, M., Chincarini, G., Panagia, N., et al. 2006, Nature, 444, 1050

Deng, J., Zheng, W., Zhai, M., et al. 2009, ApJ, submitted [arXiv: 0912 . 5435]

Dintinjana, B., Maticic, S., Mikuz, H., \& Skvarc, J. 2007, GRB Coordinates Network, 7078, 1

Draine, B. T. 2003, ARA\&A, 41, 241

Durig, D. T., McLarty, N. P., \& Manning, J. R. 2005, GRB Coordinates Network, 3950, 1

Elenin, L., Molotov, I., \& Pozanenko, A. 2010a, GRB Coordinates Network, 11184, 1

Elenin, L., Molotov, I., \& Pozanenko, A. 2010b, GRB Coordinates Network, 11234, 1

Elíasdóttir, Á., Fynbo, J. P. U., Hjorth, J., et al. 2009, ApJ, 697, 1725

Ferrero, P., Kann, D. A., Zeh, A., et al. 2006, A\&A, 457, 857

Ferrero, P., Klose, S., Kann, D. A., et al. 2009, A\&A, 497, 729

Filgas, R., Updike, A., \& Greiner, J. 2009, GRB Coordinates Network, 10098, 1

Filgas, R., Klose, S., \& Greiner, J. 2010, GRB Coordinates Network, 10617, 1

Filgas, R., Greiner, J., Schady, P., et al. 2011a, A\&A, 535, A57

Filgas, R., Krühler, T., Greiner, J., et al. 2011b, A\&A, 526, A113

Foley, R. J., Perley, D. A., Pooley, D., et al. 2006, ApJ, 645, 450

Freedman, D. L., \& Waxman, E. 2001, ApJ, 547, 922

Fukugita, M., Shimasaku, K., \& Ichikawa, T. 1995, PASP, 107, 945

Fynbo, J. P. U., Watson, D., Thöne, C. C., et al. 2006, Nature, 444, 1047

Fynbo, J. P. U., Jakobsson, P., Prochaska, J. X., et al. 2009, ApJS, 185, 526

Gehrels, N., Chincarini, G., Giommi, P., et al. 2004, ApJ, 611, 1005

Gendre, B., Klotz, A., Palazzi, E., et al. 2010, MNRAS, 405, 2372

George, K., Banerjee, D. P. K., Chandrasekhar, T., \& Ashok, N. M. 2006, ApJ, 640, L13

Ghirlanda, G., Nava, L., Ghisellini, G., et al. 2012, MNRAS, 420, 483

Goad, M. R., Tagliaferri, G., Page, K. L., et al. 2006, A\&A, 449, 89

Gomboc, A., Guidorzi, C., Mundell, C. G., et al. 2006, Nuovo Cimento B Serie, 121,1303

Gomboc, A., Guidorzi, C., Melandri, A., et al. 2008a, GRB Coordinates Network, 7625, 1

Gomboc, A., Guidorzi, C., Melandri, A., et al. 2008b, GRB Coordinates Network, 7626, 1

Gomboc, A., Kobayashi, S., Guidorzi, C., et al. 2008c, ApJ, 687, 443

Gorbovskoy, E. S., Lipunova, G. V., Lipunov, V. M., et al. 2012, MNRAS, 421, 1874

Gou, L.-J., Fox, D. B., \& Mészáros, P. 2007, ApJ, 668, 1083

Graham, J. F., Fruchter, A. S., Levan, A. J., et al. 2009, ApJ, 698, 1620

Granot, J., Piran, T., \& Sari, R. 2000, ApJ, 534, L163

Greco, G., Terra, F., Bartolini, C., et al. 2007, GRB Coordinates Network, 7089, 1

Greiner, J., Bornemann, W., Clemens, C., et al. 2008, PASP, 120, 405

Greiner, J., Krühler, T., Fynbo, J. P. U., et al. 2009a, ApJ, 693, 1610

Greiner, J., Krühler, T., McBreen, S., et al. 2009b, ApJ, 693, 1912

Greiner, J., Krühler, T., Klose, S., et al. 2011, A\&A, 526, A30

Grupe, D., Brown, P. J., Cummings, J., et al. 2006, ApJ, 645, 464

Grupe, D., Gronwall, C., Wang, X.-Y., et al. 2007, ApJ, 662, 443

Guidorzi, C., \& Steele, I. 2008, GRB Coordinates Network, 8064, 1

Guidorzi, C., Gomboc, A., Kobayashi, S., et al. 2007, A\&A, 463, 539

Guidorzi, C., Clemens, C., Kobayashi, S., et al. 2009, A\&A, 499, 439

Guidorzi, C., Kobayashi, S., Perley, D. A., et al. 2011, MNRAS, 417, 2124 
Haislip, J. B., Nysewander, M. C., Reichart, D. E., et al. 2006, Nature, 440, 181

Haislip, J., Reichart, D., Ivarsen, K., et al. 2009a, GRB Coordinates Network, 9999, 1

Haislip, J., Reichart, D., Ivarsen, K., et al. 2009b, GRB Coordinates Network, 9916, 1

Halpern, J., \& Armstrong, E. 2006a, GRB Coordinates Network, 5853, 1

Halpern, J., \& Armstrong, E. 2006b, GRB Coordinates Network, 5851, 1

Halpern, J. P., Mirabal, N., \& Armstrong, E. 2006a, GRB Coordinates Network, 5847, 1

Halpern, J. P., Mirabal, N., \& Armstrong, E. 2006b, GRB Coordinates Network, 5840, 1

Henden, A., Gross, J., Denny, B., Terrell, D., \& Cooney, W. 2009, GRB Coordinates Network, 10073, 1

Hentunen, V.-P., Oksanen, A., \& Kehusmaa, P. 2008, GRB Coordinates Network, 7484, 1

Hentunen, V.-P., Nissinen, M., \& Salmi, T. 2010, GRB Coordinates Network, 11173,1

Holland, S. T., \& Pagani, C. 2008, GRB Coordinates Network, 7497, 1

Holland, S. T., Boyd, P. T., Gorosabel, J., et al. 2007, AJ, 133, 122

Holland, S. T., De Pasquale, M., Mao, J., et al. 2012, ApJ, 745, 41

Hoversten, E. A., \& Cummings, J. R. 2008, GRB Coordinates Network, 7398,

Huang, K. Y., Urata, Y., Kuo, P. H., et al. 2007, ApJ, 654, L25

Hurkett, C. P., Osborne, J. P., Page, K. L., et al. 2006, MNRAS, 368, 1101

Im, M., Jeon, Y., Park, W., et al. 2009, GRB Coordinates Network, 9275, 1

Ishimura, T., Shimokawabe, T., Mori, Y., et al. 2007, GRB Coordinates Network, 7087, 1

Ishimura, T., Kawai, N., Kotani, T., et al. 2008, in AIP Conf. Ser., 1000, eds. M. Galassi, D. Palmer, \& E. Fenimore, 261

Isogai, M., \& Kawai, N. 2008, GRB Coordinates Network, 8629, 1

Jakobsson, P., Hjorth, J., Ramirez-Ruiz, E., et al. 2004, New Astron., 9, 435

Jakobsson, P., Fynbo, J. P. U., Hjorth, J., et al. 2006a, GRB Coordinates Network, 5337, 1

Jakobsson, P., Fynbo, J. P. U., Tanvir, N., et al. 2006b, GRB Coordinates Network, 5355, 1

Jaunsen, A. O., Rol, E., Watson, D. J., et al. 2008, ApJ, 681, 453

Kalberla, P. M. W., Burton, W. B., Hartmann, D., et al. 2005, A\&A, 440, 775

Kamble, A., Resmi, L., \& Misra, K. 2007, ApJ, 664, L5

Kamble, A., Misra, K., Bhattacharya, D., \& Sagar, R. 2009, MNRAS, 394, 214

Kann, D. A., Klose, S., \& Zeh, A. 2006, ApJ, 641, 993

Kann, D. A., Hoegner, C., \& Filgas, R. 2007a, GRB Coordinates Network, 6884, 1

Kann, D. A., Hoegner, C., \& Filgas, R. 2007b, GRB Coordinates Network, 6918, 1

Kann, D. A., Laux, U., \& Filgas, R. 2007c, GRB Coordinates Network, 6923, 1 Kann, D. A., Laux, U., Filgas, R., et al. 2007d, GRB Coordinates Network, 6935, 1

Kann, D. A., Laux, U., Klose, S., et al. 2007e, GRB Coordinates Network, 6295, 1

Kann, D. A., Laux, U., \& Ertel, S. 2008, GRB Coordinates Network, 7845, 1

Kann, D. A., Klose, S., Laux, U., \& Stecklum, B. 2010a, GRB Coordinates Network, 11187, 1

Kann, D. A., Klose, S., Zhang, B., et al. 2010b, ApJ, 720, 1513

Kann, D. A., Laux, U., \& Stecklum, B. 2010c, GRB Coordinates Network, 11236, 1

Kann, D. A., Nicuesa Guelbenzu, A., Ludwig, F., \& Stecklum, B. 2010d, GRB Coordinates Network, 11247, 1

Kann, D. A., Klose, S., Zhang, B., et al. 2011, ApJ, 734, 96

Kelemen, J., Sarneczky, K., Kuli, Z., \& Ujhelyi, B. 2010, GRB Coordinates Network, 11252, 1

Khamitov, I. M., Burenin, R. A., Bikmaev, I. F., et al. 2007, Astron. Lett., 33, 797

Khamitov, I., Bikmaev, I., Gumerov, R., et al. 2008, GRB Coordinates Network, 7661,1

Kinugasa, K. 2008, GRB Coordinates Network, 7413, 1

Klein, C. R., Morgan, A. N., Perley, D. A., \& Bloom, J. S. 2010, GRB Coordinates Network, 10627, 1

Klotz, A., Boer, M., \& Atteia, J. L. 2005a, GRB Coordinates Network, 3720, 1

Klotz, A., Boër, M., Atteia, J. L., et al. 2005b, A\&A, 439, L35

Klotz, A., Boer, M., \& Atteia, J. L. 2008a, GRB Coordinates Network, 7595, 1

Klotz, A., Vachier, F., \& Boër, M. 2008b, Astron. Nachr., 329, 275

Klotz, A., Boër, M., Atteia, J. L., \& Gendre, B. 2009a, AJ, 137, 4100

Klotz, A., Gendre, B., Boer, M., \& Atteia, J. L. 2009b, GRB Coordinates Network, 8998, 1

Klotz, A., Gendre, B., Boer, M., \& Atteia, J. L. 2009c, GRB Coordinates Network, 10200, 1

Kocevski, D., Modjaz, M., Bloom, J. S., et al. 2007, ApJ, 663, 1180

Krimm, H. A., Granot, J., Marshall, F. E., et al. 2007, ApJ, 665, 554
Kruehler, T., Schrey, F., Greiner, J., et al. 2008, GRB Coordinates Network, 8075,1

Krühler, T., Küpcü Yoldaş, A., Greiner, J., et al. 2008, ApJ, 685, 376

Krühler, T., Greiner, J., Afonso, P., et al. 2009a, A\&A, 508, 593

Krühler, T., Greiner, J., McBreen, S., et al. 2009b, ApJ, 697, 758

Kuin, N. P. M., \& Mangano, V. 2008a, GRB Coordinates Network, 7804, 1

Kuin, N. P. M., \& Mangano, V. 2008b, GRB Coordinates Network, 7808, 1

Kuin, N. P. M., \& Racusin, J. L. 2008, GRB Coordinates Network, 8069, 1

Kuin, N. P. M., Sbarufatti, B., Marshall, F., \& Schady, P. 2008, GRB Coordinates Network, 7844, 1

Kumar, P., \& Panaitescu, A. 2000, ApJ, 541, L51

Kumar, P., Narayan, R., \& Johnson, J. L. 2008, MNRAS, 388, 1729

Kuroda, D., Yoshida, M., Yanagisawa, K., et al. 2008, GRB Coordinates Network, 8724, 1

Kuroda, D., Yanagisawa, K., Shimizu, Y., et al. 2010a, GRB Coordinates Network, 11186, 1

Kuroda, D., Yanagisawa, K., Shimizu, Y., et al. 2010b, GRB Coordinates Network, 11172, 1

Kuroda, D., Yanagisawa, K., Shimizu, Y., et al. 2010c, GRB Coordinates Network, 11189, 1

Kuroda, D., Yanagisawa, K., Shimizu, Y., et al. 2010d, GRB Coordinates Network, 11241, 1

Lacluyze, A., Reichart, D., Haislip, J., et al. 2009, GRB Coordinates Network, 10107, 1

Landsman, W. B., \& Guidorzi, C. 2008, GRB Coordinates Network, 7660, 1

Landsman, W. B., \& Sbarufatti, B. 2008, GRB Coordinates Network, 7965, 1

Lazzati, D., Rossi, E., Covino, S., Ghisellini, G., \& Malesani, D. 2002, A\&A, 396, L5

Li, W. 2005, GRB Coordinates Network, 4240, 1

Li, W., \& Filippenko, A. V. 2008, GRB Coordinates Network, 7475, 1

Li, W., Perley, D. A., \& Filippenko, A. V. 2009, GRB Coordinates Network, 9517, 1

Li, L., Liang, E.-W., Tang, Q.-W., et al. 2012, ApJ, 758, 27

Liang, E., \& Zhang, B. 2006, MNRAS, 369, L37

Liang, E.-W., Li, L., Gao, H., et al. 2012, ApJ, submitted [arXiv: 1210. 5142]

Lipunov, V. M., Kornilov, V. G., Krylov, A. V., et al. 2007, Astron. Rep., 51, 1004

Loew, S., Kruehler, T., \& Greiner, J. 2008, GRB Coordinates Network, 8540, 1

Malesani, D., Covino, S., D’Avanzo, P., et al. 2007, A\&A, 473, 77

Mangano, V., Holland, S. T., Malesani, D., et al. 2007, A\&A, 470, 105

Mao, J., Cha, G., \& Bai, J. 2009a, GRB Coordinates Network, 9305, 1

Mao, J., Li, S., \& Bai, J. 2009b, GRB Coordinates Network, 10092, 1

Margutti, R., Genet, F., Granot, J., et al. 2010a, MNRAS, 402, 46

Margutti, R., Guidorzi, C., Chincarini, G., et al. 2010b, MNRAS, 406, 2149

Margutti, R., Zaninoni, E., Bernardini, M. G., et al. 2013, MNRAS, 428, 729

Markwardt, C. B. 2009, in Astronomical Data Analysis Software and Systems XVIII, eds. D. A. Bohlender, D. Durand, \& P. Dowler, ASP Conf. Ser., 411, 251

Marshall, F. E., \& Grupe, D. 2009, GRB Coordinates Network, 10108, 1

Marshall, F. E., Antonelli, L. A., Burrows, D. N., et al. 2011, ApJ, 727, 132

Martin, N., Maurice, E., \& Lequeux, J. 1989, A\&A, 215, 219

Mason, K. O., Blustin, A. J., Boyd, P., et al. 2006, ApJ, 639, 311

McBreen, S., Krühler, T., Rau, A., et al. 2010, A\&A, 516, A71

Melady, G., French, J., Kubanek, P., \& Jelinek, M. 2007, GRB Coordinates Network, 6528, 1

Melandri, A., Tanvir, N., \& Guidorzi, C. 2006, GRB Coordinates Network, 5322, 1

Melandri, A., Guidorzi, C., \& Carter, D. 2008a, GRB Coordinates Network, 8699, 1

Melandri, A., Mundell, C. G., Kobayashi, S., et al. 2008b, ApJ, 686, 1209

Melandri, A., Guidorzi, C., Kobayashi, S., et al. 2009, MNRAS, 395, 1941

Melandri, A., Kobayashi, S., Mundell, C. G., et al. 2010, ApJ, 723, 1331

Mikuz, H., Skvarc, J., \& Dintinjana, B. 2007, GRB Coordinates Network, 6288,1

Minezaki, T., Price, P. A., Yoshii, Y., \& Cowie, L. L. 2007, GRB Coordinates Network, 7135, 1

Mirabal, N., Halpern, J. P., An, D., Thorstensen, J. R., \& Terndrup, D. M. 2006, ApJ, 643, L99

Misra, K., Bhattacharya, D., Sahu, D. K., et al. 2007, A\&A, 464, 903

Molinari, E., Vergani, S. D., Malesani, D., et al. 2007, A\&A, 469, L13

Monfardini, A., Kobayashi, S., Guidorzi, C., et al. 2006, ApJ, 648, 1125

Moody, J. W., Laney, D., Pearson, R., \& Pace, C. 2010, GRB Coordinates Network, 10665, 1

Morgan, A. N., Bloom, J. S., Perley, D. A., \& Starr, D. 2009a, GRB Coordinates Network, 8995, 1

Morgan, A. N., Klein, C. R., \& Bloom, J. S. 2009b, GRB Coordinates Network, 9563,1 
Morgan, A. N., Perley, D. A., Klein, C. R., \& Bloom, J. S. 2010, GRB Coordinates Network, 10648, 1

Mori, Y. A., Nakajima, H., Shimokawabe, T., et al. 2008, GRB Coordinates Network, 8619, 1

Morrison, R., \& McCammon, D. 1983, ApJ, 270, 119

Mundell, C. G., Melandri, A., Guidorzi, C., et al. 2007, ApJ, 660, 489

Nardini, M., Ghisellini, G., Ghirlanda, G., et al. 2006, A\&A, 451, 821

Nardini, M., Ghisellini, G., \& Ghirlanda, G. 2008, MNRAS, 383, 1049

Nardini, M., Greiner, J., Krühler, T., et al. 2011, A\&A, 531, A39

Nicuesa Guelbenzu, A., Klose, S., Rossi, A., et al. 2011, A\&A, 531, L6

Nissinen, M. 2009, GRB Coordinates Network, 8993, 1

Nissinen, M., \& Hentunen, V.-P. 2009, GRB Coordinates Network, 9246, 1

Nousek, J. A., Kouveliotou, C., Grupe, D., et al. 2006, ApJ, 642, 389

Novak, R. 2008, GRB Coordinates Network, 7504, 1

Nysewander, M., Fruchter, A. S., \& Pe'er, A. 2009a, ApJ, 701, 824

Nysewander, M., Reichart, D. E., Crain, J. A., et al. 2009b, ApJ, 693, 1417

Oates, S. R. 2008, GRB Coordinates Network, 8544, 1

Oates, S. R., \& Marshall, F. E. 2008, GRB Coordinates Network, 7607, 1

Oates, S. R., \& Marshall, F. E. 2009, GRB Coordinates Network, 10078, 1

Oates, S. R., \& Stamatikos, M. 2008, GRB Coordinates Network, 7611, 1

Oates, S. R., \& Stratta, G. 2007, GRB Coordinates Network, 7080, 1

Oates, S. R., Page, M. J., Schady, P., et al. 2009, MNRAS, 395, 490

Oates, S. R., Page, M. J., Schady, P., et al. 2011, MNRAS, 412, 561

Oates, S. R., Page, M. J., De Pasquale, M., et al. 2012, MNRAS, 426, L86

Oksanen, A., \& Hentunen, V.-P. 2008, GRB Coordinates Network, 7657, 1

Oksanen, A., Templeton, M., Henden, A. A., \& Kann, D. A. 2008, Journal of the

American Association of Variable Star Observers (JAAVSO), 36, 53

Olivares, F., Kupcu Yoldas, A., Greiner, J., \& Yoldas, A. 2009, GRB Coordinates Network, 9245, 1

Page, K. L., Willingale, R., Bissaldi, E., et al. 2009, MNRAS, 400, 134

Panaitescu, A., \& Vestrand, W. T. 2008, MNRAS, 387, 497

Panaitescu, A., \& Vestrand, W. T. 2011, MNRAS, 414, 3537

Pandey, S. B., Castro-Tirado, A. J., McBreen, S., et al. 2006, A\&A, 460, 415

Pandey, S. B., Castro-Tirado, A. J., Jelínek, M., et al. 2009, A\&A, 504, 45 Pandey, S. B., Swenson, C. A., Perley, D. A., et al. 2010, ApJ, 714, 799

Pei, Y. C. 1992, ApJ, 395, 130

Perley, D. A. 2009, GRB Coordinates Network, 8997, 1

Perley, D. A., \& Bloom, J. S. 2008, GRB Coordinates Network, 7233, 1

Perley, D. A., Bloom, J. S., \& Li, W. 2008a, GRB Coordinates Network, 7406, 1

Perley, D. A., Li, W., Chornock, R., et al. 2008b, ApJ, 688, 470

Perley, D. A., Cenko, S. B., Bloom, J. S., et al. 2009a, AJ, 138, 1690

Perley, D. A., Cenko, S. B., Bloom, J. S., Li, W., \& Cobb, B. E. 2009b, GRB Coordinates Network, 9001, 1

Perley, D. A., Bloom, J. S., Klein, C. R., et al. 2010, MNRAS, 406, 2473

Perley, D. A., Morgan, A. N., Updike, A., et al. 2011, AJ, 141, 36

Perri, M., Guetta, D., Antonelli, L. A., et al. 2007, A\&A, 471, 83

Piran, T., Kumar, P., Panaitescu, A., \& Piro, L. 2001, ApJ, 560, L167

Poole, T. S., Breeveld, A. A., Page, M. J., et al. 2008, MNRAS, 383, 627

Price, P. A., Cowie, L. L., Minezaki, T., et al. 2006, ApJ, 645, 851

Pritchard, T. A., \& Immler, S. 2010, GRB Coordinates Network, 11176, 1

Quimby, R. M., Rykoff, E. S., Yost, S. A., et al. 2006, ApJ, 640, 402

Racusin, J. L., Karpov, S. V., Sokolowski, M., et al. 2008, Nature, 455, 183

Rau, A., Savaglio, S., Krühler, T., et al. 2010, ApJ, 720, 862

Romano, P., Campana, S., Chincarini, G., et al. 2006, A\&A, 456, 917

Roming, P. W. A., Koch, T. S., Oates, S. R., et al. 2009, ApJ, 690, 163

Rossi, A., Schulze, S., Klose, S., et al. 2011, A\&A, 529, A142

Rowlinson, A., Wiersema, K., Levan, A. J., et al. 2010, MNRAS, 408, 383

Ruiz-Velasco, A. E., Swan, H., Troja, E., et al. 2007, ApJ, 669, 1

Rumyantsev, V., \& Pozanenko, A. 2008a, GRB Coordinates Network, 7857,

1

Rumyantsev, V., \& Pozanenko, A. 2008b, GRB Coordinates Network, 7833, 1

Rumyantsev, V., Pavlenko, E., \& Pozanenko, A. 2006, GRB Coordinates Network, 5336, 1

Rumyantsev, V., Antonyuk, K., Andreev, M., \& Pozanenko, A. 2008, GRB Coordinates Network, 8645, 1

Rumyantsev, V., Biryukov, V., \& Pozanenko, A. 2009, GRB Coordinates Network, 10116, 1

Rumyantsev, V., Shakhovkoy, D., \& Pozanenko, A. 2010, GRB Coordinates Network, 10634, 1

Rykoff, E. S., Yost, S. A., Krimm, H. A., et al. 2005, ApJ, 631, L121

Rykoff, E. S., Rujopakarn, W., Quimby, R., et al. 2007, GRB Coordinates Network, 6269, 1

Rykoff, E. S., Aharonian, F., Akerlof, C. W., et al. 2009, ApJ, 702, 489

Sahu, D. K., Arora, S., Singh, N. S., \& Kartha, S. S. 2010a, GRB Coordinates Network, 11197, 1
Sahu, D. K., Bhatt, B. C., \& Arora, S. 2010b, GRB Coordinates Network, 11175, 1

Sari, R., Piran, T., \& Narayan, R. 1998, ApJ, 497, L17

Schady, P., \& Cannizzo, J. K. 2009, GRB Coordinates Network, 9234, 1

Schady, P., \& Stamatikos, M. 2009, GRB Coordinates Network, 9774, 1

Schady, P., Page, M. J., Oates, S. R., et al. 2010, MNRAS, 401, 2773

Schady, P., Dwelly, T., Page, M. J., et al. 2012, A\&A, 537, A15

Schlegel, D. J., Finkbeiner, D. P., \& Davis, M. 1998, ApJ, 500, 525

Schneider, D. P., Gunn, J. E., \& Hoessel, J. G. 1983, ApJ, 264, 337

Schulze, S., Klose, S., Björnsson, G., et al. 2011, A\&A, 526, A23

Siegel, M. H., \& Markwardt, C. B. 2010, GRB Coordinates Network, 11242,

Siegel, M. H., \& Marshall, F. 2010, GRB Coordinates Network, 10625, 1

Siegel, M. H., Marshall, F. E., Holland, S. T., et al. 2010, GRB Coordinates Network, 10645, 1

Smith, R. J., Melandri, A., Steele, I. A., et al. 2008, GRB Coordinates Network, 8333, 1

Smith, R. J., Gomboc, A., Guidorzi, C., et al. 2009, GRB Coordinates Network, 9770, 1

Soderberg, A. M., Berger, E., Kasliwal, M., et al. 2006, ApJ, 650, 261

Soderberg, A. M., Nakar, E., Cenko, S. B., et al. 2007, ApJ, 661, 982

Sollerman, J., Fynbo, J. P. U., Gorosabel, J., et al. 2007, A\&A, 466, 839

Sposetti, S. 2007, GRB Coordinates Network, 7090, 1

Sposetti, S., \& Immler, S. 2010, GRB Coordinates Network, 11213, 1

Stanek, K. Z., Dai, X., Prieto, J. L., et al. 2007, ApJ, 654, L21

Starling, R. L. C., Rol, E., van der Horst, A. J., et al. 2009, MNRAS, 400, 90

Stefanescu, A., Schrey, F., Duscha, S., et al. 2006, GRB Coordinates Network, 5623,1

Still, M., Roming, P. W. A., Mason, K. O., et al. 2005, ApJ, 635, 1187

Swan, H., Yuan, F., \& Rujopakarn, W. 2008, GRB Coordinates Network, 7470, 1

Tagliaferri, G., Antonelli, L. A., Chincarini, G., et al. 2005a, A\&A, 443, L1

Tagliaferri, G., Goad, M., Chincarini, G., et al. 2005b, Nature, 436, 985

Terra, F., Munz, F., Greco, G., et al. 2008, GRB Coordinates Network, 8368, 1

Thoene, C. C., Fynbo, J. P. U., de Ugarte Postigo, A., et al. 2008, GRB Coordinates Network, 7658, 1

Thöne, C. C., Kann, D. A., Jóhannesson, G., et al. 2010, A\&A, 523, A70

Tkachenko, A., Khamitov, I., Burenin, R., et al. 2010, GRB Coordinates Network, 11254, 1

Troja, E., Cusumano, G., O'Brien, P. T., et al. 2007, ApJ, 665, 599

Uehara, T., Uemura, M., Kawabata, K. S., et al. 2010, A\&A, 519, A56

Uehara, T., Uemura, M., Arai, A., et al. 2011, A\&A, 526, A92

Updike, A., Afonso, P., Clemens, C., \& Greiner, J. 2008a, GRB Coordinates Network, 8693, 1

Updike, A. C., Haislip, J. B., Nysewander, M. C., et al. 2008b, ApJ, 685, 361

Updike, A., Rau, A., Kruehler, T., Olivares, F., \& Greiner, J. 2009a, GRB Coordinates Network, 9773, 1

Updike, A. C., Hartmann, D. H., Milne, P. A., \& Williams, G. G. 2009b, GRB Coordinates Network, 10074, 1

Updike, A. C., Klose, S., Clemens, C., \& Greiner, J. 2009c, GRB Coordinates Network, 8983, 1

Urata, Y., Yamazaki, R., Sakamoto, T., et al. 2007, ApJ, 668, L95

Šimon, V., Polášek, C., Jelínek, M., Hudec, R., \& Trobl, J. A.. 2010, A\&A, 510, A49

Vaalsta, T. P., Coward, D. M., Zadko, J., et al. 2009, GRB Coordinates Network, 8976,1

Vestrand, W. T., Wren, J. A., Wozniak, P. R., et al. 2006, Nature, 442, 172

Volnova, A., Msu, S., Ibrahimov, M., Karimov, R., \& Pozanenko, A. 2010, GRB Coordinates Network, 10821, 1

Wang, J. H., Schwamb, M. E., Huang, K. Y., et al. 2008, ApJ, 679, L5

Watson, D., Fynbo, J. P. U., Ledoux, C., et al. 2006, ApJ, 652, 1011

Watson, D., Zafar, T., Andersen, A. C., et al. 2013, ApJ, 768, 23

Wegner, G., Garnavich, P., Prieto, J. L., \& Stanek, K. Z. 2008, GRB Coordinates Network, 7423, 1

Weingartner, J. C., \& Draine, B. T. 2001, ApJ, 548, 296

West, J. P., McLin, K., Brennan, T., et al. 2008, GRB Coordinates Network, 8617,1

Wiersema, K., Curran, P. A., Krühler, T., et al. 2012, MNRAS, 426, 2

Williams, G. G., \& Milne, P. A. 2008, GRB Coordinates Network, 7443, 1

Woźniak, P. R., Vestrand, W. T., Wren, J. A., et al. 2005, ApJ, 627, L13

Woźniak, P. R., Vestrand, W. T., Wren, J. A., et al. 2006, ApJ, 642, L99

Woźniak, P. R., Vestrand, W. T., Panaitescu, A. D., et al. 2009, ApJ, 691, 495

Wren, J., Vestrand, W. T., Wozniak, P. R., \& Davis, H. 2008a, GRB Coordinates Network, 7477, 1

Wren, J., Vestrand, W. T., Wozniak, P. R., Davis, H., \& Norman, B. 2008b, GRB Coordinates Network, 8337, 1 
A\&A 557, A12 (2013)

Wren, J., Vestrand, W. T., Wozniak, P. R., Davis, H., \& Norman, B. 2009, GRB Coordinates Network, 9778, 1

Xin, L.-P., Liang, E.-W., Wei, J.-Y., et al. 2011, MNRAS, 410, 27

Xu, D., Starling, R. L. C., Fynbo, J. P. U., et al. 2009, ApJ, 696, 971

Yoshida, M., Yanagisawa, K., Kuroda, D., et al. 2008a, GRB Coordinates Network, 7863, 1

Yoshida, M., Yanagisawa, K., Shimizu, Y., et al. 2008b, GRB Coordinates Network, 7410, 1

Yoshida, M., Sasada, M., Komatsu, T., \& Kawabata, K. S. 2010, GRB Coordinates Network, 11190, 1

Yost, S. A., Aharonian, F., Akerlof, C. W., et al. 2007a, ApJ, 669, 1107

Yost, S. A., Swan, H. F., Rykoff, E. S., et al. 2007b, ApJ, 657, 925
Yuan, F., Rykoff, E. S., Rujopakarn, W., \& Swan, H. 2007, GRB Coordinates Network, 7061, 1

Yuan, F., Quimby, R., Swan, H., \& Akerlof, C. 2008, GRB Coordinates Network, 7411,1

Yuan, F., Schady, P., Racusin, J. L., et al. 2010, ApJ, 711, 870

Zafar, T., Watson, D., Fynbo, J. P. U., et al. 2011, A\&A, 532, A143

Zerbi, R. M., Chincarini, G., Ghisellini, G., et al. 2001, Astron. Nachr., 322, 275

Zhang, B., \& Mészáros, P. 2001, ApJ, 552, L35

Zhang, B., Fan, Y. Z., Dyks, J., et al. 2006, ApJ, 642, 354

Ziaeepour, H., Holland, S. T., Boyd, P. T., et al. 2008, MNRAS, 385, 453

Pages 23 to 55 are available in the electronic edition of the journal at http://www. aanda. org 
E. Zaninoni et al.: The gamma-ray burst optical light-curve zoo: comparison with the X-ray observations

\section{Appendix C: Tables and figures}

We provide here:

- the examples of the online tables of CDS: the optical LC fit parameters (Tables C.1); the parameters of the optical/X-ray SEDs fitted with a single power-law (Table C.2) and with a broken power-law (Table C.3); the X-ray spectrum fit parameters (Table C.4); redshifts and luminosity distances of the GRBs in our sample (Table C.5); the optical data used for the SEDs (Table C.6);

- the result of the F-test over the optical/X-ray SEDs to choose the better fit function (Table C.8);

- the factors to convert magnitudes into flux densities (Table C.7);

- the sample of the 165 GRBs with known redshift from which we started the data selection, with references to papers and GCNs whith optical data (Table C.9);

- plots of the optical and X-ray LCs for the GRBs in our sample (Figs. C.1-C.9);

- plots of the optical/X-ray SEDs (Figs. C.10-C.18).

Table C.1. Optical LC fit parameters.

\begin{tabular}{|c|c|c|c|c|c|c|c|c|c|c|c|c|c|c|c|c|c|}
\hline & & GRF & & Fil Fit & ok & $T_{\min }$ & $T_{\max }$ & $t_{\mathrm{br}, 1}$ & $\sigma\left(t_{\mathrm{br}, 1}\right)$ & $t_{\mathrm{br}, 2}$ & $\sigma\left(t_{\mathrm{br}, 2}\right)$ & $t_{\mathrm{br}, 3}$ & $\sigma\left(t_{\mathrm{br}, 3}\right)$ & $\alpha 1$ & \multicolumn{2}{|c|}{$\sigma\left(\alpha_{1}\right)$} & \\
\hline & & $\overline{050}$ & 319 & 0 & 0 & 243.0 & 28491.0 & -9.0 & -9.0 & -9.0 & -9.0 & -9.0 & -9.0 & $7.040 \mathrm{e}-0$ & \multicolumn{2}{|c|}{$7.955 \mathrm{e}-02$} & \\
\hline & & 050 & 319 & 0 & 0 & 259.17 & 767337.7 & -9.0 & -9.0 & -9.0 & -9.0 & -9.0 & -9.0 & $6.618 \mathrm{e}-0$ & \multicolumn{2}{|c|}{$2.600 \mathrm{e}-02$} & \\
\hline & & 050 & 319 & BJ 0 & 0 & 5203.9 & 57196.8 & -9.0 & -9.0 & -9.0 & -9.0 & -9.0 & -9.0 & $4.112 \mathrm{e}-0$ & \multicolumn{2}{|c|}{$4.117 \mathrm{e}-02$} & \\
\hline & & 050 & 319 & VJ 0 & 0 & 6944.0 & 400550.4 & -9.0 & -9.0 & -9.0 & -9.0 & -9.0 & -9.0 & $6.905 \mathrm{e}-0$ & \multicolumn{2}{|c|}{$1.928 \mathrm{e}-02$} & \\
\hline & & 050 & 319 & RC 2 & 1 & 40.3 & $994118.4 \quad 15$ & 157243.4 & $+\quad 19520.9$ & 482.5 & 30.1 & -9.0 & -9.0 & $4.457 \mathrm{e}-0$ & \multicolumn{2}{|c|}{$1.429 \mathrm{e}-02$} & \\
\hline & $\alpha_{2}$ & & \multicolumn{2}{|c|}{$\sigma\left(\alpha_{2}\right)$} & \multicolumn{2}{|r|}{$\alpha_{3}$} & $\sigma\left(\alpha_{3}\right)$ & \multicolumn{2}{|c|}{$\alpha_{4}$} & $\sigma\left(\alpha_{4}\right)$ & \multicolumn{2}{|c|}{$\alpha_{5}$} & \multicolumn{2}{|c|}{$\sigma\left(\alpha_{5}\right)$} & $\overline{\alpha_{6}}$ & \multicolumn{2}{|c|}{$\sigma\left(\alpha_{6}\right)$} \\
\hline & \multicolumn{2}{|c|}{$-9.000 \mathrm{e}+00$} & \multicolumn{2}{|c|}{$-9.000 \mathrm{e}+00$} & \multicolumn{2}{|c|}{$-9.000 \mathrm{e}+00$} & $-9.000 \mathrm{e}+00$ & \multicolumn{2}{|c|}{$-9.000 \mathrm{e}+00$} & $.000 \mathrm{e}+0$ & \multicolumn{2}{|c|}{$0-9.000 \mathrm{e}+00$} & \multicolumn{2}{|c|}{$-9.000 e+00$} & $.000 \mathrm{e}+00$ & \multicolumn{2}{|c|}{$-9.000 \mathrm{e}+00$} \\
\hline & \multirow{2}{*}{\multicolumn{2}{|c|}{$\begin{array}{l}-9.000 e+00 \\
-9.000 e+00\end{array}$}} & \multirow{2}{*}{\multicolumn{2}{|c|}{$-9.000 \mathrm{e}+00$}} & \multicolumn{2}{|c|}{$-9.000 e+00$} & $-9.000 e+00$ & \multicolumn{2}{|c|}{$-9.000 e+00$} & $.000 \mathrm{e}+0$ & \multicolumn{2}{|c|}{$-9.000 e+00$} & \multicolumn{2}{|c|}{$-9.000 e+00-9$. } & $.000 \mathrm{e}+00$ & \multicolumn{2}{|c|}{$-9.000 \mathrm{e}+00$} \\
\hline & & & & & -9.00 & $00 \mathrm{e}+00$ & $-9.000 \mathrm{e}+00$ & \multicolumn{2}{|c|}{$-9.000 \mathrm{e}+00-9$} & $.000 \mathrm{e}+0$ & \multicolumn{2}{|c|}{$-9.000 \mathrm{e}+00$} & $-9.000 \mathrm{e}$ & $+00 \quad-9$. & $.000 \mathrm{e}+00$ & -9.00 & \\
\hline & -9.000 & ee+00 & -9.0 & $000 \mathrm{e}+00$ & -9.00 & $00 \mathrm{e}+00$ & $-9.000 e+00$ & -9.000 & $0 \mathrm{e}+00-9$. & $.000 \mathrm{e}+0$ & $\begin{array}{ll}00 & -9.000\end{array}$ & $0 \mathrm{e}+00$ & $-9.000 \mathrm{e}$ & $+00 \quad-9$ & $.000 \mathrm{e}+00$ & -9.0 & \\
\hline & $1.559 \mathrm{e}$ & & & $39 \mathrm{e}-01$ & 2.52 & $20 \mathrm{e}+00$ & $2.744 \mathrm{e}-01$ & 1.240 & e- 01 & $844 \mathrm{e}-02$ & $2 \quad-9.000$ & $0 \mathrm{e}+00$ & $-9.000 \mathrm{e}$ & $+00 \quad-9$. & $.000 \mathrm{e}+00$ & -9 . & 00 \\
\hline$S_{1}$ & $s_{2}$ & $s_{3}$ & & $N_{1}$ & & $\sigma\left(N_{1}\right)$ & $N_{2}$ & & $\sigma\left(N_{2}\right)$ & & $N_{3}$ & & $\sigma(\Lambda$ & $\left.N_{3}\right)$ & $x^{2}$ & D.o.f. & $\mathrm{p}$ \\
\hline & -9.0 & -9.0 & 1.107 & $7515 \mathrm{e}-02$ & & $\overline{96899 \mathrm{e}-03}$ & $\begin{array}{ll}03 & -9.000000\end{array}$ & $0 \mathrm{e}+00$ & -9.000000 & $\mathrm{e}+00$ & -9.00000 & +00 & -9.0000 & $\overline{00 e+00}$ & 8.40 & 7.00 & \\
\hline & -9.0 & -9.0 & 1.747 & $7728 \mathrm{e}-02$ & 3.70 & 08279e-03 & $03-9.000000$ & $0 \mathrm{e}+00$ & -9.000000 & $e+00$ & -9.000000 & 00 & -9.0000 & $00 \mathrm{e}+00$ & 37.76 & 19.00 & \\
\hline & -9.0 & -9.0 & 8.883 & $3478 \mathrm{e}-04$ & 3.64 & 40030e-04 & $04-9.000000$ & $0 e+00$ & -9.000000 & $e+00$ & -9.000000 & $0 \mathrm{e}+00$ & -9.0000 & $000+00$ & 18.59 & 10.00 & $4.57 \xi$ \\
\hline-1 & -9.0 & -9.0 & 2.510 & $0829 \mathrm{e}-02$ & 4.82 & 22976e-03 & $03-9.000000$ & $0 \mathrm{e}+00$ & -9.000000 & $\mathrm{e}+00$ & -9.000000 & $0 \mathrm{e}+00$ & -9.0000 & $000 \mathrm{e}+00$ & 85.86 & 18.00 & \\
\hline-0.5 & -0.5 & -9.0 & 1.446 & $6221 \mathrm{e}-05$ & 1.24 & 48363e-06 & $06 \quad 2.000459$ & $9 e-04$ & $0.000000 \mathrm{e}$ & $e+00$ & -9.000000 & $0 \mathrm{e}+00$ & -9.0000 & $00 \mathrm{e}+00$ & 203.49 & 64.00 & $-3.484 \mathrm{e}-07$ \\
\hline
\end{tabular}

Notes. GRB: GRB name. Fil: filter name. Fit: fitting function. 0: $f(t)=N_{1} t^{-\alpha_{1}} ; 1: f(t)=N_{1}\left(\left(t / t_{b r, 1}\right)^{-\alpha_{1} / s_{1}}+\left(t / t_{\mathrm{br}, 2}\right)^{-\alpha_{2} / s_{1}}\right)^{s_{1}} ; 2: f(t)=$ $N_{1}\left(\left(t / t_{\mathrm{br}, 1}\right)^{-\alpha_{1} / s_{1}}+\left(t / t_{\mathrm{br}, 2}\right)^{-\alpha_{2} / s_{1}}\right)^{s_{1}}+N_{2}\left(\left(t / t_{\mathrm{br}, 3}\right)^{-\alpha_{3} / s_{2}}+\left(t / t_{\mathrm{br}, 4}\right)^{-\alpha_{4} / s_{2}}\right)^{s_{2}} ; 4: f(t)=N_{1}\left(\left(t / t_{\mathrm{br}, 1}\right)^{-\alpha_{1} / s_{1}}+\left(t / t_{\mathrm{br}, 2}\right)^{-\alpha_{2} / s_{1}}\right)^{s_{1}}+N_{2}(t)^{-\alpha_{3}} ; 6: f(t)=$ $N_{1}\left(\left(t / t_{\mathrm{br}, 1}\right)^{-\alpha_{1} / s_{1}}+\left(t / t_{\mathrm{br}, 2}\right)^{-\alpha_{2} / s_{1}}\right)^{s_{1}}+N_{2}(t)^{-\alpha_{3}} ; 8: f(t)=N_{1}\left(\left(t / t_{\mathrm{br}, 1}\right)^{-\alpha_{1} / s_{1}}+\left(t / t_{\mathrm{br}, 2}\right)^{-\alpha_{2} / s_{1}}\right)^{s_{1}}+N_{2}\left(\left(t / t_{\mathrm{br}, 3}\right)^{-\alpha_{3} / s_{2}}+\left(t / t_{\mathrm{br}, 4}\right)^{-\alpha_{4} / s_{2}}\right)^{s_{2}}+N_{3}\left(\left(t / t_{\mathrm{br}, 5}\right)^{-\alpha_{5} / s_{3}}+\right.$ $\left.\left(t / t_{\mathrm{br}, 6}\right)^{-\alpha_{6} / s_{3}}\right)^{s_{3}}$; 9: $f(t)=N_{1}\left(\left(t / t_{\mathrm{br}, 1}\right)^{-\alpha_{1} / s_{1}}+\left(t / t_{\mathrm{br}, 2}\right)^{-\alpha_{2} / s_{1}}\right)^{s_{1}}+N_{2}\left(\left(t / t_{\mathrm{br}, 3}\right)^{-\alpha_{3} / s_{2}}+\left(t / t_{\mathrm{br}, 4}\right)^{-\alpha_{4} / s_{2}}\right)^{s_{2}}+N_{3}(t)^{-\alpha_{5}}$. Ok: " 1 " denotes the data used for the comparison with the X-ray data and plotted in Figs. C.1-C.4 (available at the CDS). $T_{\min }\left(T_{\max }\right)$ : initial (end) time of the observations in rest frame (s). $t_{\mathrm{br}}, \sigma\left(t_{\mathrm{br}}\right)$ : trigger times with relative errors (s). $\alpha, \sigma(\alpha)$ : slopes with relative errors. $s$ : smoothness parameters. $N, \sigma N$ : normalization with relative error $(\mathrm{mJy}) \cdot \chi^{2}$ : chi-square. D.o.f.: degree of freedom. pval: p_value. A " -9 " indicates that the LC does not show this phase and the value of that parameter is therefore absent. This table is available in its entirety in a machine-readable form at the CDS (table1c.dat, ReadMe). A portion is shown here for guidance.

Table C.2. Parameters of the optical/X-ray SEDs fitted with a single power-law.

\begin{tabular}{lcccccccccccccccc}
\hline \hline GRB & Host & Part & $\beta_{\mathrm{op}, \mathrm{X}}$ & $\sigma\left(\beta_{\mathrm{op}, \mathrm{X}}\right)$ & $\mathrm{NH}$ & $\sigma(N H)$ & $E(B-V)$ & $\sigma(E(B-V))$ & $N$ & $\sigma(N)$ & $\chi^{2}$ & d.o.f. & p_val & $T_{\mathrm{i}}$ & $T_{\mathrm{f}}$ & ok \\
\hline 050319 & MW & $\mathrm{P}$ & 0.755 & 0.020 & -0.269 & 0.184 & 0.082 & 0.019 & 0.215 & 0.025 & $9.976 \mathrm{e}+01$ & 42 & $1.340 \mathrm{e}-06$ & 300. & 1500. & 0 \\
050319 & MW & $\mathrm{N}$ & 0.945 & 0.026 & -0.204 & 0.420 & 0.030 & 0.025 & 0.006 & 0.001 & $7.258 \mathrm{e}+01$ & 10 & $3.190 \mathrm{e}-07$ & 17000. & 300000. & 0 \\
050401 & MW & $\mathrm{P}$ & 0.873 & 0.075 & 1.840 & 0.322 & 0.667 & 0.082 & 1.830 & 0.851 & $3.331 \mathrm{e}+01$ & 146 & $1.000 \mathrm{e}+00$ & 200. & 2000. & 1 \\
050408 & $\mathrm{SMC}$ & $\mathrm{N}$ & 0.679 & 0.016 & 0.632 & 0.337 & 0.247 & 0.007 & 0.012 & 0.000 & $2.273 \mathrm{e}+01$ & 28 & $7.470 \mathrm{e}-01$ & 20000. & 40000. & 0 \\
$050416 \mathrm{~A}$ & LMC & $\mathrm{P}$ & 0.627 & 0.015 & 0.307 & 0.086 & 0.232 & 0.041 & 0.054 & 0.005 & $2.702 \mathrm{e}+01$ & 24 & $3.040 \mathrm{e}-01$ & 700. & 2000. & 0 \\
\hline
\end{tabular}

Notes. GRB: GRB name. Host: the chosen extinction law; Milky Way (MW), Large Magellanic Cloud (LMC), Small Magellanic Cloud (SMC). Part: X-ray LC part considered; S steep decay, P plateau, $\mathrm{N}$ normal decay. $\beta_{\mathrm{op}, X}, \sigma\left(\beta_{\mathrm{op}, X}\right)$ : spectral index with error. NH, $\sigma(\mathrm{NH})$ : hydrogen column density with relative error $\left(10^{22} \mathrm{~cm}^{-2}\right) . E(B-V), \sigma(E(B-V))$ : optical absorption with relative error $(\mathrm{mag})$. $\mathrm{N}, \sigma(\mathrm{N})$ : normalization with error $(\mathrm{mJy})$. $\chi^{2}$ : chi-square. d.o.f.: degree of freedom. pval: $\mathrm{p}_{-}$value. $T_{\mathrm{i}}, T_{\mathrm{f}}$ : initial (end) time of the interval of the SED in observer time (s). Ok: 1 if the best fitting function is a power-law, otherwise 0 . This table is available in its entirety in a machine-readable form at the CDS (table2c.dat, ReadMe). A portion is shown here for guidance. 
Table C.3. Parameters of the optical/X-ray SEDs fitted with a broken power-law.

\begin{tabular}{|c|c|c|c|c|c|c|c|c|c|c|}
\hline GRB & Host & Part & $\beta_{\text {op }}$ & $\sigma\left(\beta_{\mathrm{op}}\right)$ & $\beta_{\mathrm{X}}$ & $\sigma\left(\beta_{\mathrm{X}}\right)$ & $v_{\mathrm{br}}$ & $\sigma\left(v_{\mathrm{br}}\right)$ & $\mathrm{NH}$ & $\sigma(\mathrm{NH})$ \\
\hline 050319 & MW & $\mathrm{P}$ & 0.358 & 0.000 & 0.858 & 0.077 & 1.271 & 1.198 & -0.030 & 0.283 \\
\hline 050319 & MW & $\mathrm{N}$ & 0.607 & 0.000 & 1.107 & 0.045 & 0.545 & 0.118 & 0.114 & 0.437 \\
\hline 050401 & SMC & $\mathrm{P}$ & 0.372 & 0.000 & 0.872 & 0.146 & 0.455 & 87.600 & 1.850 & 2.540 \\
\hline 050408 & LMC & $\mathrm{N}$ & 0.342 & 0.000 & 1.210 & 0.310 & 22.350 & 26.190 & 1.560 & 0.690 \\
\hline 050416A & SMC & $\mathrm{P}$ & 0.619 & 0.000 & 0.951 & 0.156 & 339.900 & 131.310 & 0.398 & 0.097 \\
\hline$E(B-V)$ & $\sigma(E(B-V))$ & $N$ & $\sigma(N)$ & $\chi^{2}$ & d.o.f. & p_val & $T_{\mathrm{i}}$ & $T_{\mathrm{f}}$ & ok & \\
\hline 0.103 & 0.022 & 0.327 & 0.048 & $4.050 \mathrm{e}+01$ & 40 & $4.467 \mathrm{e}-01$ & 300.000 & 1500.000 & 1 & \\
\hline 0.165 & 0.034 & 0.021 & 0.005 & $4.050 \mathrm{e}+01$ & 9 & $3.770 \mathrm{e}-05$ & 17000.000 & 300000.000 & 1 & \\
\hline 0.462 & 10.500 & 2.700 & 263.000 & $2.050 \mathrm{e}+02$ & 145 & $7.460 \mathrm{e}-04$ & 200.000 & 2000.000 & 0 & \\
\hline 0.290 & 0.000 & 0.020 & 0.001 & $2.654 \mathrm{e}+01$ & 27 & $4.885 \mathrm{e}-01$ & 20000.000 & 40000.000 & 1 & \\
\hline 0.321 & 0.044 & 0.060 & 0.005 & $1.890 \mathrm{e}+01$ & 23 & $7.059 \mathrm{e}-01$ & 700.000 & 2000.000 & 1 & \\
\hline
\end{tabular}

Notes. GRB: GRB name. Host: galaxy chosen as model to fit the SED; Milky Way (MW), Large Magellanic Cloud (LMC), Small Magellanic Cloud (SMC). Part: X-ray LC part considered; "S" steep decay, "P" plateau, "N" normal decay. $\beta_{\text {op }}, \sigma\left(\beta_{\text {op }}\right)$ : optical spectral index with error. $\beta_{\mathrm{X}}$, $\sigma\left(\beta_{\mathrm{X}}\right)$ : X-ray spectral index with error. $v, \sigma(v)$ : observer frame break frequency $\left(10^{15} \mathrm{~Hz}\right) . \mathrm{NH}, \sigma(N H)$ : hydrogen column density with relative error $\left(10^{22} \mathrm{~cm}^{-2}\right) . E(B-V), \sigma(E(B-V))$ : optical absorption with relative error $(\mathrm{mag}) . N, \sigma(N)$ : normalization with error (mJy). $\chi^{2}$ : chi-square. d.o.f.: degree of freedom. p_val: p_value. $T_{\mathrm{i}}, T_{\mathrm{f}}$ : initial (end) time of the interval of the SED in observer time (s). Ok: "1" if the best fitting function is a broken power-law, otherwise " 0 ". This table is available in its entirety in a machine-readable form at the CDS (table3c.dat, ReadMe). A portion is shown here for guidance.

Table C.4. Parameters of the X-ray spectrum fit.

\begin{tabular}{lcccccccc}
\hline \hline GRB & $N$ & $\sigma(N)$ & $\beta$ & $\sigma(\beta)$ & $\mathrm{NH}$ & $\sigma(\mathrm{NH})$ & $T_{\mathrm{i}}$ & $T_{\mathrm{f}}$ \\
\hline 050319 & 0.365 & 0.170 & 0.857 & 0.077 & -0.005 & 0.284 & 300.000 & 1500.000 \\
050319 & 0.006 & 0.009 & 0.941 & 0.246 & -0.310 & 0.823 & 17000.000 & 300000.000 \\
050401 & 1.719 & 0.790 & 0.864 & 0.075 & 2.000 & 0.334 & 200.000 & 2000.000 \\
050408 & 0.345 & 0.686 & 1.200 & 0.314 & 1.620 & 0.692 & 20000.000 & 40000.000 \\
$050416 \mathrm{~A}$ & 1.159 & 1.082 & 1.104 & 0.146 & 0.791 & 0.168 & 700.000 & 2000.000 \\
\hline
\end{tabular}

Notes. GRB: GRB name. $N, \sigma(N)$ : normalization with error $(\mathrm{keV}) . \beta, \sigma(\beta)$ : spectral index. $\mathrm{NH}, \sigma(\mathrm{NH})$ : hydrogen column density with error $\left(10^{22} \mathrm{~cm}^{-2}\right) . T_{\mathrm{i}}, T_{\mathrm{f}}$ : initial (end) time of the interval of the SED in observer time (s). This table is available in its entirety in a machine-readable form at the CDS (table4c.dat, ReadMe). A portion is shown here for guidance. 
E. Zaninoni et al.: The gamma-ray burst optical light-curve zoo: comparison with the X-ray observations

Table C.5. Information on the GRBs in our sample.

\begin{tabular}{lcc}
\hline \hline GRB & $z$ & DL \\
\hline 050319 & 3.240 & 28.369 \\
050401 & 2.900 & 24.806 \\
050408 & 1.236 & 8.644 \\
$050416 \mathrm{~A}$ & 0.653 & 3.913 \\
$050525 \mathrm{~A}$ & 0.606 & 3.567 \\
\hline
\end{tabular}

Notes. GRB: GRB name. $z$ : redshift. DL: luminosity distance (Gpc). Redshifts and luminosity distances are taken from M13. This table is available in its entirety in a machine-readable form at the CDS (table5c.dat, ReadMe). A portion is shown here for guidance.

Table C.6. Optical data used in the SED.

\begin{tabular}{lccccc}
\hline \hline GRB & Filter & $\lambda_{\mathrm{c}}$ & $F W H M$ & $F$ & $\sigma(F)$ \\
\hline 050319 & $b$ & 4392 & 975 & $1.029 \mathrm{e}-01$ & $1.229 \mathrm{e}-02$ \\
050319 & $v$ & 5468 & 769 & $2.144 \mathrm{e}-01$ & $1.297 \mathrm{e}-02$ \\
050319 & $R C$ & 6588 & 1568 & $2.184 \mathrm{e}-01$ & $3.237 \mathrm{e}-03$ \\
050319 & $C R$ & 6588 & 1568 & $2.901 \mathrm{e}-01$ & $8.830 \mathrm{e}-03$ \\
050319 & $v$ & 5468 & 769 & $8.072 \mathrm{e}-03$ & $8.283 \mathrm{e}-04$ \\
\hline
\end{tabular}

Notes. GRB: GRB name. Fil: filter. $\lambda_{\mathrm{c}}$ : central wavelength of the filter $(\AA)$. $F W H M$ : full width half maximum of the filter $(\AA) . F, \sigma(F)$ : flux with error $(\mathrm{mJy})$. This table is available in its entirety in a machinereadable form at the CDS (table6c.dat, ReadMe). A portion is shown here for guidance.
Table C.7. Conversion factors used to convert magnitude into flux density (Jy).

\begin{tabular}{|c|c|c|c|c|}
\hline Filter & $\lambda_{\mathrm{c}}(\AA)$ & $F W H M(\AA)$ & $F_{0}(\mathrm{Jy})$ & Reference \\
\hline$J$ & 12350 & 1620 & 1594 & (1) \\
\hline$H$ & 16620 & 2510 & 1024 & (1) \\
\hline$K S$ & 21590 & 2620 & 666.7 & (1) \\
\hline$v$ & 5468 & 769 & 3647 & (2) \\
\hline$b$ & 4392 & 975 & 4058 & (2) \\
\hline$u$ & 3465 & 785 & 1423 & (2) \\
\hline$u v w 1$ & 2600 & 693 & 901 & (2) \\
\hline uvm 2 & 2246 & 493 & 767 & (2) \\
\hline$u v w 2$ & 1928 & 657 & 716 & (2) \\
\hline white & 3850 & 2600 & 1732 & (2) \\
\hline$U_{J}$ & 3652 & 526 & 1890 & (3) \\
\hline$B_{J}$ & 4448 & 1008 & 4020 & (3) \\
\hline$V_{J}$ & 5505 & 827 & 3590 & (3) \\
\hline$R_{C}$ & 6588 & 1568 & 3020 & (3) \\
\hline$I_{C}$ & 8060 & 1542 & 2380 & (3) \\
\hline$u^{\prime}$ & 3585 & 556 & 1568.5 & (4) \\
\hline$g^{\prime}$ & 4858 & 1297 & 3965.9 & (4) \\
\hline$r^{\prime}$ & 6290 & 1358 & 3162.0 & (4) \\
\hline$i^{\prime}$ & 7706 & 1547 & 2602.0 & (4) \\
\hline$z^{\prime}$ & 9222 & 1530 & 2244.7 & (4) \\
\hline$g_{\text {Gunn }}$ & 5200 & 1400 & 3730 & (5) \\
\hline$r_{\text {Gunn }}$ & 6700 & 1400 & 4490 & (5) \\
\hline$i_{\text {Gunn }}$ & 7900 & 1600 & 4760. & (5) \\
\hline$z_{\text {Gunn }}$ & 9100 & 1300 & 4810 & (5) \\
\hline$U_{\text {Bessel }}$ & 3605.1 & 3031 & 1803.1 & (4) \\
\hline$B_{\text {Bessel }}$ & 4400.0 & 3701 & 4000.0 & (4) \\
\hline$V_{\text {Bessel }}$ & 5512.1 & 4733 & 3579.8 & (4) \\
\hline$R_{\text {Bessel }}$ & 6585.9 & 5504 & 2971.4 & (4) \\
\hline$I_{\text {Bessel }}$ & 8059.9 & 7042 & 2405.3 & (4) \\
\hline
\end{tabular}

References. (1) http://www.ipac.caltech.edu/2mass/ releases/allsky/faq.html\#jansky; (2) UVOT calibration files; (3) Fukugita et al. (1995); (4) http://svo.cab.inta-csic. es/; (5) Schneider et al. (1983). 


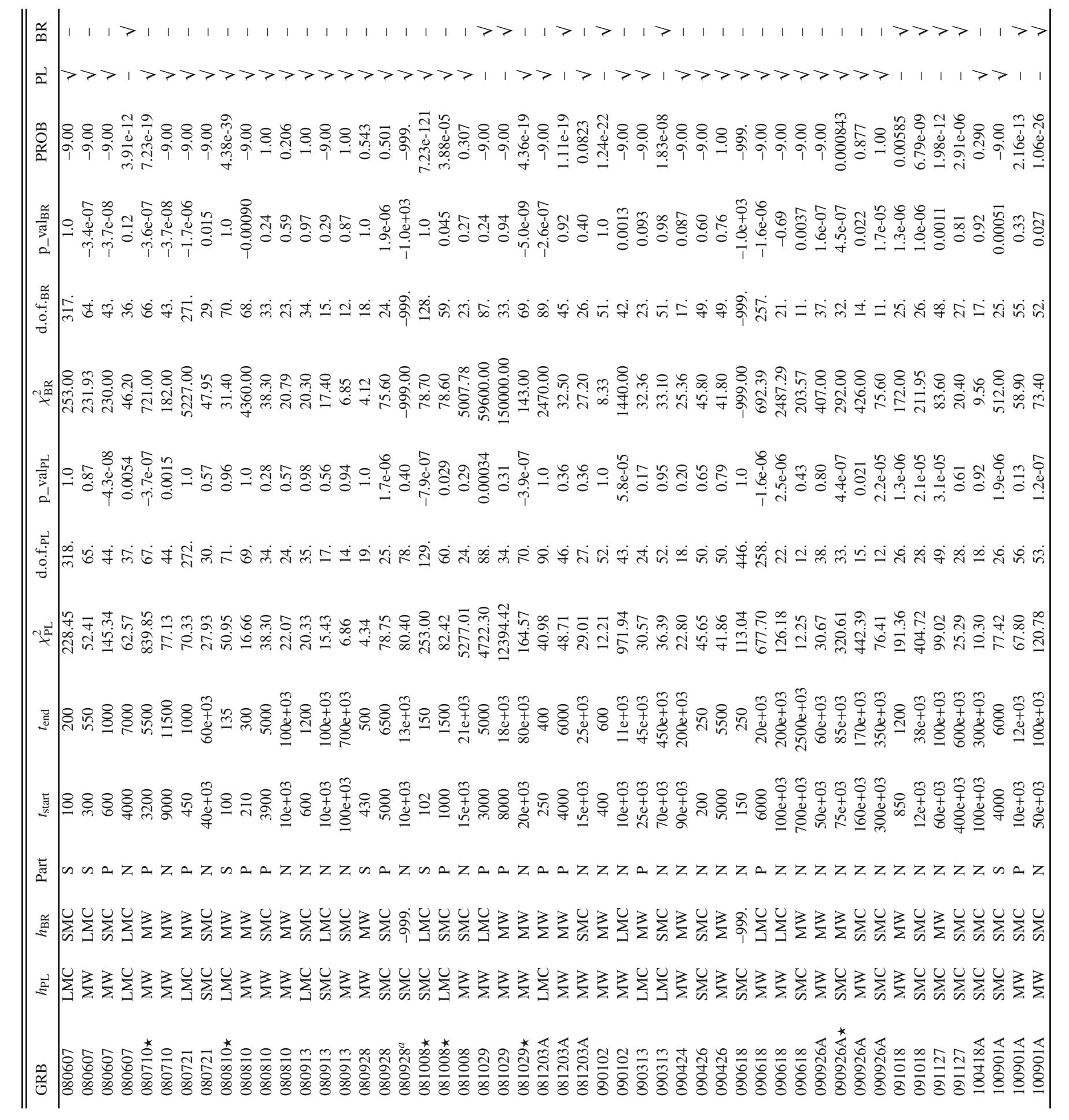


E. Zaninoni et al.: The gamma-ray burst optical light-curve zoo: comparison with the X-ray observations

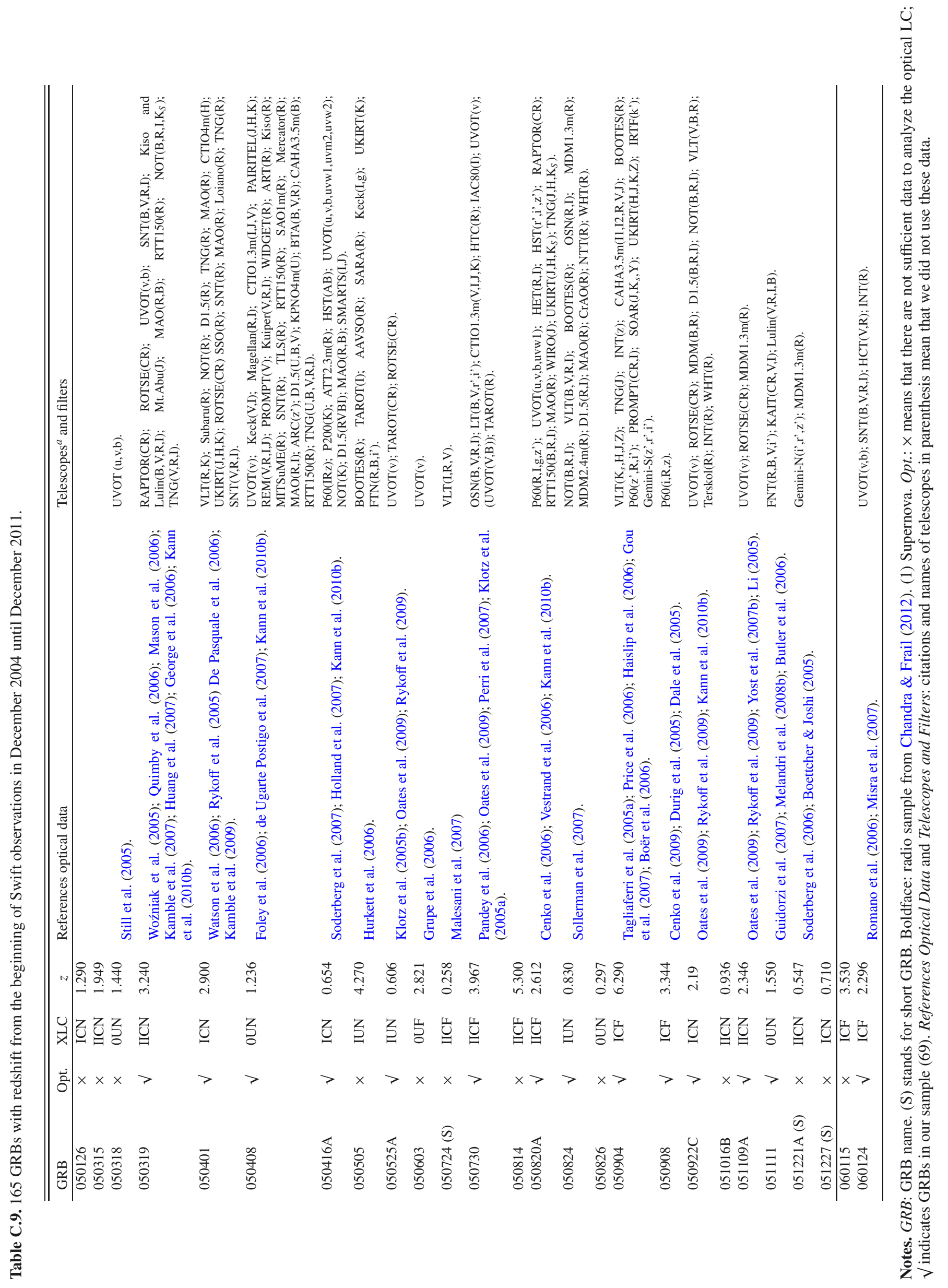




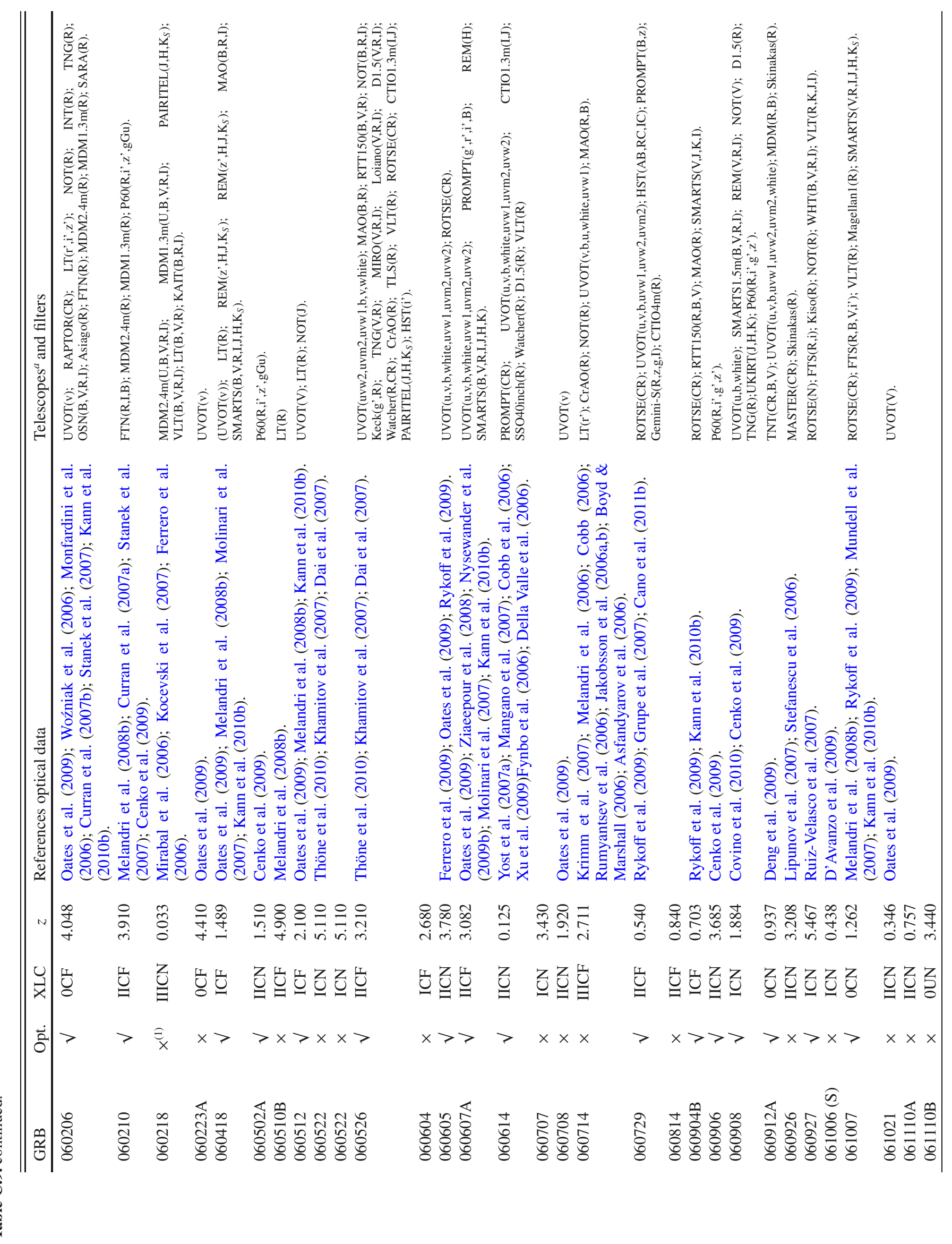


E. Zaninoni et al.: The gamma-ray burst optical light-curve zoo: comparison with the X-ray observations

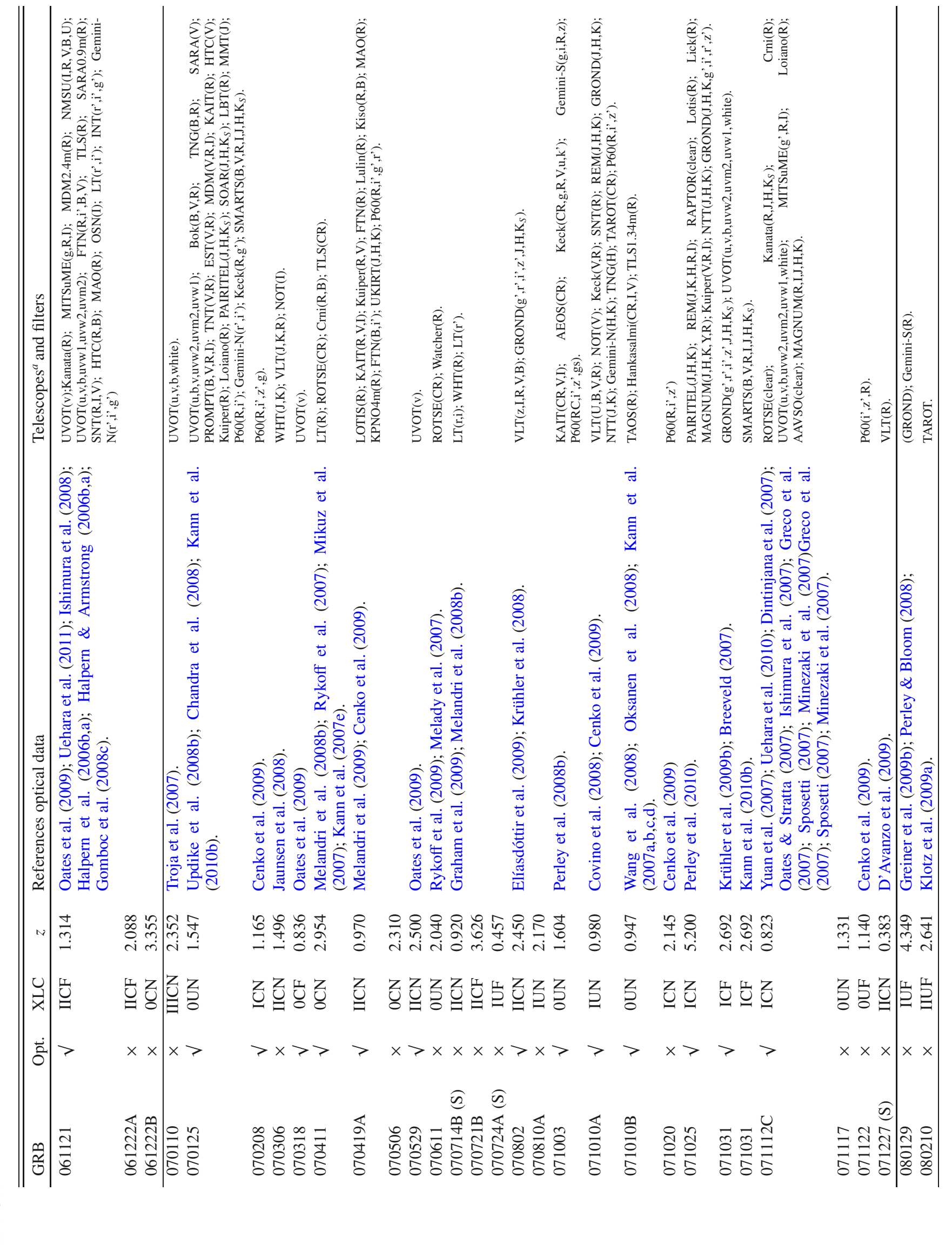

A12, page 31 of 55 


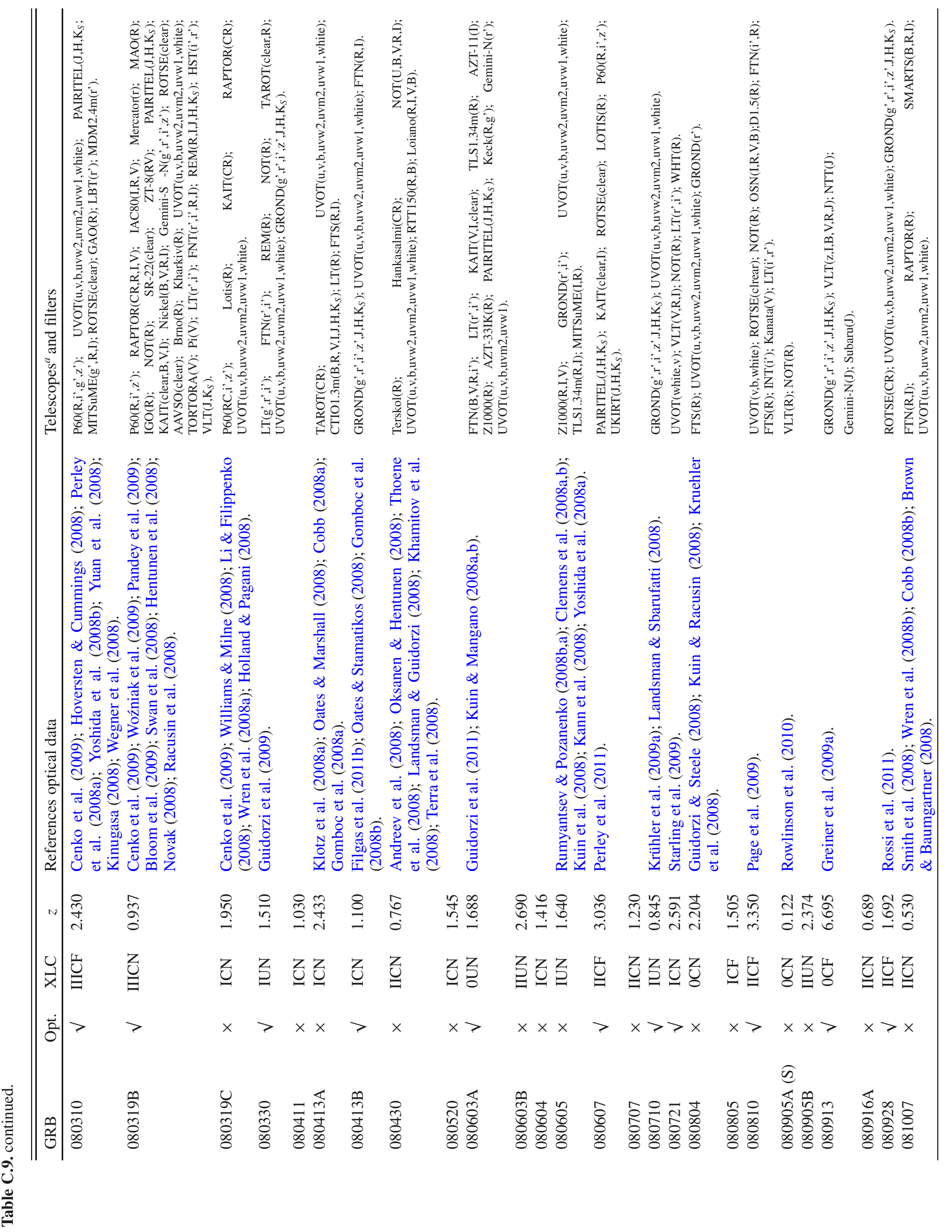




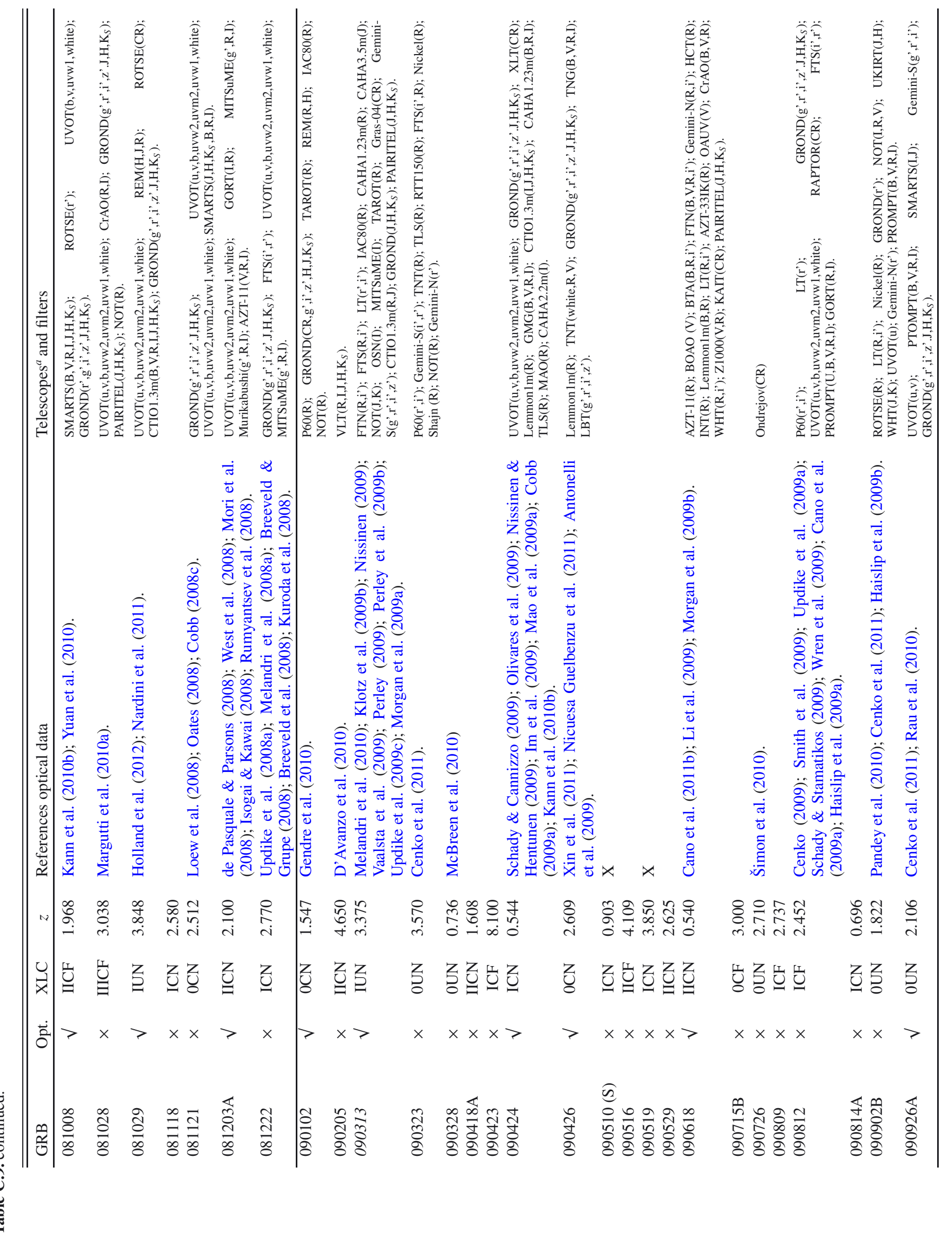




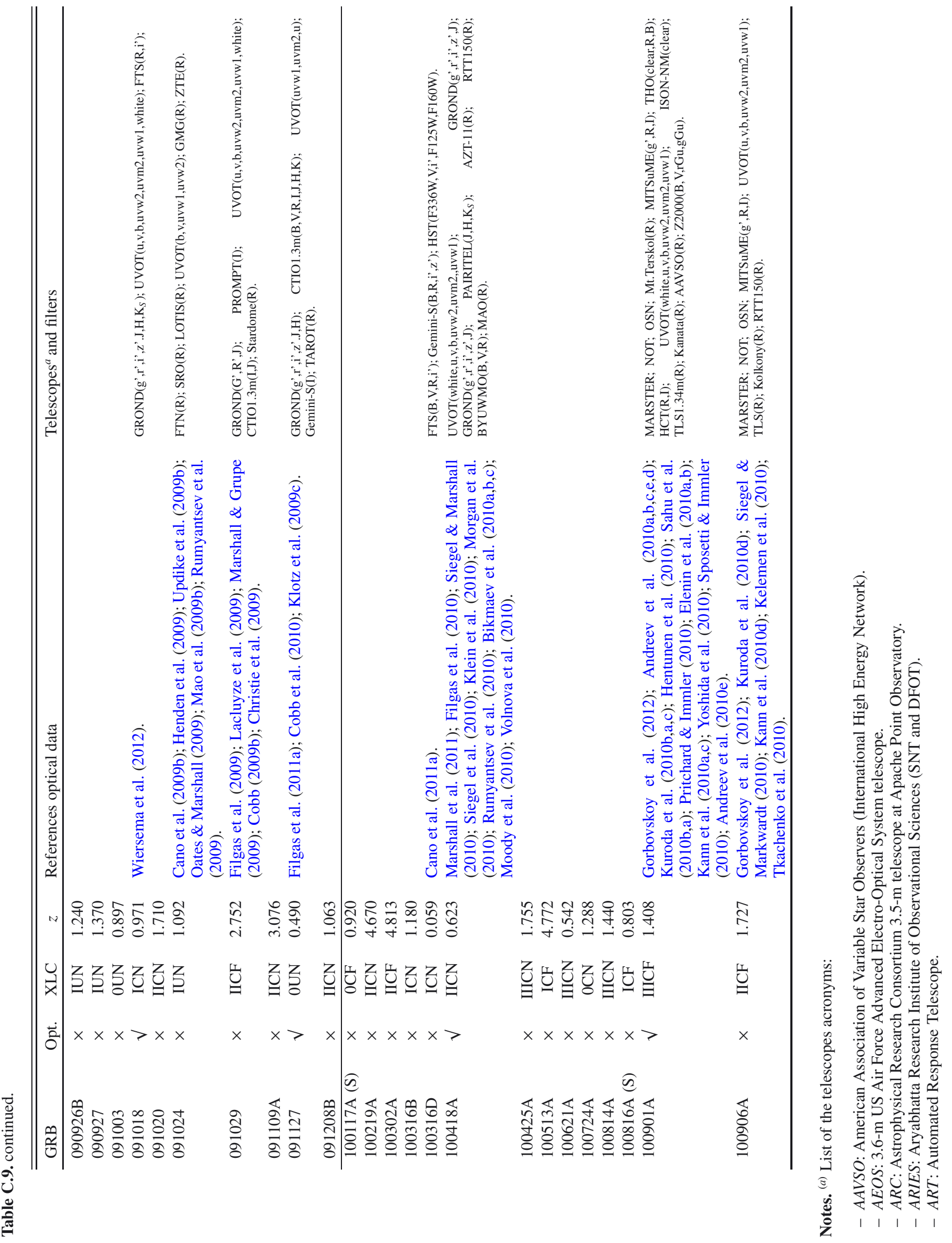




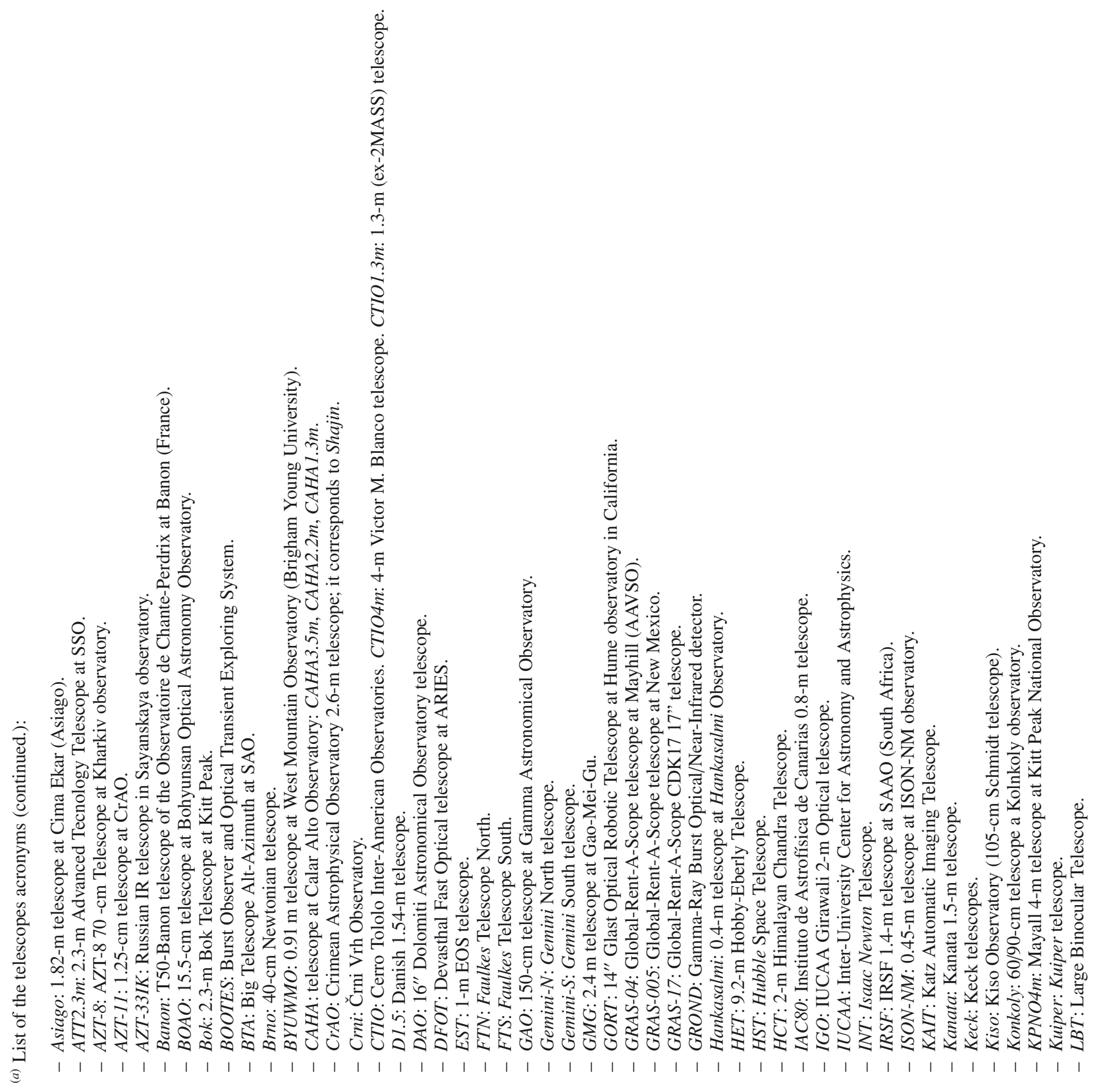




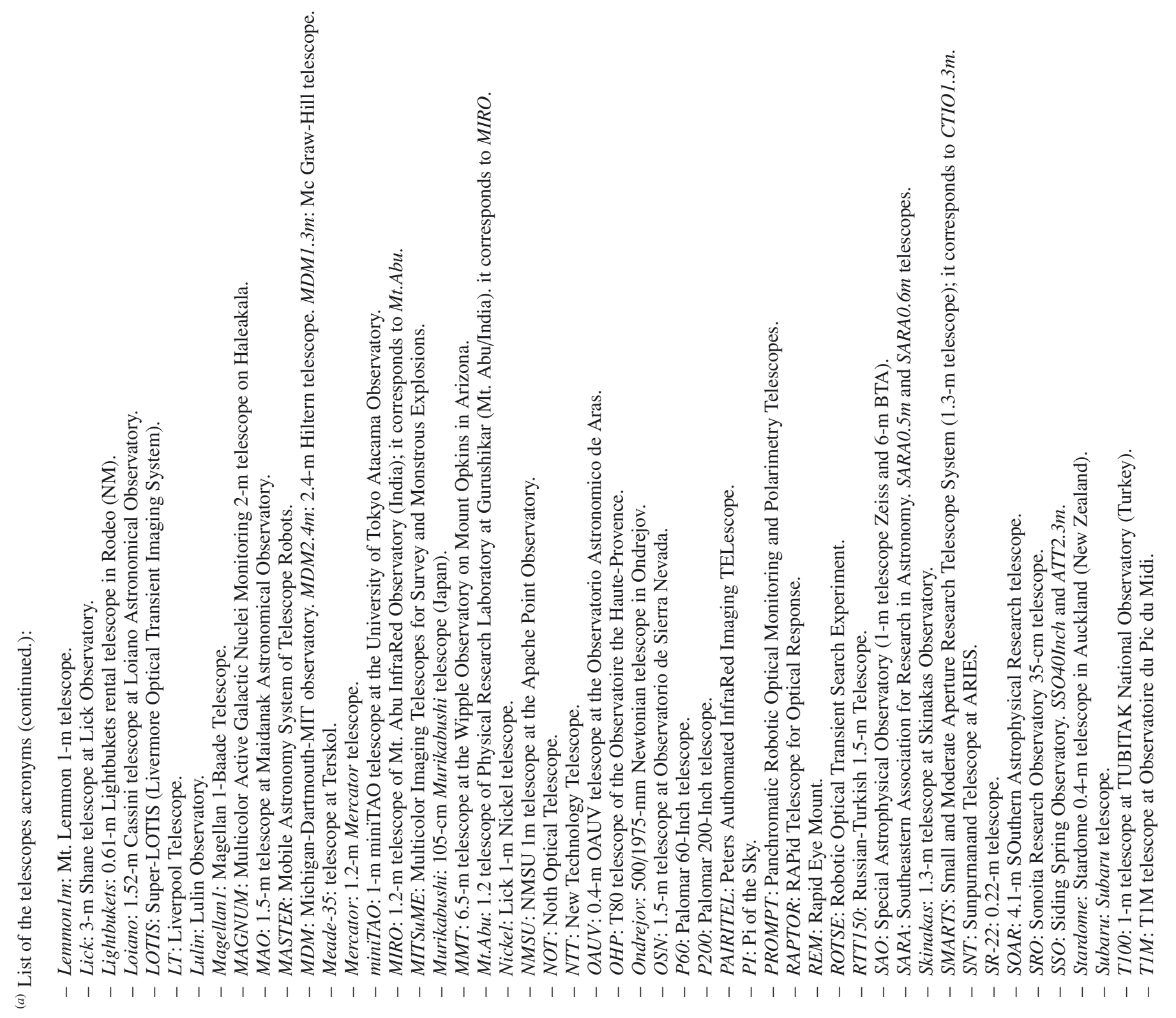


E. Zaninoni et al.: The gamma-ray burst optical light-curve zoo: comparison with the X-ray observations

(a) List of the telescopes acronyms (continued.):

- TAOS (A,B,C,D): Taiwanese-American Occultation Survey Telescopes.

- TAROT: Télescopes à Action Rapide pour les Objects Transitoires.

- Terskol: Zeiss-600 of Mt. (Pik) Terskol observatory.

- THO: Taurus Hill Observatory.

- TLS: Thüringer Landessternwarte Tauteburg. TLS2m and TLS1.34m.

- TNG: Telescopio Nazionale Galileo.

- TNT: 0.8-m Tsinghua-NOAC Telescope.

- TORTORA: camera mounted on REM telescope.

- UKIRT: United Kingdom Infra-Red Telescope.

- UVOT: Ultra-Violet/Optical Telescope on board Swift satellite.

- VLT: Very Large Telescope.

- Watcher: Watcher telescope.

- WHT: 4.2-m William Herchel Telescope.

- WIDGET: WIDe-field camera for Gamma-ray bursts Early Timing.

- WIRO: 2.3m Wyoming Infrared Observatory telescope in Wyoming (USA).

- XLT: 0.35-m C14 XLT telescope at Taurus Hill Observatory.

- Z1000: 1-m Zeiss-telescope at CrAO.

- Z2000: telescope at Mt. Terskol.

- Zadko: 1-m Zadko telescope.

- ZTE: 1.25-m ZTE telescope at the Crimean station of the Sternberg Astronomical Institute. 
A\&A 557, A12 (2013)
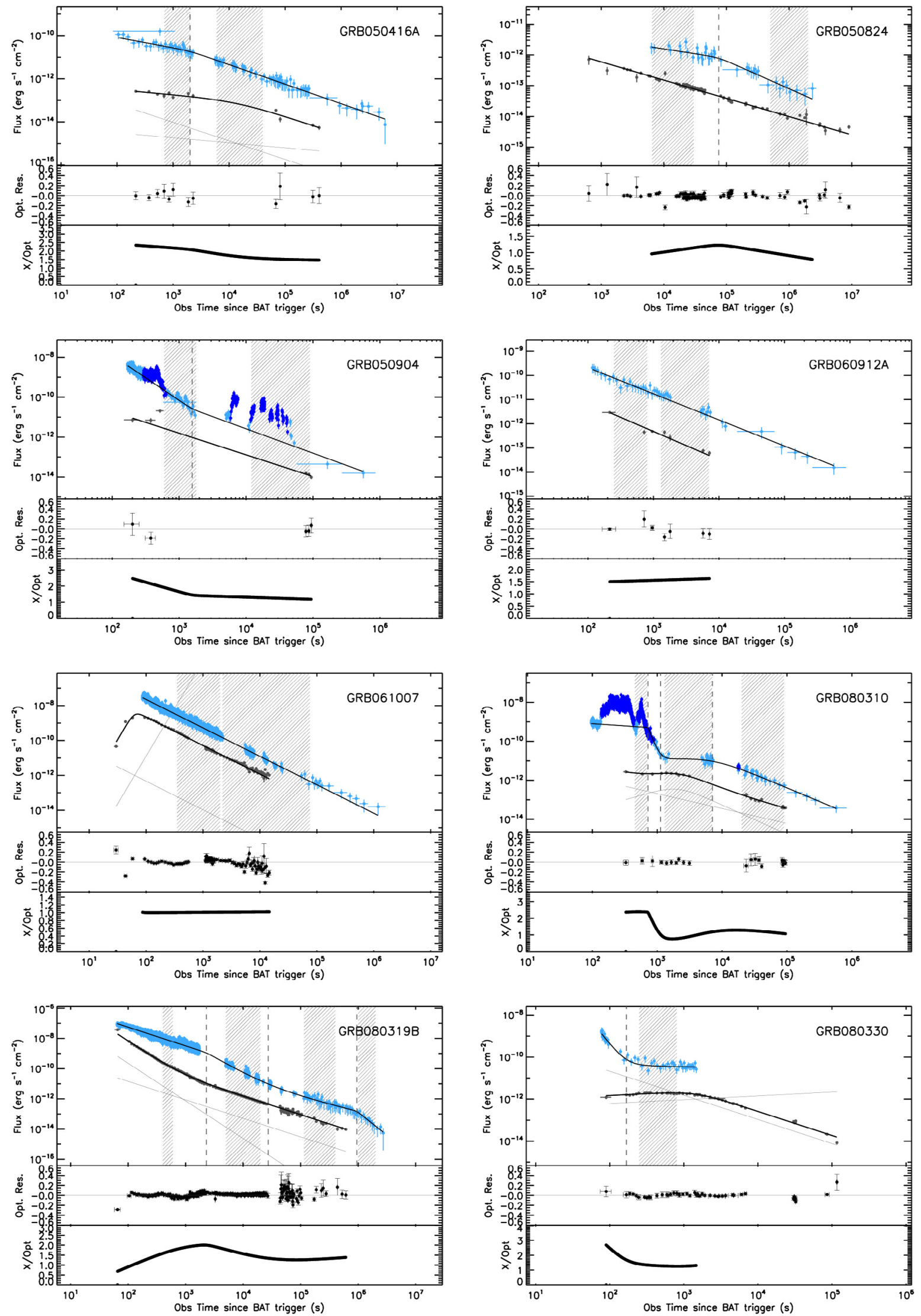

Fig. C.1. Comparison between optical and X-ray LCs. Top. Colored points: X-ray data. Dark color represents the excesses and light colors the continuum as calculated in M 13. Group A: blue/lightblue. Group B: red/orange. Group C: purple/magenta. Gray points: optical data. Black solid line: fit of the data. Gray solid line: components of the fit function used to fit the optical data. Middle. Ratio between the optical data and their fit function. The points have different colors when the optical data come from different filters. Bottom. Ratio between the X-ray flux and the optical flux. Hashed gray boxes: SED time intervals. 
E. Zaninoni et al.: The gamma-ray burst optical light-curve zoo: comparison with the X-ray observations
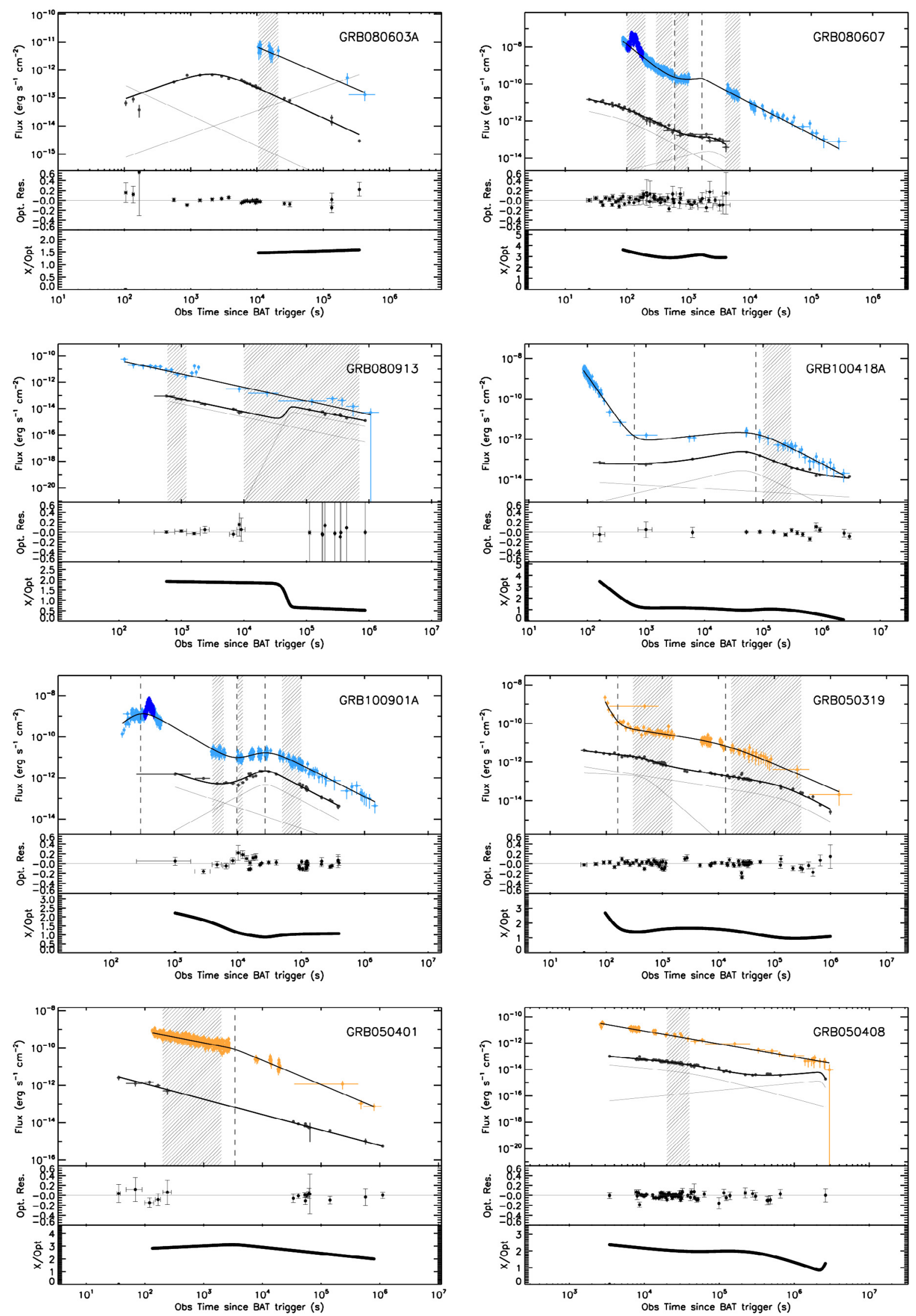

Fig. C.2. Comparison between optical and X-ray LCs. Color-coding as in Fig. C.1. 
A\&A 557, A12 (2013)
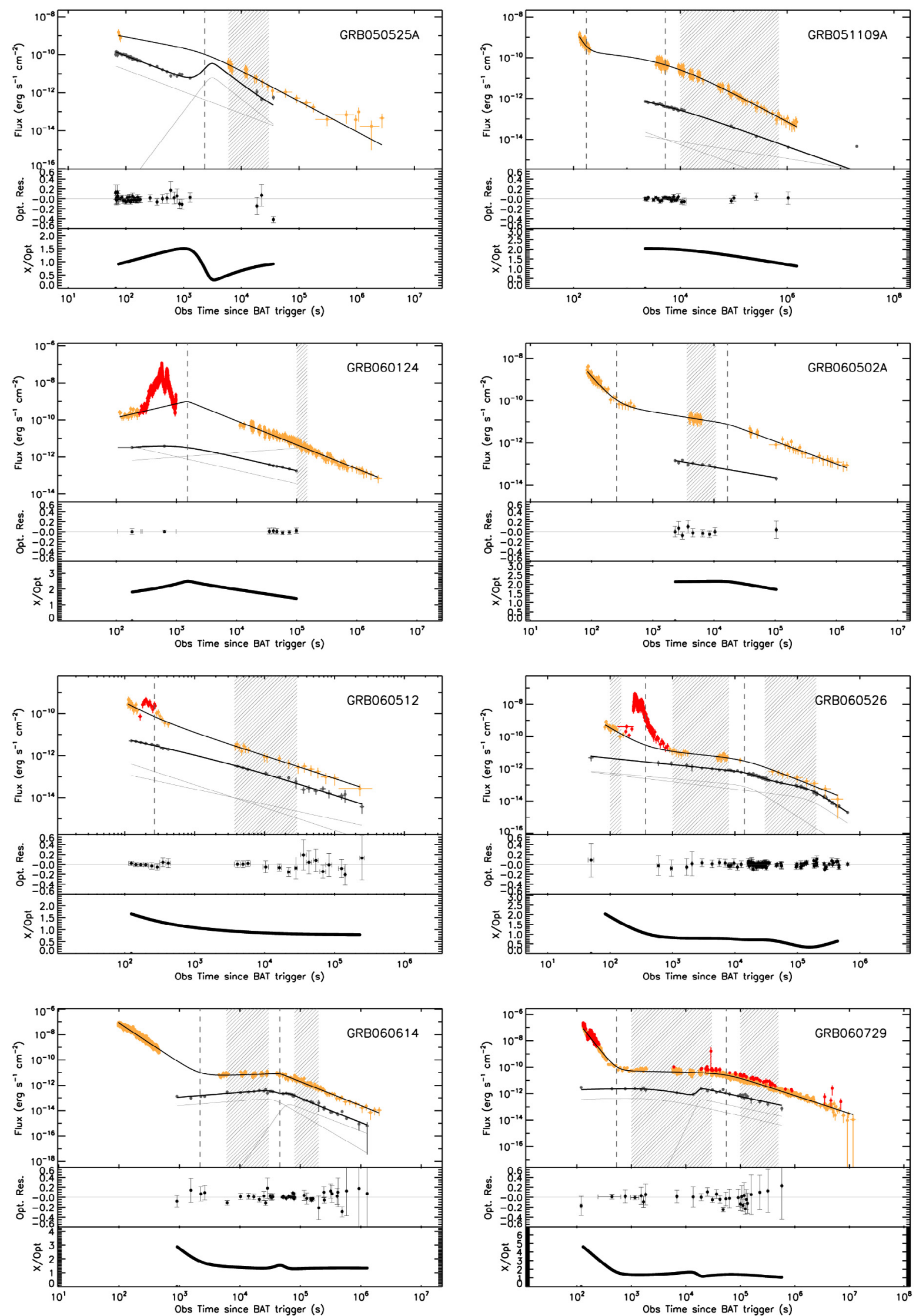

Fig. C.3. Comparison between optical and X-ray LCs. Color-coding as in Fig. C.1. 
E. Zaninoni et al.: The gamma-ray burst optical light-curve zoo: comparison with the X-ray observations
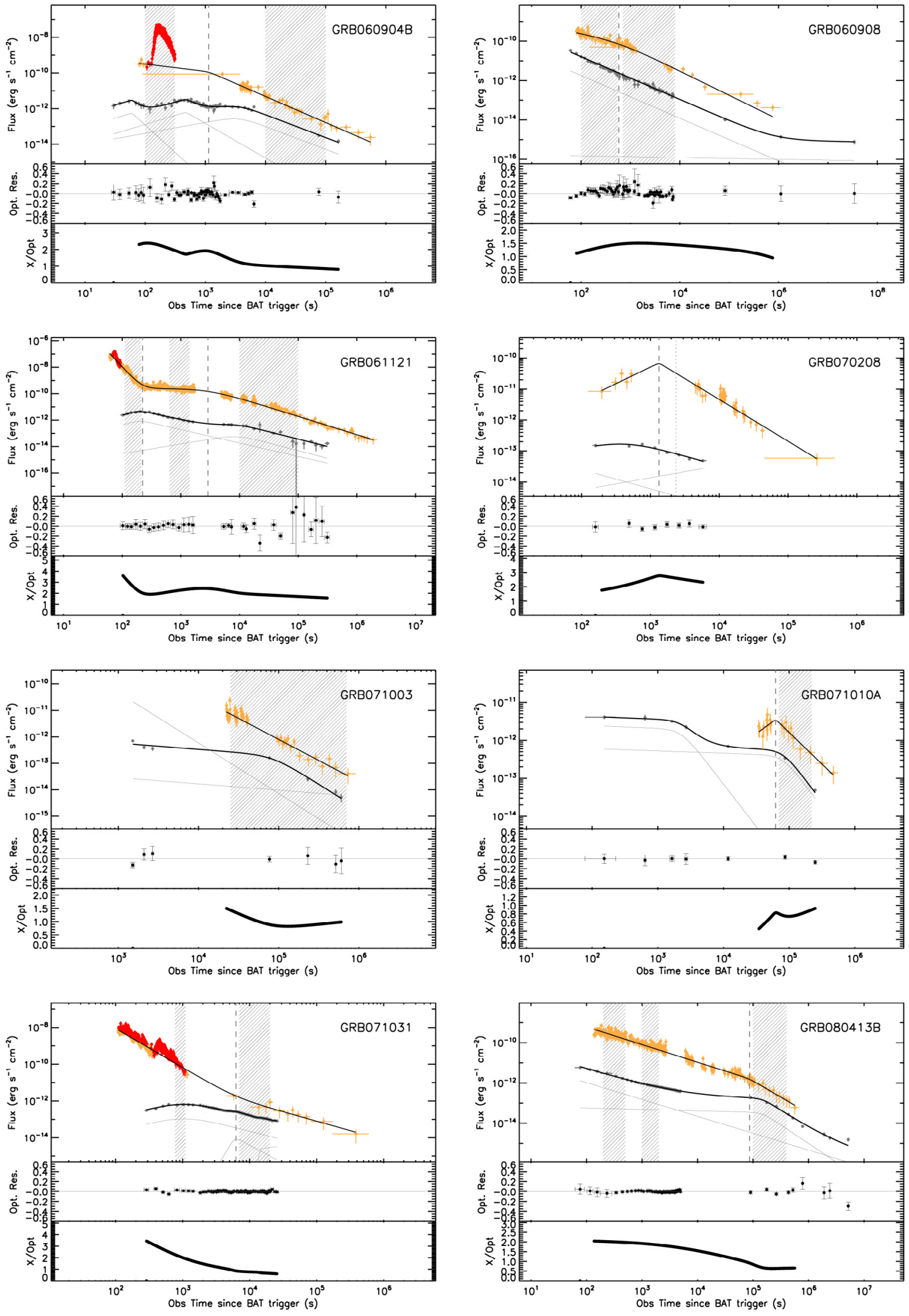

Fig. C.4. Comparison between optical and X-ray LCs. Color-coding as in Fig. C.1. 
A\&A 557, A12 (2013)
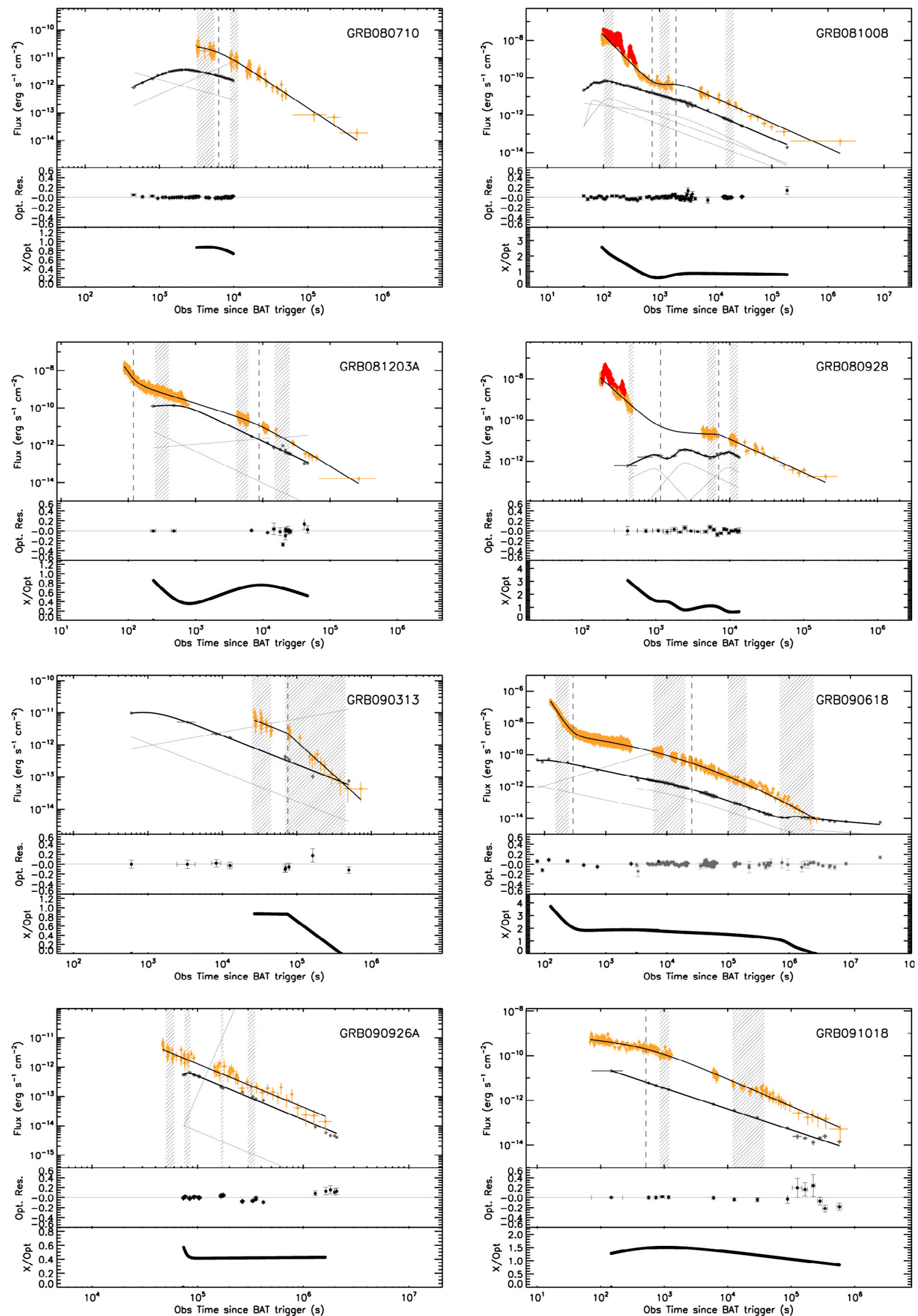

Fig. C.5. Comparison between optical and X-ray LCs. Color-coding as in Fig. C.1. 
E. Zaninoni et al.: The gamma-ray burst optical light-curve zoo: comparison with the X-ray observations
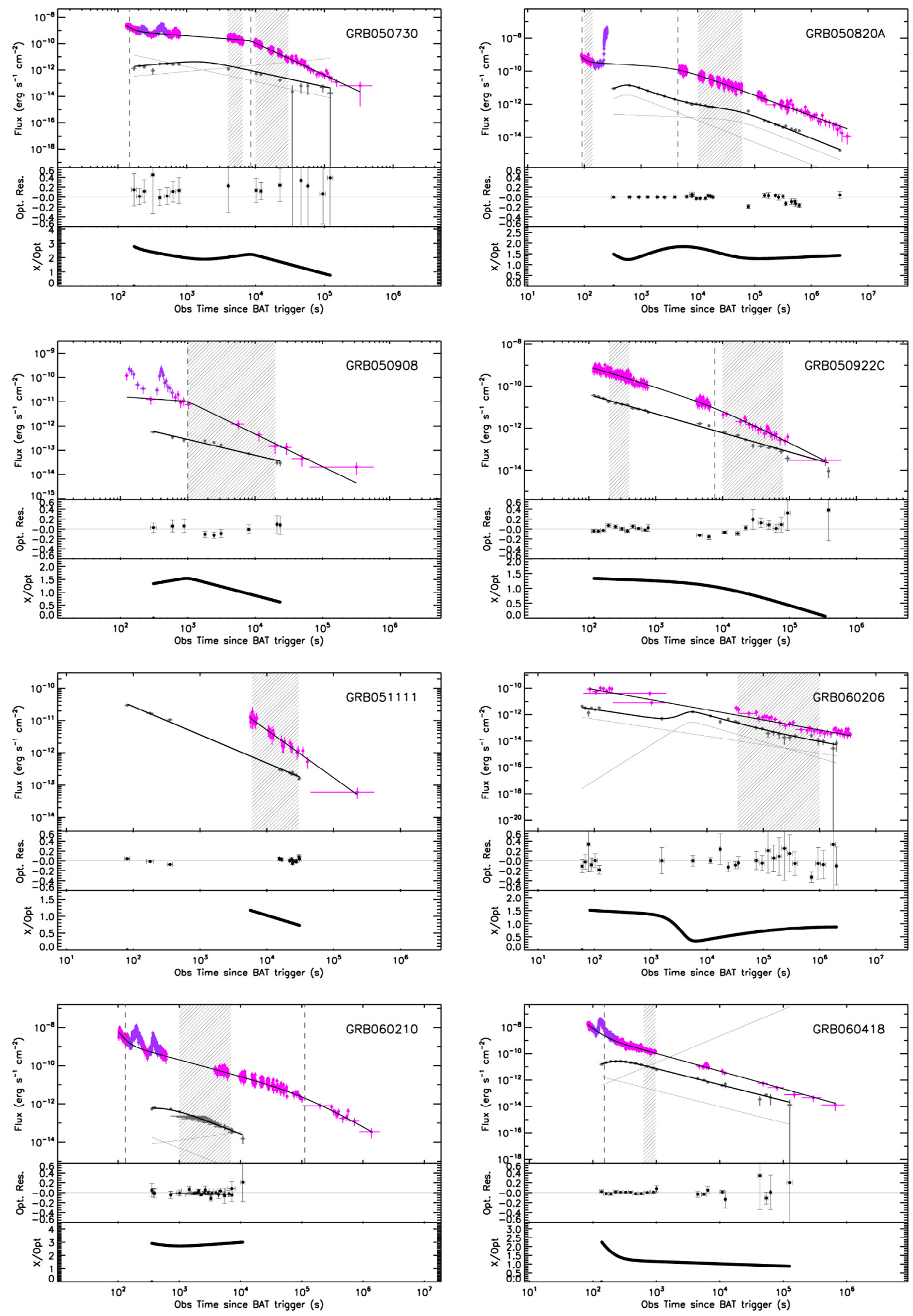

Fig. C.6. Comparison between optical and X-ray LCs. Color-coding as in Fig. C.1. 
A\&A 557, A12 (2013)
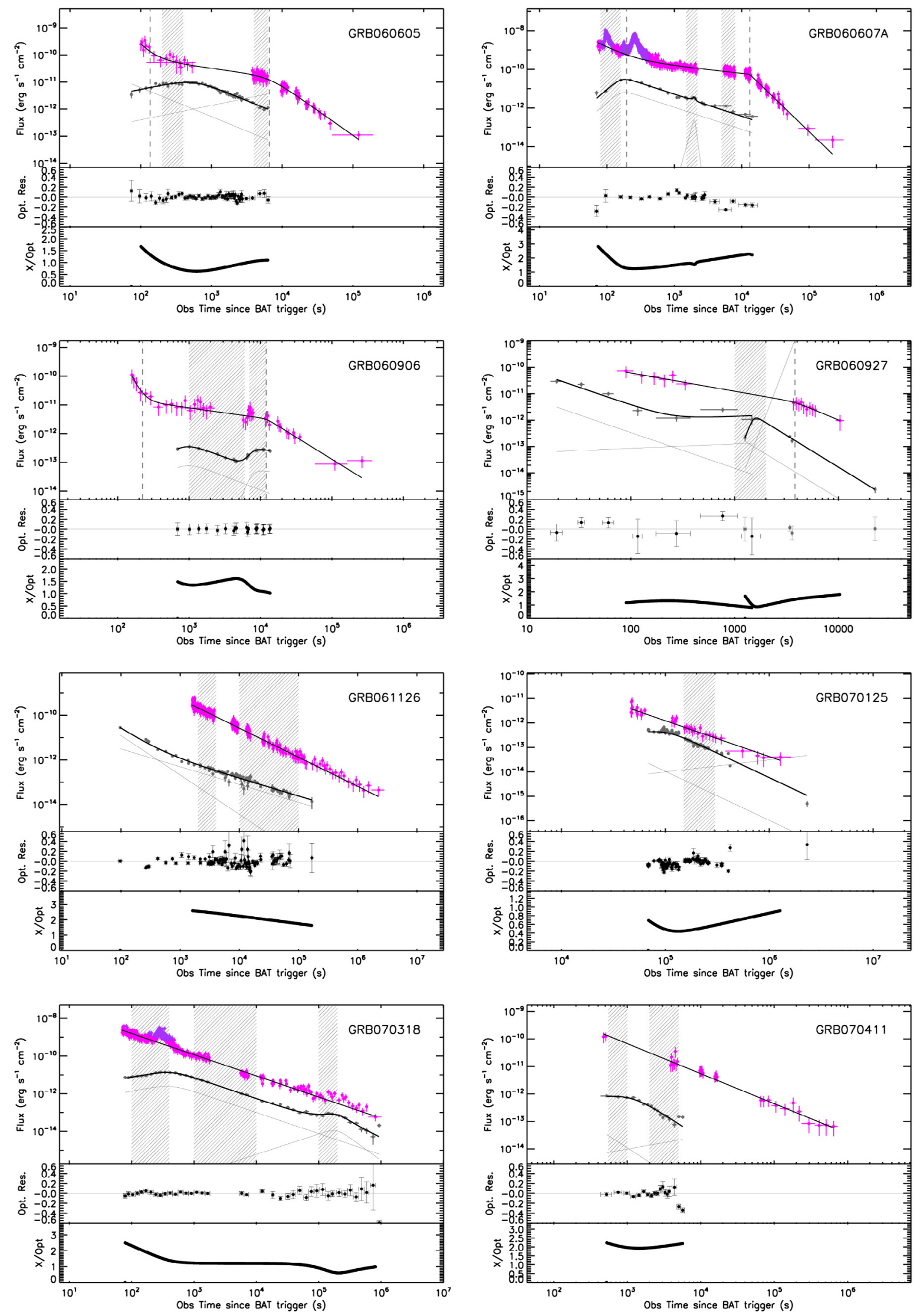

Fig. C.7. Comparison between optical and X-ray LCs. Color-coding as in Fig. C.1. 
E. Zaninoni et al.: The gamma-ray burst optical light-curve zoo: comparison with the X-ray observations
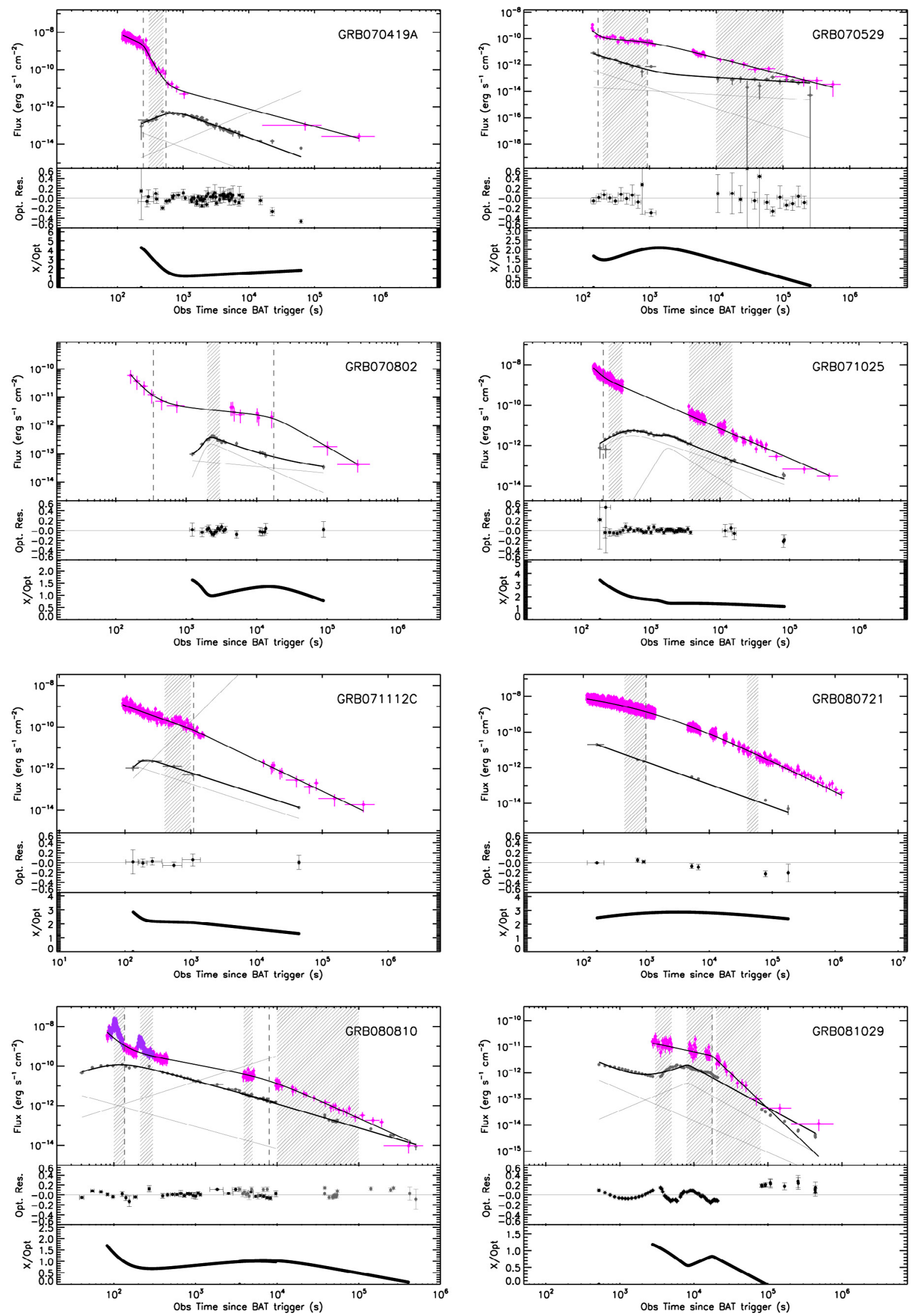

Fig. C.8. Comparison between optical and X-ray LCs. Color-coding as in Fig. C.1. 
A\&A 557, A12 (2013)
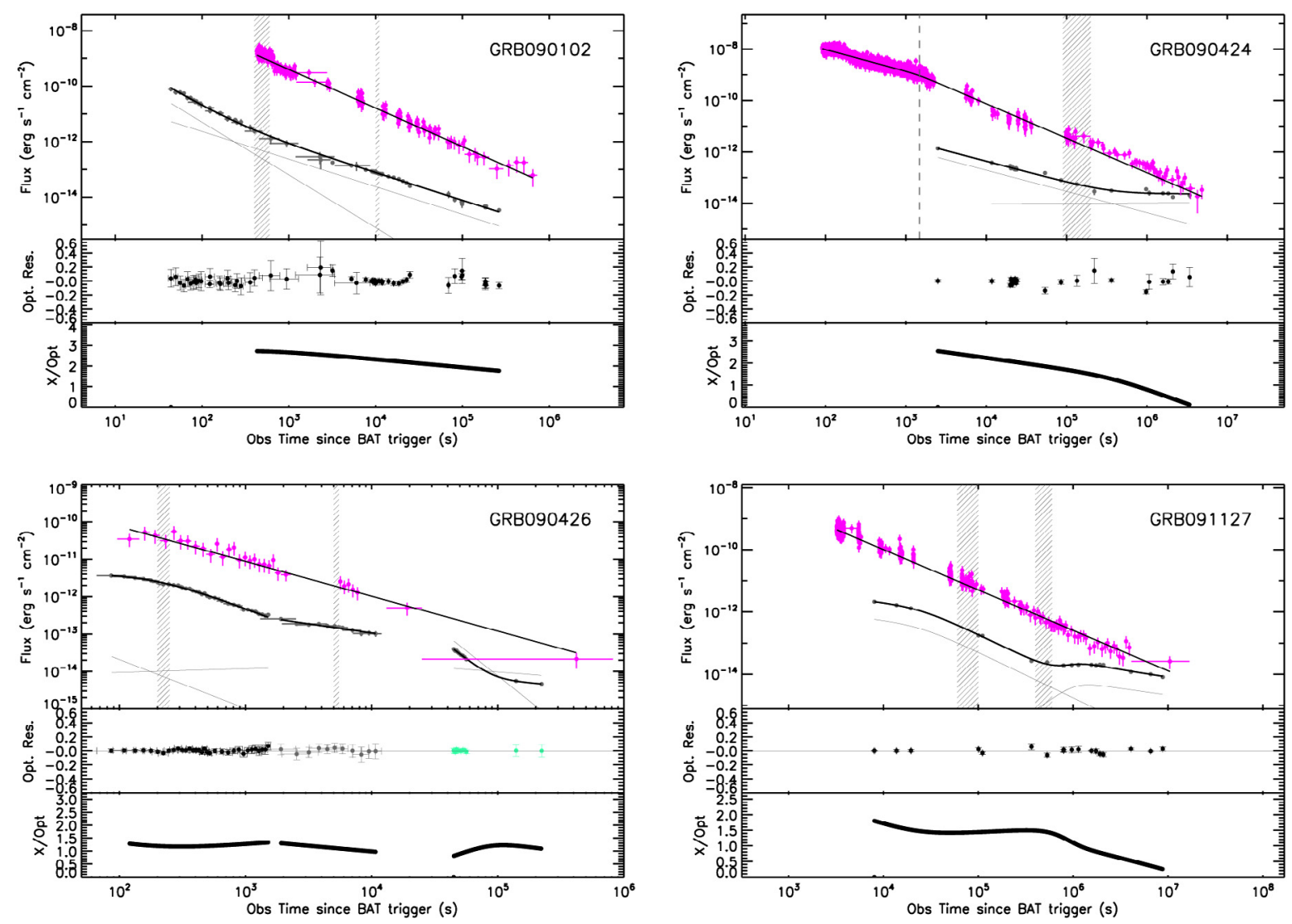

Fig. C.9. Comparison between optical and X-ray LCs. Color-coding as in Fig. C.1. 
E. Zaninoni et al.: The gamma-ray burst optical light-curve zoo: comparison with the X-ray observations
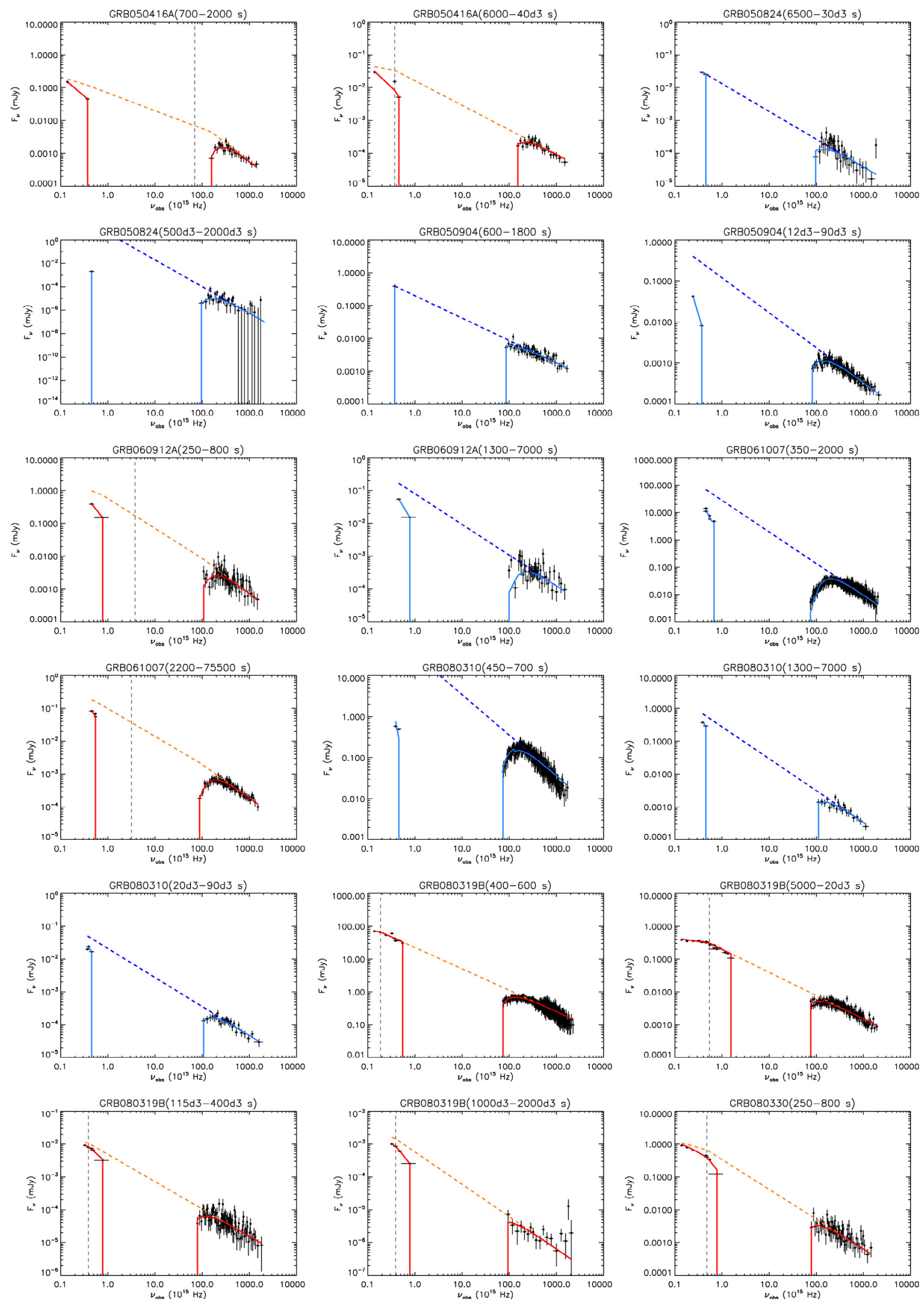

Fig. C.10. Optical/X-ray SEDs for GRBs belonging to Group A. Solid line: the fitting function. Dotted line: power-law (blue) or broken power-law (orange) fitting function. Light blue/blue lines stand for th power-law fitting functions. Red/orange lines correspond to the fitting function with the broken power-law. The distinction between the two different laws follows Table C.8. 
A\&A 557, A12 (2013)
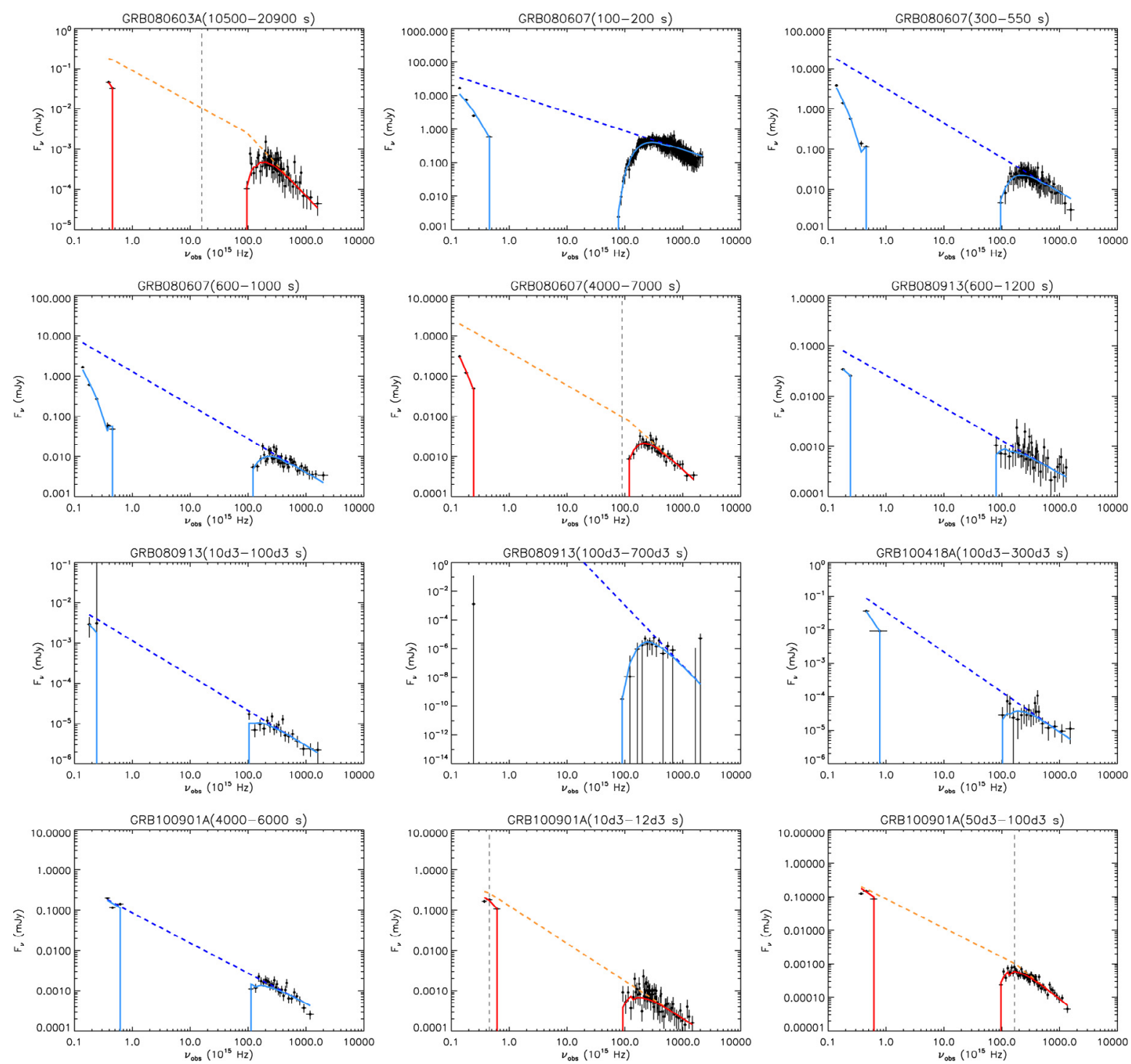

Fig. C.11. Optical/X-ray SEDs for GRBs belonging to Group A. Color-coding as in Fig. C.10. 
E. Zaninoni et al.: The gamma-ray burst optical light-curve zoo: comparison with the X-ray observations
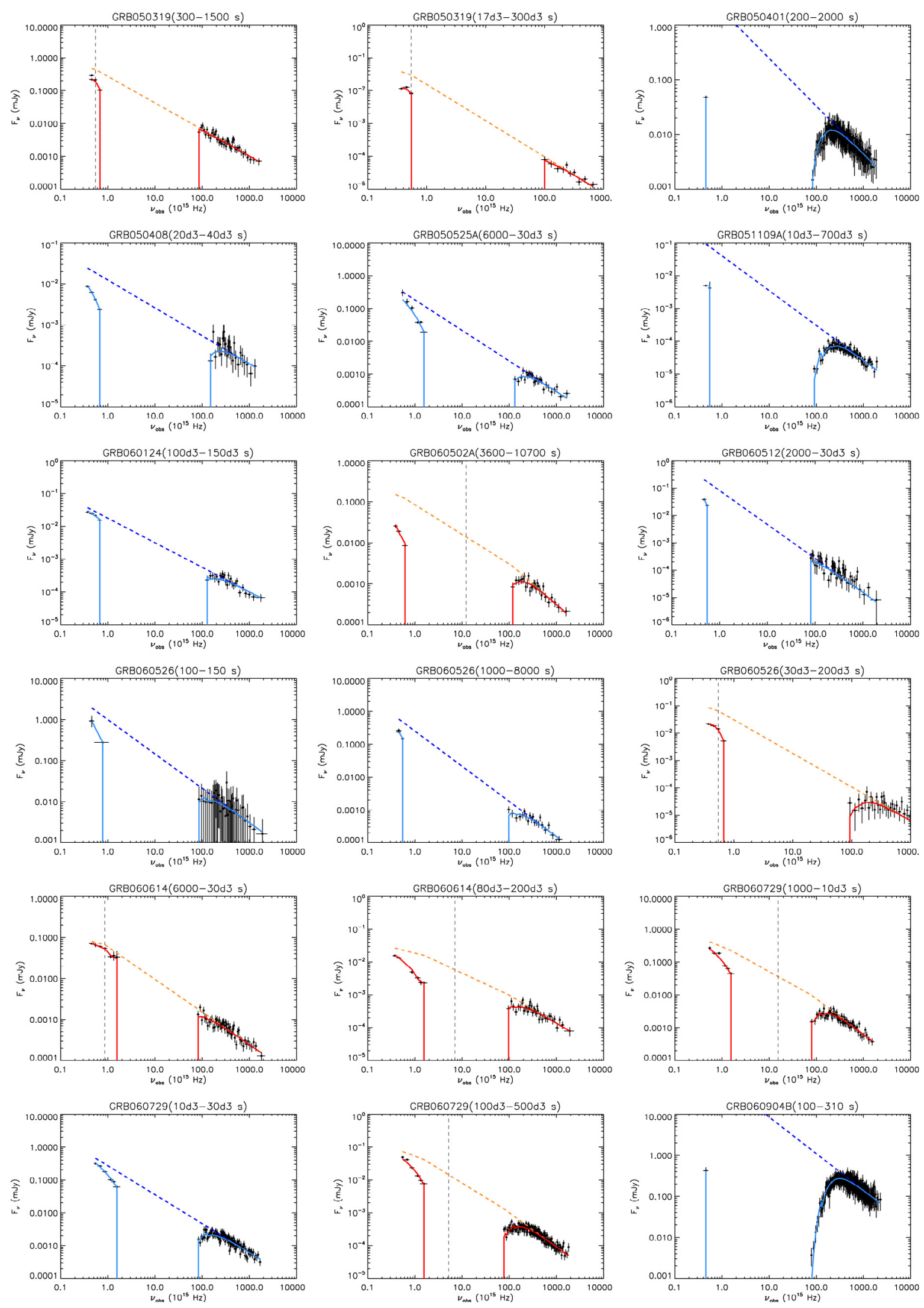

Fig. C.12. Optical/X-ray SEDs for GRBs belonging to Group B. Color-coding as in Fig. C.10. 
A\&A 557, A12 (2013)
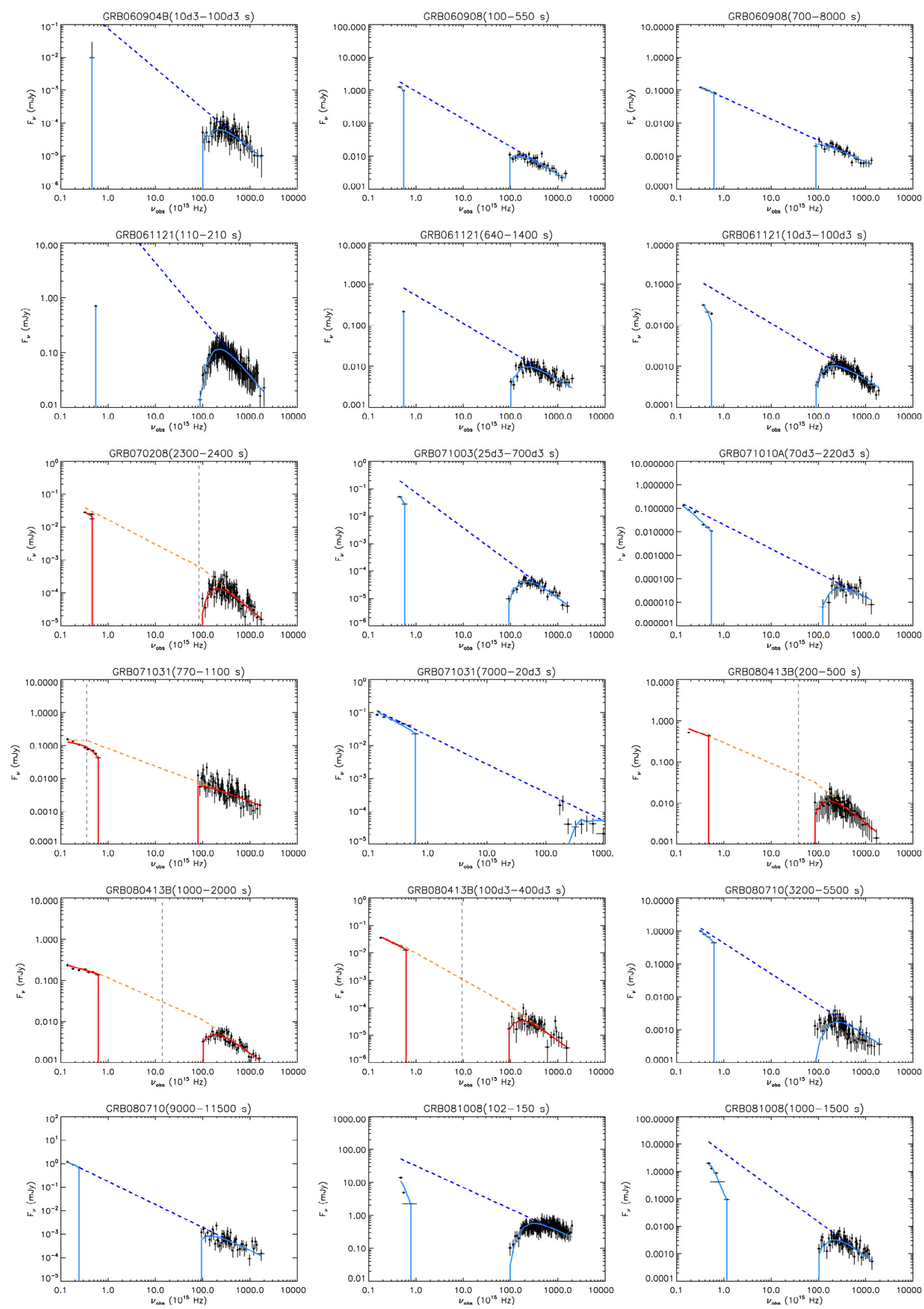

Fig. C.13. Optical/X-ray SEDs for GRBs belonging to Group B. Color-coding as in Fig. C.10. 
E. Zaninoni et al.: The gamma-ray burst optical light-curve zoo: comparison with the X-ray observations
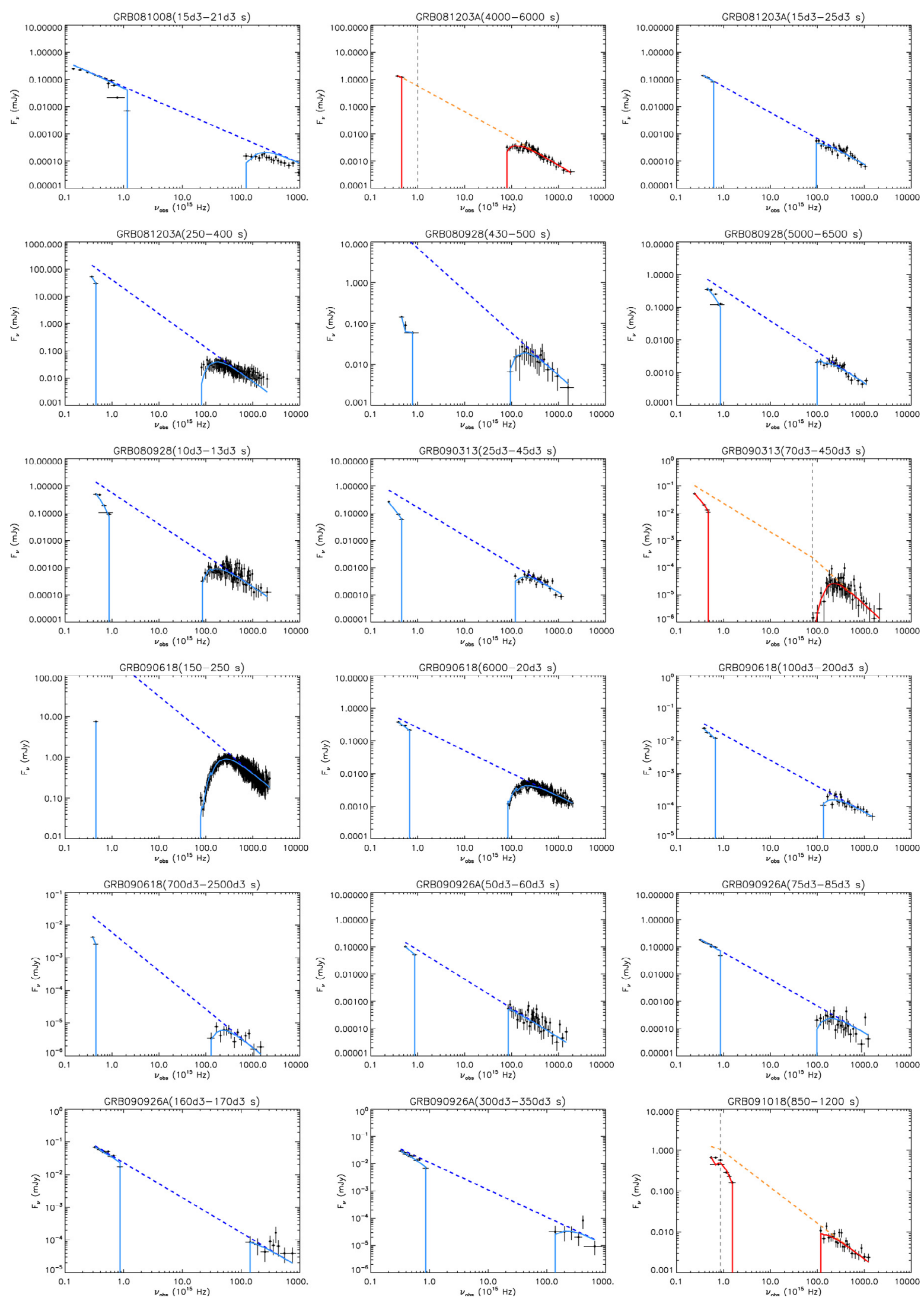

Fig. C.14. Optical/X-ray SEDs for GRBs belonging to Group B. Color-coding as in Fig. C.10. 
A\&A 557, A12 (2013)

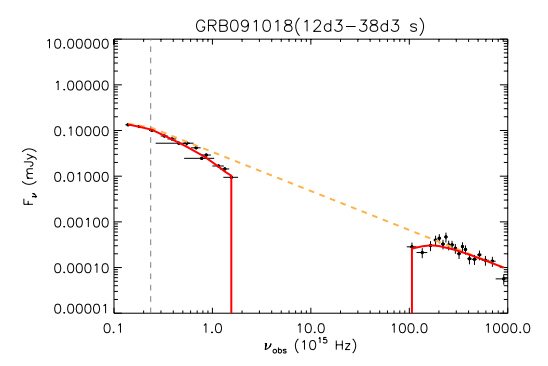

Fig. C.15. Optical/X-ray SEDs for GRBs belonging to Group B.

Color-coding as in Fig. C.10. 
E. Zaninoni et al.: The gamma-ray burst optical light-curve zoo: comparison with the X-ray observations
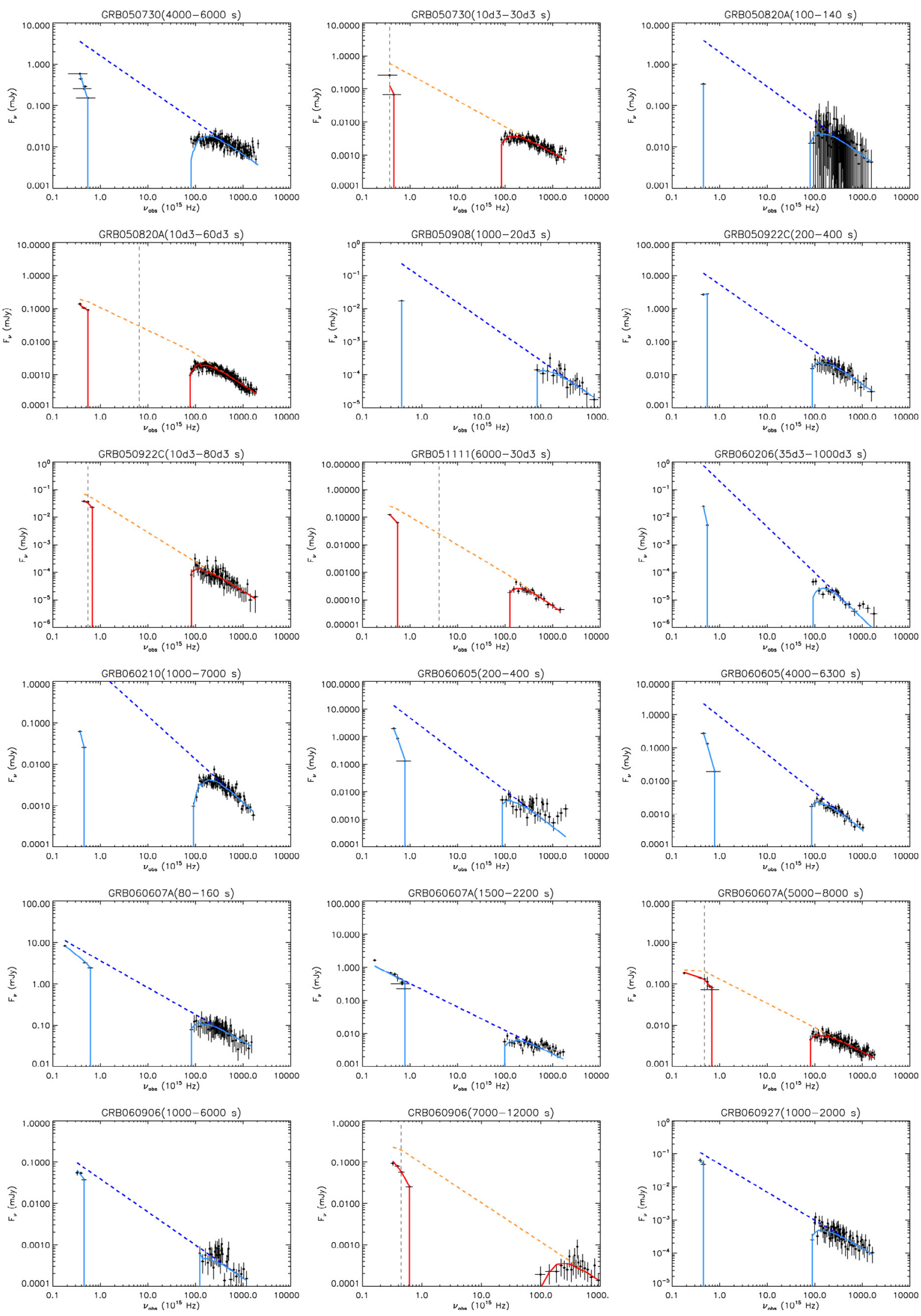

Fig. C.16. Optical/X-ray SEDs for GRBs belonging to Group C. Color-coding as in Fig. C.10. 
A\&A 557, A12 (2013)
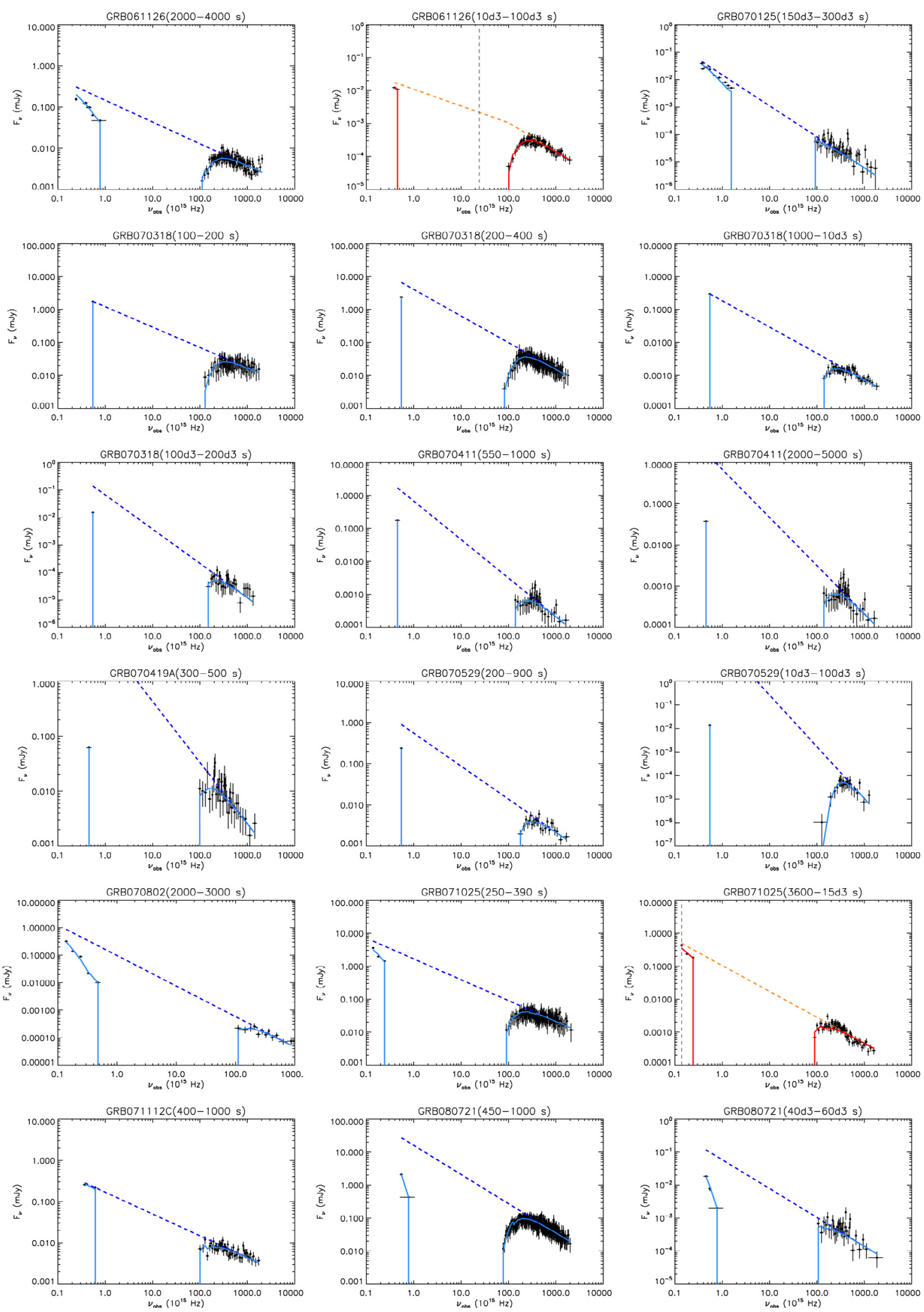

Fig. C.17. Optical/X-ray SEDs for GRBs belonging to Group C. Color-coding as in Fig. C.10. 
E. Zaninoni et al.: The gamma-ray burst optical light-curve zoo: comparison with the X-ray observations
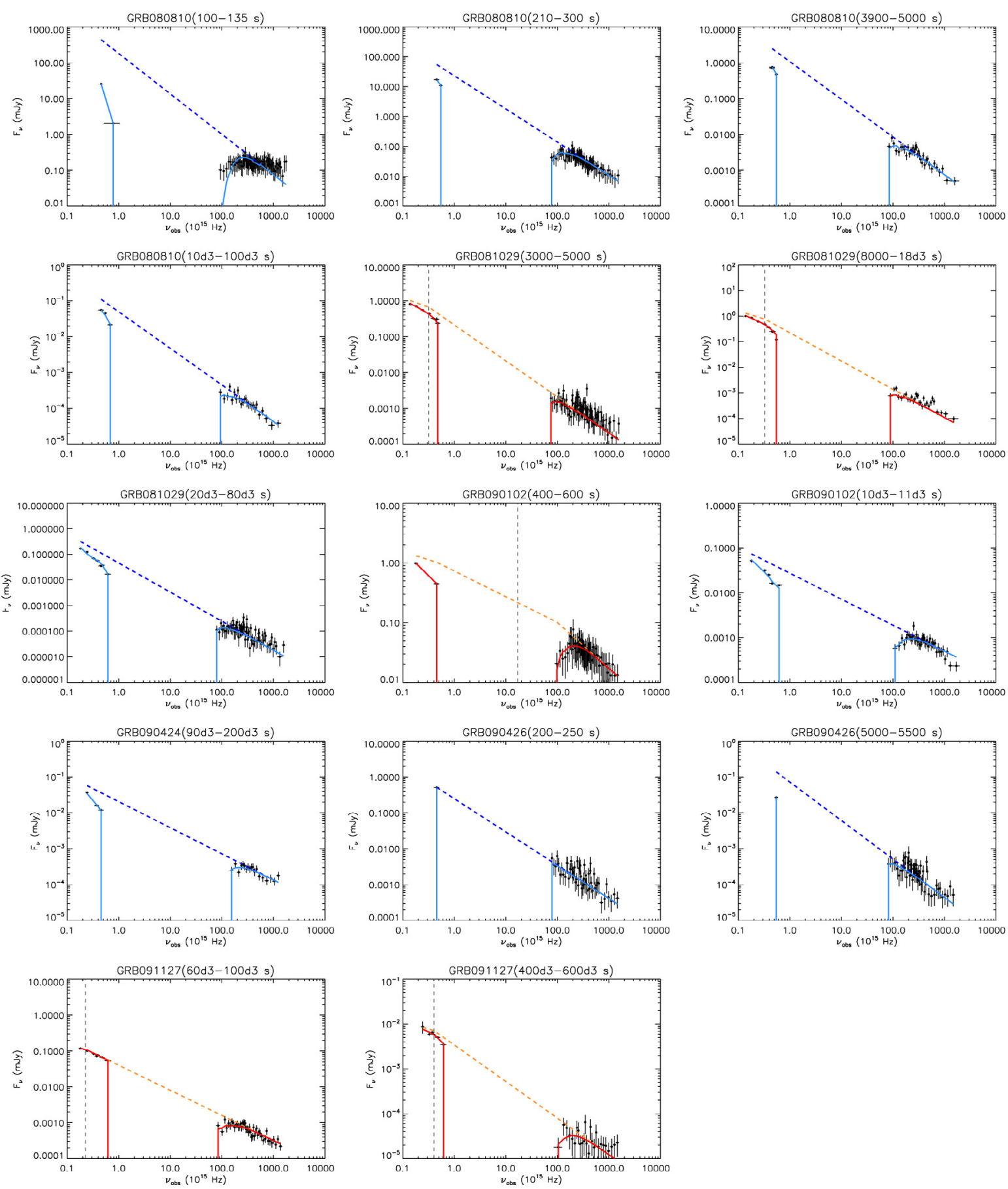

Fig. C.18. Optical/X-ray SEDs for GRBs belonging to Group C. Color-coding as in Fig. C.10. 